Medidas de assimetria bivariada e dependência local

FLÁVIO HENN FERREIRA

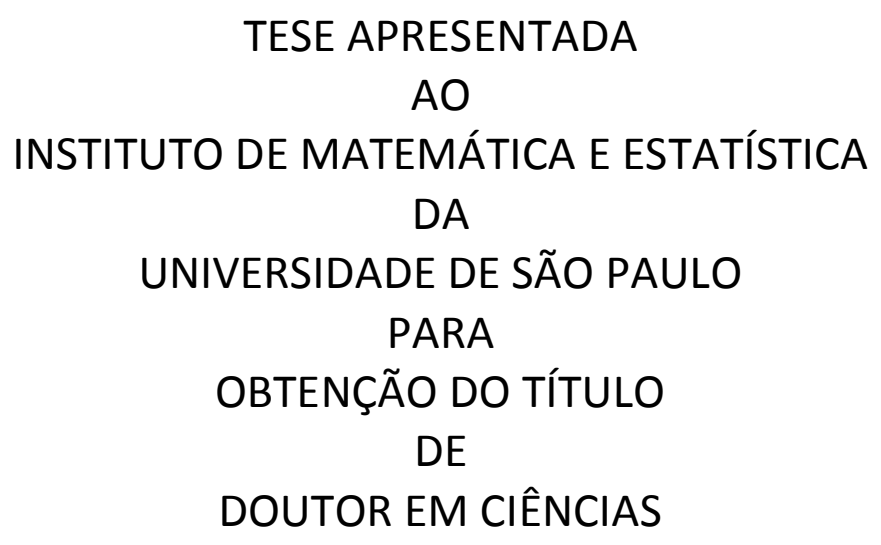

Programa: Estatística

Orientador: Prof. Dr. Nikolai V. Kolev

Co-Orientador: Prof. Dr. Roger B. Nelsen

Durante o desenvolvimento deste trabalho o autor recebeu apoio do CNPq

São Paulo, Outubro de 2008 


\section{Medidas de assimetria bivariada e dependência local}

Este exemplar corresponde à redação final da tese devidamente corrigida e defendida por Flávio Henn Ferreira e aprovada pela comissão julgadora.

\section{Banca Examinadora}

- $\quad$ Prof. Dr. Nikolai Valtchev Kolev (orientador) - IME - USP.

- Prof. Dr. Vladimir Belitsky - IME - USP.

- Profa. Dra. Beatriz Vaz de Melo Mendes - UFRJ.

- $\quad$ Prof. Dr. Cristiano Augusto Coelho Fernandes - PUC-RJ.

- Prof. Dr. Narayanaswamy Balakrishnan - UC. 


\section{Agradecimentos}

Os meus agradecimentos vão para todos aqueles que de alguma forma contribuíram para que o meu doutorado saísse da esfera do sonho e fosse para a da realidade: meu sempre paciente orientador, Nikolai Kolev, meu amigo e consultor informal, Juan Carlos Ruilova, meus amigos Adriano, Felipe, Thales e Luiz Fernando, meus familiares, enfim, todos que me ajudaram nessa caminhada. Mas um agradecimento especial vai para a Tata, que esteve sempre ao meu lado me apoiando; e para meus pais Vera e Heber, que nunca me deixaram transformar as dificuldades em impossibilidade.

Muito obrigado! 


\section{Resumo}

Esta tese trata de dois assuntos importantes na teoria de risco: o fenômeno da dependência local e a identificação e mensuração de assimetrias apresentadas pelos dados.

A primeira parte trata de dependência local, sendo abordadas algumas medidas já analisadas na literatura. Versões locais dos coeficientes de Kendall $\tau$ e Spearman $\rho$, baseadas na distribuição condicional dos dados, são propostas. São apresentadas algumas propriedades dessas medidas e a aplicação das mesmas a algumas cópulas.

Na segunda parte são apresentados resultados sobre cópulas bivariadas que são as menos associativas e menos bi-simétricas segundo o critério de máxima distância modular.

A última parte trata da não-permutabilidade e assimetria radial dos dados. Uma medida de não-permutabilidade baseada nos coeficientes de correlação condicional é proposta e aplicada a algumas distribuições. No final, o conceito de quantil bivariado é aplicado nas definições de medidas para avaliar o grau de permutabilidade e de simetria radial presentes na estrutura de dependência dos dados e de testes de hipóteses para verificar se a cópula subjacente aos dados é permutável ou radialmente simétrica.

Palavras-chave: associatividade, bi-simetria, coeficiente de correlação condicional, dependência local, permutabilidade, quantil bivariado, simetria radial. 


\begin{abstract}
In this thesis two important fields in risk theory are studied: the local dependence phenomenon and the identification and measuring of asymmetries contained in data.

The first part deals with local dependence: some measures already studied in the literature are presented and discussed, and local versions of the coefficients Kendall $\tau$ and Spearman $\rho$, based on the conditional distribution of data, are proposed. Properties of these measures and some examples concerning its application are treated.

In the second part are presented some results about bivariate copulas which are the least associative and the least bi-symmetric according to the maximum modular distance.

The last part analyses the nonexchangeability and the radial asymmetry of data. A measure of nonexchangeability based on the conditional correlation coefficient is proposed and applied to some distribution functions. At the end, the concept of bivariate quantile is applied in the definitions of measures for evaluating the degree of exchangeability and radial symmetry present in data and of hypothesis tests proposed for verifying whether the underlying copula is exchangeable or radially symmetric.
\end{abstract}

Keywords: associativity, bi-symmetry, bivariate quantile, conditional correlation coefficient, exchangeability, local dependence, radial symmetry. 


\section{Sumário}

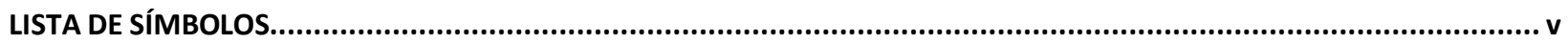

LISTA DE FIGURAS

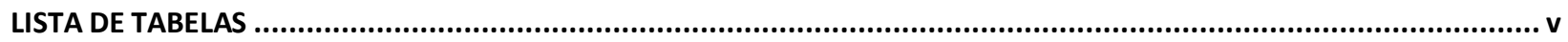

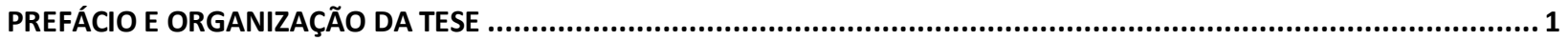

CAPÍTULO 1. FUNDAMENTOS BÁSICOS DE DEPENDÊNCIA E TEORIA DE CÓPULAS...............................................

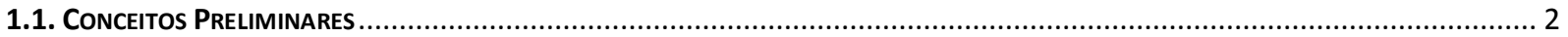

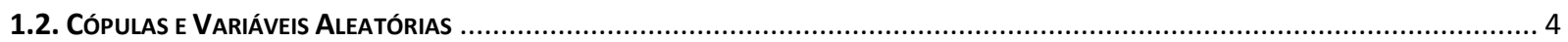

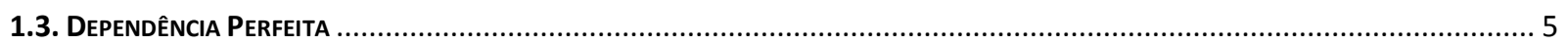

1.4. Medidas Globais de Dependência BaSeadas no Conceito de ConcordânCIA ............................................................ 6

CAPÍTULO 2. CONCEITOS E MEDIDAS DE DEPENDÊNCIA LOCAL ............................................................

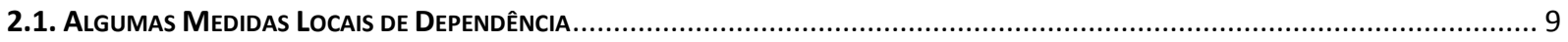

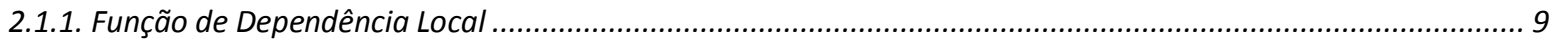

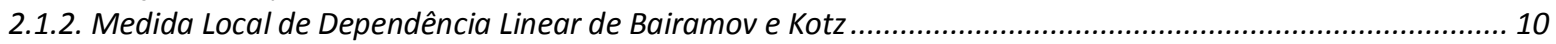

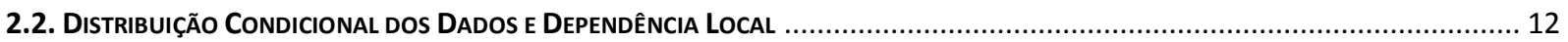

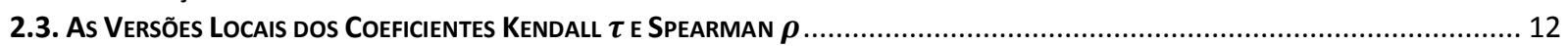

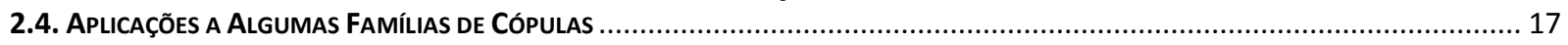

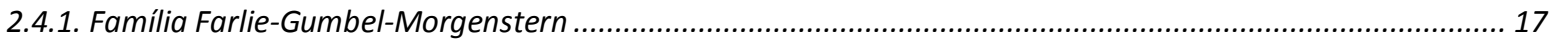

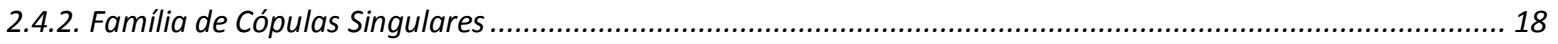

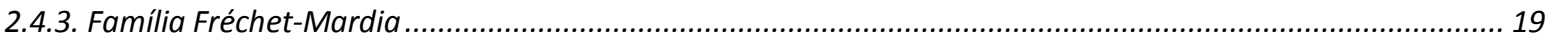

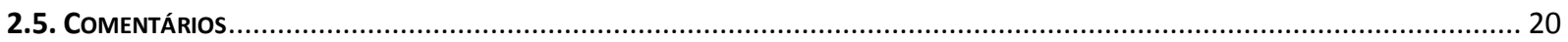

CAPÍTULO 3. ESTRUTURAS ESPECIAIS DE DEPENDÊNCIA E LIMITES MAXIMAIS DE ASSIMETRIA ...............................21

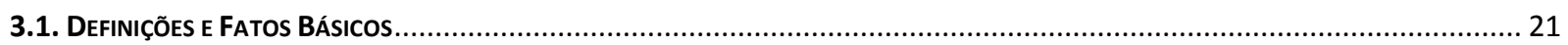

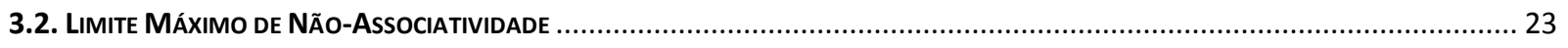

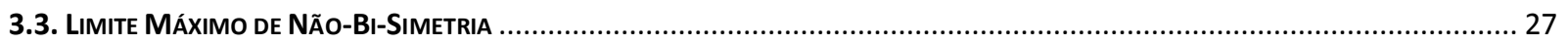

CAPÍTULO 4. MEDIDAS E TESTES DE HIPÓTESES DE ASSIMETRIA DE DISTRIBUIÇÕES BIVARIADAS............................. 33

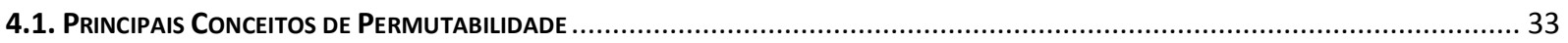

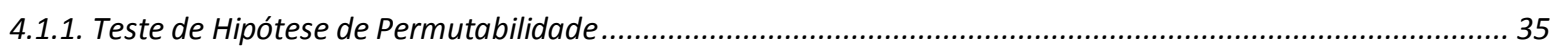

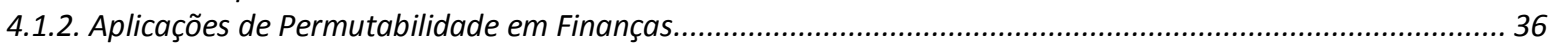

4.2. Medida de Assimetria Bivariada Baseada nos Coeficientes de Correlação Condicional ............................................ 38

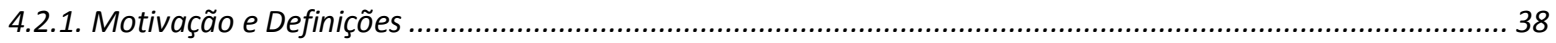

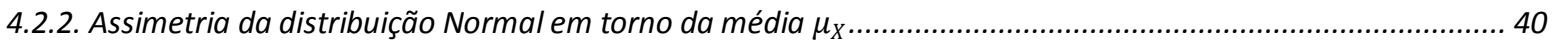

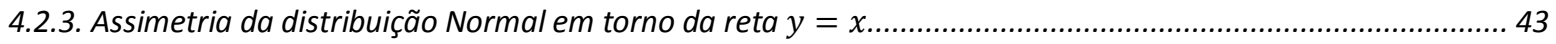

4.2.4. Assimetria da distribuição Normal Assimétrica em torno da reta $y=x$...............................................45

4.3. O Conceito de Quantil Bivariado aplicado à IdentificaÇão de Não-Permutabilidade e Assimetria Radial ................... 48

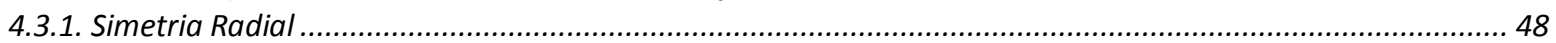

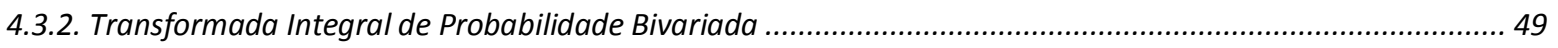

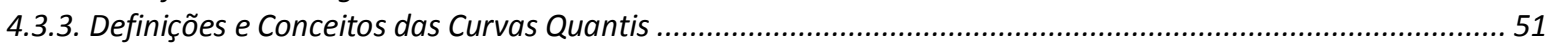

4.3.4. Aplicações dos Quantis Bivariados à Mensuração de Não-Permutabilidade e Assimetria Radial................... 54

4.3.5. Versões Empíricas e Testes de Hipóteses.............................................................................................59

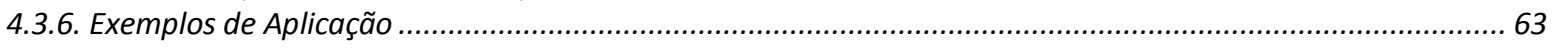

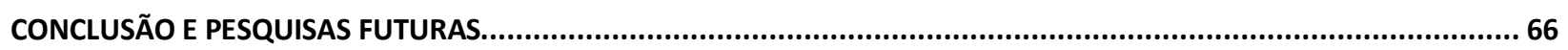

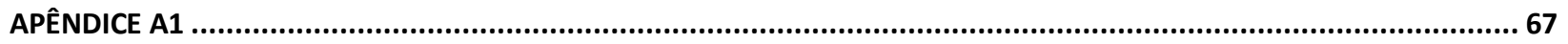

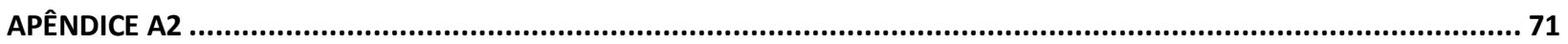

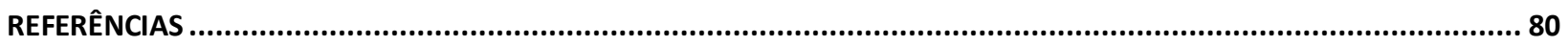




\section{Lista de Símbolos}

$\mathbb{1}(A)$ : função indicador do evento $A$

II: intervalo $[0,1]$

$\mathcal{N}(\boldsymbol{\mu}, \boldsymbol{\Sigma})$ : distribuição normal com vetor de médias $\boldsymbol{\mu}$ e matriz de covariâncias $\boldsymbol{\Sigma}$.

$\mathcal{S} \mathcal{N}(\omega, \alpha, \beta)$ : distribuição normal assimétrica bivariada com parâmetro $\omega$ de correlação e parâmetros $\alpha, \beta$ de assimetria

$\mathfrak{R}$ : reta real $(-\infty, \infty)$

$\overline{\mathfrak{R}}$ : reta real estendida $[-\infty, \infty]$

$P($. ): função probabilidade

$E($. ): função esperança

$E(. \mid A)$ : função esperança condicional ao evento $A$

$\operatorname{Var}($.$) : função variância$

$\operatorname{Var}(. \mid A)$ : função variância condicional ao evento $A$

$\operatorname{Cov}(X, Y)$ : função covariância entre as variáveis aleatórias $X$ e $Y$

$\rho_{X, Y}, \operatorname{Corr}(X, Y)$ : Coeficiente de correlação de Pearson entre as variáveis aleatórias $X$ e $Y$

$U(a, b)$ : distribuição uniforme contínua no intervalo $(a, b)$

: distribuído como

$\operatorname{Max}($.): função máximo

$\operatorname{Min}($.$) : função mínimo$

Sup: supremo

Inf : ínfimo

$F^{-1}$ : função inversa de $F$

$\Omega$ : espaço amostral

\section{Lista de Figuras}

Figura 2.1. Comparação de $\tau_{L_{C}}$ e $\rho_{L_{C}}$ para a família Farlie-Gumbel-Morgenstern de cópulas.

Figura 3.1. Os suportes de $C_{1}$ e $C_{2}$.

Figura 4.1: Curvas quantis bivariadas: (a) $p \leq \frac{1}{2}$, (b) $p>\frac{1}{2}$.

Figura 4.2: A região central.

Figura 4.3: Exemplo de cópula $C(u, v)$ tal que $C(u, v) \neq C(v, u)$, mas $\operatorname{com} \Sigma_{C}=0$.

\section{Lista de Tabelas}

Tabela 4.1. Formulações de Permutabilidade.

Tabela 4.2. $\left(C_{a} \mid C_{b}\right)(t)$. 


\section{Prefácio e Organização da Tese}

Sempre foi atual o estudo do fenômeno da dependência na modelagem de dados das mais diversas naturezas. Tanto no meio acadêmico quanto na indústria de maneira geral, modelagens que deixam de levar a dependência em conta são cada vez mais questionadas e seu uso menos freqüente. Isso se deve em boa parte pela difusão do conhecimento do que é a dependência entre variáveis aleatórias, bem como das implicações que a suposição de independência acarreta. Um exemplo disso é a indústria financeira, onde a busca por distribuições que descrevam de maneira fiel os dados analisados tem recebido grande atenção, tanto por suas implicações na análise de risco de portfólios, como no apreçamento de produtos sofisticados que envolvem mais de uma variável financeira, onde a utilização de modelos mais corretos para o comportamento conjunto dos dados pode significar a diferença entre o lucro e o prejuízo. Outros exemplos de áreas onde a dependência desempenha papel relevante seriam: a indústria de seguros, institutos previdenciários, indústria médico-farmacêutica, etc.

Dentro desse contexto, a idéia desta tese é ampliar o entendimento sobre algumas formas específicas de dependência, bem como identificar formas mais completas e eficazes de mensurar a associação entre variáveis aleatórias. Então, os objetivos básicos desta tese são:

1) Propor e estudar as propriedades de duas medidas locais de dependência: as versões locais dos coeficientes Kendall $\tau$ e Spearman $\rho$;

2) Estudar distâncias máximas entre formas de dependência segundo algumas medidas definidas. As estruturas que serão tratadas são as associativas e bi-simétricas;

3) Propor e estudar medidas de não-permutabilidade e assimetria radial bivariadas, bem como os testes de hipóteses para a verificação da existência das mesmas nos dados.

No capítulo 1 são apresentados os conceitos básicos de cópulas e algumas propriedades que serão utilizadas ao longo do texto. No capítulo 2 será tratado o fenômeno da dependência local, sendo apresentadas algumas medidas locais de dependência já estudadas na literatura e propostas duas novas medidas locais de dependência. Suas propriedades serão estudadas, bem como suas aplicações para algumas famílias de cópulas. No capítulo 3 serão definidas as estruturas de dependência associativas e bi-simétricas e apresentados os resultados sobre as formas de dependências que estão mais distantes destas, segundo medidas definidas caso a caso. No capítulo 4 os fenômenos da não-permutabilidade e da assimetria radial são estudados. Uma primeira tentativa de mensurar a ausência de permutabilidade dos dados a partir do coeficiente de correlação condicional é apresentada. Aplicações dessa medida às distribuições normal e normal assimétrica são obtidas, bem como contra-exemplos interessantes e contraintuitivos. Na seqüência, medidas e testes de hipóteses são propostos para avaliação da nãopermutabilidade e da assimetria radial da estrutura de dependência dos dados, a partir do conceito de curvas quantis bivariadas. Finalmente fazemos uma conclusão a respeito dos resultados obtidos e propomos alguns tópicos a serem pesquisados futuramente. A seguir são apresentadas, em dois apêndices, as fórmulas desenvolvidas para os coeficientes de correlação condicional (dado um condicionamento específico) nos casos: normal simétrico e assimétrico. A tese termina com as referências bibliográficas utilizadas. 


\section{Capítulo 1.}

\section{Fundamentos Básicos de Dependência e Teoria de Cópulas}

Neste capítulo trataremos, seguindo Embrechts et al. (2003) e Nelsen (2006), das principais definições e dos resultados utilizados posteriormente a respeito da teoria de cópulas, dando uma atenção especial a algumas cópulas especiais, que são os chamados limites de FréchetHoeffding, e à efetiva aplicação de cópulas ao estudo de variáveis aleatórias.

Este capítulo serve como base para uma multiplicidade de aplicações dos conceitos aqui apresentados que serão feitas nos capítulos seguintes, sendo dessa forma relevante a apresentação dos conceitos aqui mostrados.

\subsection{Conceitos Preliminares}

Seja $\boldsymbol{X}=\left(X_{1}, \ldots, X_{n}\right)$ um vetor aleatório com distribuição conjunta $F_{\boldsymbol{X}}$ e distribuições marginais $F_{X_{1}}, \ldots, F_{X_{n}}$. Dizemos que os componentes de $\boldsymbol{X}$ são mutuamente independentes se e somente se, para todo $\boldsymbol{x}=\left(x_{1}, \ldots, x_{n}\right) \in \mathfrak{R}^{n}$, temos que a seguinte igualdade se verifica:

$$
F_{X}\left(x_{1}, \ldots, x_{n}\right)=\prod_{i=1}^{n} F_{X_{i}}\left(x_{i}\right)
$$

A independência entre duas variáveis aleatórias $X_{1}$ e $X_{2}$ pode ser interpretada como a propriedade de a variável $X_{1}$ não ter o seu comportamento associado ao comportamento da variável $X_{2}$, e vice-versa.

É bem conhecido que a dependência entre duas variáveis aleatórias é totalmente definida pela função de distribuição conjunta dessas variáveis, ou seja, todo o conhecimento a respeito das características da dependência entre as variáveis está encerrado nesta função. De uma maneira mais geral, todo o conhecimento a respeito das variáveis $X_{1}, \ldots, X_{n}$ e de suas inter-relações, está contido na função distribuição conjunta

$$
F_{X}\left(x_{1}, \ldots, x_{n}\right)=P\left(X_{1} \leq x_{1}, \ldots, X_{n} \leq x_{n}\right)
$$

Uma forma padronizada de extrair a estrutura de dependência das variáveis aleatórias contínuas é através do conceito de cópulas, que é definido a seguir.

Definição 1.1. Uma cópula é definida como a função de distribuição conjunta:

$$
C\left(u_{1}, \ldots, u_{n}\right)=P\left(U_{1} \leq u_{1}, \ldots, U_{n} \leq u_{n}\right), \quad 0 \leq u_{i} \leq 1, \quad i=1, \ldots, n,
$$

sendo $U_{i}$ variáveis aleatórias (v.a.'s) uniformes no intervalo $(0,1)$. 
Sejam $X_{1}, \ldots, X_{n}$ v.a.'s contínuas com distribuições marginais $F_{X_{1}}, \ldots, F_{X_{n}}$, respectivamente. A partir da Definição 1.1 temos a seguinte relação:

$$
F_{X}\left(x_{1}, \ldots, x_{n}\right)=C\left(F_{X_{1}}\left(x_{1}\right), \ldots, F_{X_{n}}\left(x_{n}\right)\right)
$$

O teorema de Sklar, veja Nelsen (2006), dá a existência e unicidade da função $C($.$) em$ (1.1) se as marginais $F_{X_{1}}, \ldots, F_{X_{n}}$ são contínuas. Se algumas das marginais $F_{X_{1}}, \ldots, F_{X_{n}}$ são discretas, a relação (1.1) é válida, mas $C$ (.) não é mais única. As transformações não lineares estritamente crescentes alteram a distribuição conjunta $F_{\boldsymbol{X}}($.$) e as suas marginais, mas a$ forma analítica da cópula $C($. ) permanece a mesma. Esta é uma característica importante e desejável.

Exemplo. Seja $\Phi$ a função distribuição normal padrão univariada e seja $\Phi_{\Sigma}^{n}$ a função distribuição normal padrão multivariada com matriz de correlações $\Sigma$. Então temos que

$$
C\left(u_{1}, \ldots, u_{n}\right)=\Phi_{\Sigma}^{\mathrm{n}}\left(\Phi^{-1}\left(x_{1}\right), \ldots, \Phi^{-1}\left(x_{\mathrm{n}}\right)\right)
$$

é a cópula normal $n$-variada, onde $\Phi^{-1}($. ) é a função inversa de $\Phi$.

Nós denotamos por $\bar{F}_{\boldsymbol{X}}$ a função de sobrevivência conjunta para $n$ variáveis aleatórias com função distribuição conjunta $F_{X}$, isto é, se $\left(X_{1}, \ldots, X_{n}\right)$ tem função distribuição conjunta $F_{\boldsymbol{X}}$, então $\bar{F}_{\boldsymbol{X}}\left(x_{1}, \ldots, x_{n}\right)=P\left(X_{1}>x_{1}, \ldots, X_{n}>x_{n}\right)$. Analogamente $\bar{C}$ representa a função conjunta de sobrevivência de $U_{i}$ v.a.'s uniformes $U(0,1)$ e $0 \leq u_{i} \leq 1, i=1, \ldots, n$, e que tem a cópula $C$ como função distribuição, isto é,

$$
\bar{C}\left(u_{1}, \ldots, u_{n}\right)=P\left(U_{1}>u_{1}, \ldots, U_{n}>u_{n}\right)
$$

A cópula de sobrevivência $\hat{C}(u, v)$ associada à cópula $C(u, v)$ é definida como a cópula do vetor $(1-U, 1-V)$, onde $(U, V)$ é um vetor de variáveis aleatórias uniformemente distribuídas em $(0,1)$, e que tem cópula $C(u, v)$. Dessa forma temos que

$$
\hat{C}(u, v)=u+v-1+C(1-u, 1-v)
$$

Definição 1.2. Se $C_{1}$ e $C_{2}$ são cópulas, $C_{1}$ é menor que $C_{2}$ (denotado por $C_{1} \prec C_{2}$ ) se

$$
C_{1}(\boldsymbol{u}) \leq C_{2}(\boldsymbol{u}) \text { e } \bar{C}_{1}(\boldsymbol{u}) \leq \bar{C}_{2}(\boldsymbol{u}) \text {, para todo } \boldsymbol{u} \in[0,1]^{n}
$$

Note que no caso bivariado

$$
\begin{aligned}
\bar{C}_{1}\left(u_{1}, u_{2}\right) \leq \bar{C}_{2}\left(u_{1}, u_{2}\right) & \Leftrightarrow 1-u_{1}-u_{2}+\bar{C}_{1}\left(u_{1}, u_{2}\right) \leq 1-u_{1}-u_{2}+\bar{C}_{1}\left(u_{1}, u_{2}\right) \\
& \Leftrightarrow C_{1}\left(u_{1}, u_{2}\right) \leq C_{2}\left(u_{1}, u_{2}\right)
\end{aligned}
$$




\subsection{Cópulas e Variáveis Aleatórias}

Sejam $X_{1}, \ldots, X_{n}$ variáveis aleatórias com funções distribuição contínuas $F_{X_{1}}, \ldots, F_{X_{n}}$, respectivamente, e função distribuição conjunta $F_{X}$. Então $\left(X_{1}, \ldots, X_{n}\right)$ tem uma única cópula $C$, onde $C$ é dada por (1.1).

A representação padrão em termos de cópula da distribuição do vetor aleatório $\left(X_{1}, \ldots, X_{n}\right)$ é então:

$$
F_{X}\left(x_{1}, \ldots, x_{n}\right)=P\left(X_{1} \leq x_{1}, \ldots, X_{n} \leq x_{n}\right)=C\left(F_{X_{1}}\left(x_{1}\right), \ldots, F_{X_{n}}\left(x_{n}\right)\right)
$$

As transformações $X_{i} \mapsto F_{i}\left(X_{i}\right), i=1, \ldots, n$, usadas na representação acima formam uma ferramenta padrão na metodologia de simulação, denominada como Transformada Integral de Probabilidade.

Uma vez que $X_{1}, \ldots, X_{n}$ são independentes se e somente se para todos $x_{1}, \ldots, x_{n}$ em $\bar{\Re}$,

$$
F_{X}\left(x_{1}, \ldots, x_{n}\right)=\prod_{i=1}^{n} F_{X_{i}}\left(x_{i}\right) \text {, }
$$

o próximo resultado segue da expressão (1.1) e do teorema de Sklar, veja Nelsen (2006).

Teorema 1.1. [Nelsen (2006)] Seja $\left(X_{1}, \ldots, X_{n}\right)$ um vetor de variáveis aleatórias contínuas com cópula $C$, então $X_{1}, \ldots, X_{n}$ são independentes se e somente se $C(\boldsymbol{u})=\prod_{i=1}^{n} u_{i}, \boldsymbol{u} \in[0,1]^{n}$.

Uma propriedade conveniente das cópulas é que para transformações estritamente monótonas das variáveis aleatórias, as cópulas das varáveis transformadas são invariantes, ou mudam de maneira simples. Note que se a função distribuição da variável aleatória $Y$ é contínua, e se $\alpha$ é uma função estritamente monótona cujo domínio contém a imagem de $Y$, então a função distribuição da variável aleatória $\alpha(Y)$ é também contínua.

Teorema 1.2. [Embrechts et al. (2003)] Seja $\left(X_{1}, \ldots, X_{n}\right)$ um vetor de variáveis aleatórias contínuas com cópula $C_{X_{1}, \ldots, X_{n}}$. Se $\alpha_{1}, \ldots, \alpha_{n}$ são estritamente monótonos em $\operatorname{Im}\left(X_{1}\right), \ldots, \operatorname{Im}\left(X_{n}\right)$, respectivamente, e seja $\left(\alpha_{1}\left(X_{1}\right), \ldots, \alpha_{n}\left(X_{n}\right)\right)$ um vetor de variáveis

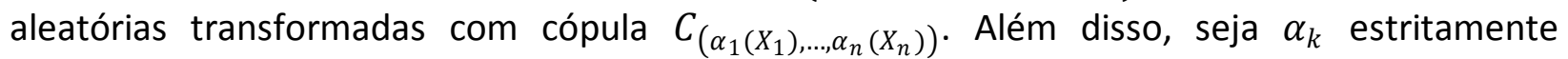
decrescente para algum $k$. Sem perda de generalidade, seja $k=1$. Então temos que

$$
C_{\alpha_{1}\left(X_{1}\right), \ldots, \alpha_{n}\left(X_{n}\right)}\left(u_{1}, \ldots, u_{n}\right)=C_{\alpha_{2}\left(X_{2}\right), \ldots, \alpha_{n}\left(X_{n}\right)}\left(u_{2}, \ldots, u_{n}\right)-C_{X_{1}, \alpha_{2}\left(X_{2}\right), \ldots, \alpha_{n}\left(X_{n}\right)}\left(1-u_{1}, u_{2}, \ldots, u_{n}\right)
$$




\subsection{Dependência Perfeita}

Os Limites de Fréchet-Hoeffding, $M^{n}$ e $W^{n}$, e a cópula de independência, $\Pi^{n}$, para funções distribuição conjuntas são definidos através das funções $M^{n}, \Pi^{n}, W^{n}$ definidas em $[0,1]^{n}$ como a seguir:

$$
\begin{aligned}
& M^{\boldsymbol{n}}(\boldsymbol{u})=\operatorname{Min}\left(u_{1}, \ldots, u_{n}\right) \\
& \Pi^{\boldsymbol{n}}(\boldsymbol{u})=\prod_{i=1}^{n} u_{i} \\
& W^{\boldsymbol{n}}(\boldsymbol{u})=\operatorname{Max}\left(u_{1}+\ldots+u_{n}-(n-1), 0\right) .
\end{aligned}
$$

As funções $M^{n}$ e $\Pi^{n}$ são cópulas $n$-dimensionais para todos $n \geq 2$, ao passo que a função $W^{n}$ não é uma cópula para todos $n \geq 3$.

O próximo teorema é denominado desigualdade de Fréchet - Hoeffding.

Teorema 1.3. [Nelsen (2006)] Se $C$ é uma $n$-cópula qualquer, então para todo $\boldsymbol{u} \in[0,1]^{n}$,

$$
W^{n}(\boldsymbol{u}) \leq C(\boldsymbol{u}) \leq M^{n}(\boldsymbol{u})
$$

Para maiores detalhes, incluindo interpretações geométricas, ver Mikusinski et al. (1992). Embora o limite inferior de Fréchet-Hoeffding $W^{n}$ não seja sempre uma cópula, ele é o melhor limite inferior possível, da seguinte maneira.

Teorema 1.4. [Nelsen (2006)] Para qualquer $n \geq 3$ e qualquer $\boldsymbol{u} \in[0,1]^{n}$, existe uma cópula $C$ (que depende de $\boldsymbol{u}$ ) tal que $C(\boldsymbol{u})=W^{n}(\boldsymbol{u})$.

No caso bidimensional, os limites superior, $M$, e inferior, $W$, são cópulas e $W$ e $M$ são as funções distribuições conjuntas bivariadas dos vetores aleatórios $(U, 1-U)$ e $(U, U)$ respectivamente, onde $U$ é uma variável aleatória com distribuição uniforme em $(0,1)$. Neste caso, $W$ e $M$ descrevem as dependências negativa perfeita e positiva perfeita, respectivamente.

Teorema 1.5. [Embrechts et al. (2003)] Seja $(X, Y)$ com cópula $W=\operatorname{Max}(u+v-1,0)$ ou $M=\operatorname{Min}(u, v)$. Então existem duas funções monótonas $\alpha, \beta: \mathfrak{R} \rightarrow \mathfrak{R}$ e uma variável aleatória $Z$ tal que

$$
(X, Y) \stackrel{d}{=}(\alpha(Z), \beta(Z))
$$

onde $\stackrel{d}{=}$ indica igualdade em distribuição, com $\alpha$ crescente e $\beta$ decrescente (ou vice-versa) no caso de $(X, Y)$ ter cópula $W$; e com ambos $\alpha$ e $\beta$ crescentes, ou decrescentes, no caso de $(X, Y)$ ter cópula $M$. A volta do teorema também é válida. 
É importante notar que se qualquer uma das distribuições marginais, seja de $X$ ou de $Y$, tiver descontinuidades, a cópula de $X$ e $Y$ não é única, sendo $M$ ou $W$ cópulas possíveis.

No caso onde as marginais são contínuas, uma versão mais forte do teorema pode ser apresentada:

$C=W$ se e somente se $Y=T(X)$ quase certamente, $T=G^{-1} \circ(1-F)$ decrescente;

$C=W$ se e somente se $Y=T(X)$ quase certamente, $T=G^{-1} \circ(F)$ crescente,

(o símbolo o indica função composta).

\subsection{Medidas Globais de Dependência Baseadas no Conceito de Concordância}

Nesta seção trataremos do conceito de concordância e a sua aplicação na definição das medidas de dependência Kendall $\tau$ e Spearman $\rho$.

Sejam $(x, y)$ e $\left(x^{\prime}, y^{\prime}\right)$ duas observações de um vetor $(X, Y)$ de variáveis aleatórias contínuas. Então $(x, y)$ e $\left(x^{\prime}, y^{\prime}\right)$ são ditos concordantes se $\left(x-x^{\prime}\right)\left(y-y^{\prime}\right)>0$ e discordantes se $\left(x-x^{\prime}\right)\left(y-y^{\prime}\right)<0$.

Teorema 1.6. [Nelsen (2006)] Sejam $(X, Y)$ e $\left(X^{\prime}, Y^{\prime}\right)$ vetores independentes de variáveis aleatórias contínuas, com funções distribuição conjuntas $H$ e $H^{\prime}$, respectivamente, com marginais comuns $F$ (de $X$ e $X^{\prime}$ ) e $G$ (de $Y$ e $Y^{\prime}$ ). Sejam $C$ e $C^{\prime}$ as cópulas de $(X, Y)$ e $\left(X^{\prime}, Y^{\prime}\right)$ respectivamente de modo que $H(x, y)=C(F(x), G(y))$ e $H^{\prime}(x, y)=C^{\prime}(F(x), G(y))$. Seja $Q$ a diferença entre a probabilidade de concordância e a de discordância de $(X, Y)$ e $\left(X^{\prime}, Y^{\prime}\right)$, isto é, seja

$$
Q=P\left(\left(X-X^{\prime}\right)\left(Y-Y^{\prime}\right)>0\right)-P\left(\left(X-X^{\prime}\right)\left(Y-Y^{\prime}\right)<0\right) .
$$

Então

$$
Q=Q\left(C, C^{\prime}\right)=4 \iint_{[0,1]^{2}} C^{\prime}(u, v) d C(u, v)-1
$$

Corolário. [Nelsen (2006)] Sejam $C, C^{\prime}$ e $Q$ como definidos no teorema anterior, então

(i) $Q$ é simétrica em seus argumentos: $Q\left(C, C^{\prime}\right)=Q\left(C^{\prime}, C\right)$;

(ii) $Q$ é não-decrescente em cada argumento: se $C_{1} \prec C_{1}{ }^{\prime}$ e $C_{2} \prec C_{2}{ }^{\prime}$ para todos $(u, v) \in[0,1]^{2}$, então $Q\left(C_{1}, C_{2}\right) \leq Q\left(C_{1}^{\prime}, C_{2}^{\prime}\right)$;

(iii) As cópulas $C$ e $C^{\prime}$ podem ser substituídas pelas respectivas cópulas de sobrevivência $\hat{C}$ e $\hat{C}^{\prime}$ em $Q$, isto é, $Q\left(C, C^{\prime}\right)=Q\left(\hat{C}, \hat{C}^{\prime}\right)$. 
A seguir discutimos duas importantes medidas de dependência (concordância) conhecidas como Kendall $\tau$ e Spearman $\rho$. As duas são talvez as melhores alternativas em relação ao coeficiente de correlação linear como medidas de dependência para distribuições não-elípticas.

Definição 1.3. A medida Kendall $\tau$ para um vetor $(X, Y)$ é definida como:

$$
\tau(X, Y)=P\left(\left(X-X^{\prime}\right)\left(Y-Y^{\prime}\right)>0\right)-P\left(\left(X-X^{\prime}\right)\left(Y-Y^{\prime}\right)<0\right)
$$

onde $\left(X^{\prime}, Y^{\prime}\right)$ é uma cópia independente de $(X, Y)$.

Portanto, o Kendall $\tau$ para $(X, Y)$ é simplesmente a medida $Q$ com $C=C^{\prime}$, definida na seção anterior, ou seja, é a diferença entre as probabilidades de concordância e discordância.

Teorema 1.7. [Nelsen (2006)] Seja $(X, Y)$ um vetor de variáveis aleatórias contínuas com cópula $C$. Então o Kendall $\tau$ para $(X, Y)$ é dado por

$$
\tau(X, Y)=Q(C, C)=4 \iint_{[0,1]^{2}} C(u, v) d C(u, v)-1
$$

É importante notar que a integral acima é o valor esperado da variável aleatória $C(U, V)$, onde $U, V \sim U(0,1)$ com função distribuição conjunta $C$, isto é,

$$
\tau(X, Y)=4 E(C(U, V))-1
$$

Definição 1.4. A medida Spearman $\rho$ para o vetor aleatório $(X, Y)$ é definida como:

$$
\rho(X, Y)=3\left[P\left(\left(X-X^{\prime}\right)\left(Y-Y^{\prime \prime}\right)>0\right)-P\left(\left(X-X^{\prime}\right)\left(Y-Y^{\prime \prime}\right)<0\right)\right]
$$

onde $(X, Y),\left(X^{\prime}, Y^{\prime}\right)$ e $\left(X^{\prime \prime}, Y^{\prime \prime}\right)$ são cópias independentes.

Teorema 1.8. [Nelsen (2006)] Seja $(X, Y)$ um vetor aleatório de variáveis aleatórias contínuas com cópula $C$. Então o coeficiente Spearman $\rho$ para $(X, Y)$ é dado por:

$$
\rho(X, Y)=3 Q(C, \Pi)=12 \iint_{[0,1]^{2}} u v d C(u, v)-3=12 \iint_{[0,1]^{2}} C(u, v) d u d v-3 .
$$


Teorema 1.9. [Embrechts et al. (2002)] Sejam $X$ e $Y$ variáveis aleatórias contínuas com cópula $C$, e seja $\kappa$ a medida Kendall $\tau$ ou Spearman $\rho$. Então

$$
\kappa(X, Y)=1 \Leftrightarrow C=M \text { e } \kappa(X, Y)=-1 \Leftrightarrow C=W
$$

Das definições de Kendall $\tau$ e Spearman $\rho$ segue que ambas são funções crescentes do valor da cópula considerada. Além disso, para variáveis aleatórias contínuas todos os valores possíveis (o intervalo $[-1,1]$ ) das medidas Kendall $\tau$ e Spearman $\rho$ podem ser obtidos através de uma mudança adequada da cópula em questão.

No teorema a seguir as versões amostrais das medidas Kendall $\tau$ e Spearman $\rho$ são formuladas.

Teorema 1.10. [Nelsen (2006)]. Seja $C_{n}\left(\frac{i}{n}, \frac{j}{n}\right)$ a cópula empírica da amostra $\left\{\left(x_{k}, y_{k}\right)\right\}_{k=1}^{n}$ do vetor aleatório contínuo $(X, Y)$, dada por:

$$
C_{n}\left(\frac{i}{n}, \frac{j}{n}\right)=\frac{1}{n}\left\{\text { número de pontos }\left(x_{k}, y_{k}\right) \text { tais que } x_{k} \leq x_{(i)} \text { e } y_{k} \leq y_{(j)}\right\} \text {, }
$$

onde $(i)$ denota a $i$-ésima estatística de ordem e seja $c_{n}\left(\frac{i}{n}, \frac{j}{n}\right)$ a freqüência associada à cópula empírica, dada por:

$$
c_{n}\left(\frac{i}{n}, \frac{j}{n}\right)= \begin{cases}\frac{1}{n}, & \text { se }\left(x_{(i)}, y_{(j)}\right) \text { é um elemento da amostra, } \\ 0, & \text { caso contrário. }\end{cases}
$$

Então as versões amostrais dos coeficientes Kendall $\tau$ e Spearman $\rho, t$ e $r$, respectivamente, são dadas por:

$$
\begin{aligned}
& t=\frac{2 n}{n-1} \sum_{i=2}^{n} \sum_{j=2}^{n} \sum_{p=1}^{i-1} \sum_{q=1}^{j-1}\left[c_{n}\left(\frac{i}{n}, \frac{j}{n}\right) c_{n}\left(\frac{p}{n}, \frac{q}{n}\right)-c_{n}\left(\frac{i}{n}, \frac{q}{n}\right) c_{n}\left(\frac{p}{n}, \frac{j}{n}\right)\right] \mathrm{e} \\
& r=\frac{12}{n^{2}-1} \sum_{i=1}^{n} \sum_{j=1}^{n}\left[C_{n}\left(\frac{i}{n}, \frac{j}{n}\right)-\frac{i}{n} \frac{j}{n}\right] .
\end{aligned}
$$




\section{Capítulo 2.}

\section{Conceitos e Medidas de Dependência Local}

Neste capítulo tratamos o fenômeno da dependência local, sendo discutidas algumas medidas já existentes na literatura, e duas novas medidas, baseadas na distribuição condicional dos dados, são propostas. Algumas propriedades e aplicações dessas medidas são analisadas

\subsection{Algumas Medidas Locais de Dependência}

O fenômeno da dependência local tem recebido crescente atenção de muitos autores nos últimos anos, a exemplo de Jones (1996) e Schmid e Schmidt (2007). Isso advém da percepção que as medidas globais de dependência resumem demasiadamente a informação sobre a dependência implícita nos dados, basicamente por traduzir toda a estrutura subjacente em um único número. Algumas alternativas para contornar essa deficiência têm sido propostas na literatura, visando basicamente analisar a dependência localmente via condicionamento dos dados analisados tanto em uma porção reduzida de seu espaço amostral, quanto em pontos específicos do mesmo. Algumas das medidas locais de dependência mais utilizadas e citadas na literatura estatística são: a função de dependência local estudada em Holland e Wang (1987) e a medida local de dependência linear proposta em Bairamov et al. (2003). Em ambos os casos as medidas tratam de pontos específicos do espaço amostral. A seguir, essas medidas, e algumas de suas propriedades, são apresentadas formalmente.

\subsubsection{Função de Dependência Local}

A primeira medida estudada de dependência local a ser apresentada nesta tese é a função de dependência local para variáveis aleatórias contínuas, definida em Holland e Wang (1987) como

$$
\gamma(x, y)=\frac{\partial^{2} \ln (h(x, y))}{\partial x \partial y}
$$

onde $h(x, y)$ representa a função densidade conjunta contínua das variáveis $X$ e $Y$.

Seguem algumas propriedades da função de dependência local $\gamma(x, y)$ :

(i) $\quad \gamma(x, y)=0$ se e somente se $X$ e $Y$ são independentes;

(ii) $\quad \gamma(x, y)$ não depende das distribuições marginais de $X$ e $Y$, sendo função apenas da distribuição condicional de $X$ dado $Y$ ou de $Y$ dado $X$;

(iii) Sob condições técnicas usuais, há uma única função densidade $h(x, y)$ ligando a função de dependência local $\gamma(x, y)$ às densidades marginais $f(x)$ e $g(y)$ de $X$ e $Y$, respectivamente. 
Segue um exemplo de aplicação da função de dependência local $\gamma(x, y)$ à distribuição Normal bivariada:

Seja o vetor $(X, Y)$ com distribuição Normal bivariada $\mathcal{N}\left(\mu_{X}, \mu_{Y}, \sigma_{X}, \sigma_{Y}, \rho_{X, Y}\right)$. Então sua função densidade conjunta e a correspondente função de dependência local têm as seguintes expressões:

$$
\begin{aligned}
& h(x, y)=\frac{1}{2 \pi \sigma_{X} \sigma_{Y} \sqrt{1-\rho_{X, Y}^{2}}} \exp \left\{-\frac{1}{2\left(1-\rho_{X, Y}^{2}\right)}\left[\left(\frac{x-\mu_{X}}{\sigma_{X}}\right)^{2}-2 \rho_{X, Y}\left(\frac{x-\mu_{X}}{\sigma_{X}}\right)\left(\frac{y-\mu_{Y}}{\sigma_{Y}}\right)+\left(\frac{y-\mu_{Y}}{\sigma_{Y}}\right)^{2}\right]\right\} ; \\
& \frac{\partial \ln (h(x, y))}{\partial y}=-\frac{1}{2\left(1-\rho_{X, Y}^{2}\right)}\left[-2 \rho_{X, Y}\left(\frac{x-\mu_{X}}{\sigma_{X} \sigma_{Y}}\right)+2\left(\frac{y-\mu_{Y}}{\sigma_{Y}}\right)\right] ; \\
& \gamma(x, y)=\frac{\partial^{2} \ln (h(x, y))}{\partial x \partial y}=\frac{\rho_{X, Y}}{\left(1-\rho_{X, Y}^{2}\right) \sigma_{X} \sigma_{Y}} .
\end{aligned}
$$

Podemos observar que neste caso a função de dependência local é constante para todos os pontos $(x, y)$.

Observação. A função de dependência local é a transposição para o caso contínuo do conceito de "Cross-product ratio", que são medidas de dependência usadas em tabelas de contingência. A definição do "Cross-product ratio" é a seguinte:

Seja uma tabela de contingência bidimensional $\left[P_{i, j}\right]$ que descreve a função de probabilidade conjunta de duas variáveis categorizadas. A informação sobre a associação entre essas variáveis está plenamente contida nos "Cross-product ratios" locais, definidos por

$$
\alpha_{i, j}=\frac{P_{i, j} P_{i+1, j+1}}{P_{i, j+1} P_{i+1, j}}
$$

para todos $i$ e $j$ onde o índice esteja bem definido.

A informação contida nesses índices conjuntamente com as distribuições marginais especifica unicamente a tabela de contingência (ver Goodman (1969) para maiores detalhes).

\subsubsection{Medida Local de Dependência Linear de Bairamov e Kotz}

Uma alternativa para mensurar localmente a associação entre variáveis aleatórias é a medida local de dependência linear de Bairamov e Kotz, que é definida da seguinte maneira:

$$
B K(x, y)=\frac{E([X-E(X \mid Y=y)][Y-E(Y \mid X=x)])}{\sqrt{E\left([X-E(X \mid Y=y)]^{2}\right) E\left([Y-E(Y \mid X=x)]^{2}\right)}}
$$


Seguem algumas propriedades básicas da medida local de dependência linear $B K(x, y)$ :

(i) Se $X$ e $Y$ são independentes, então $B K(x, y)=0$ para todo ponto $(x, y)$ pertencente ao suporte da função distribuição conjunta de $(X, Y)$;

(ii) $|B K(x, y)| \leq 1$ para todo ponto $(x, y)$ pertencente ao suporte da função distribuição conjunta de $(X, Y)$;

(iii) Se $B K(x, y)= \pm 1$ para algum $(x, y)$ pertencente ao suporte da função distribuição conjunta de $(X, Y)$, então $\rho_{X, Y} \neq 0$;

(iv) Se $\rho_{X, Y}= \pm 1$, então $B K(x, y)= \pm 1$ quase certamente.

Para outras propriedades de $B K(x, y)$ e resultados adicionais ver Bairamov et al. (2003).

Segue um exemplo de aplicação para fins de comparação com a função de dependência local para a distribuição Normal bivariada. Seja o vetor $(X, Y)$ com distribuição Normal bivariada $\mathcal{N}\left(\mu_{X}, \mu_{Y}, \sigma_{X}, \sigma_{Y}, \rho_{X, Y}\right)$. Então $B K(x, y)$ tem a seguinte expressão:

$$
B K(x, y)=\frac{\sigma_{X} \sigma_{Y} \rho_{X, Y}+\rho_{X, Y}^{2}\left(x-\mu_{X}\right)\left(y-\mu_{Y}\right)}{\sqrt{\left[\sigma_{X}^{2}+\rho_{X, Y}^{2}\left(x-\mu_{X}\right)^{2}\right]} \sqrt{\left[\sigma_{Y}^{2}+\rho_{X, Y}^{2}\left(y-\mu_{Y}\right)^{2}\right]}}
$$

Podemos notar que, ao contrário da função de dependência local, no caso normal bivariado a medida local de dependência linear é dependente do ponto $(x, y)$.

Algumas observações a respeito das medidas tratadas anteriormente são importantes:

1) Ambas são medidas pontuais de dependência;

2) As duas medidas estão fundamentadas em quantidades de difícil obtenção na vida prática: a função de densidade conjunta no caso da função de dependência local e as esperanças condicionais no caso da medida de Bairamov e Kotz;

3) Ambas estão sujeitas a restrições que muitos dados reais apresentam: a necessidade da função densidade conjunta contínua e derivável, não singular no caso da função de dependência local e a necessidade de a distribuição conjunta ter os segundos momentos finitos no caso da medida de Bairamov e Kotz;

4) A medida de Bairamov e Kotz apresenta o mesmo problema do coeficiente de correlação linear de Pearson que é ser uma medida dependente das distribuições marginais envolvidas.

Dessa forma, o intuito desta investigação é propor medidas de dependência local que contornem essas dificuldades e tenham boas propriedades estatísticas. 


\subsection{Distribuição Condicional dos Dados e Dependência Local}

Seja $C(u, v)$ a cópula descrevendo a relação de dependência das variáveis $U$ e $V$ uniformemente distribuídas em $(0,1)$. No sentido de estudarmos medidas de dependência local, é importante analisarmos as distribuições conjuntas condicionais:

$$
L_{C}(u, v)=P\left(U \leq u, V \leq v \mid(U, V) \in\left[u_{0}, u_{1}\right] \times\left[v_{0}, v_{1}\right]\right)
$$

para $0 \leq u_{0}<u_{1} \leq 1$ e $0 \leq v_{0}<v_{1} \leq 1$

Temos, portanto, que para $(u, v) \in\left[u_{0}, u_{1}\right] \times\left[v_{0}, v_{1}\right]$ :

$$
\begin{aligned}
L_{C}(u, v) & =\frac{P\left(u_{0} \leq U \leq u, v_{0} \leq V \leq v\right)}{P\left(u_{0} \leq U \leq u_{1}, v_{0} \leq V \leq v_{1}\right)} \\
& =\frac{C(u, v)-C\left(u_{0}, v\right)-C\left(u, v_{0}\right)+C\left(u_{0}, v_{0}\right)}{C\left(u_{1}, v_{1}\right)-C\left(u_{0}, v_{1}\right)-C\left(u_{1}, v_{0}\right)+C\left(u_{0}, v_{0}\right)}
\end{aligned}
$$

A expressão acima faz sentido quando o denominador é maior que zero, ou seja, $C\left(u_{1}, v_{1}\right)-C\left(u_{0}, v_{1}\right)-C\left(u_{1}, v_{0}\right)+C\left(u_{0}, v_{0}\right)>0$, sendo definida como zero nos casos onde a desigualdade não se verifica.

As distribuições marginais de $L_{C}(u, v)$ são:

$$
\begin{aligned}
& L_{U}(u)=\frac{C\left(u, v_{1}\right)-C\left(u_{0}, v_{1}\right)-C\left(u, v_{0}\right)+C\left(u_{0}, v_{0}\right)}{C\left(u_{1}, v_{1}\right)-C\left(u_{0}, v_{1}\right)-C\left(u_{1}, v_{0}\right)+C\left(u_{0}, v_{0}\right)} \\
& L_{V}(v)=\frac{C\left(u_{1}, v\right)-C\left(u_{0}, v\right)-C\left(u_{1}, v_{0}\right)+C\left(u_{0}, v_{0}\right)}{C\left(u_{1}, v_{1}\right)-C\left(u_{0}, v_{1}\right)-C\left(u_{1}, v_{0}\right)+C\left(u_{0}, v_{0}\right)} .
\end{aligned}
$$

Como se pode notar pelas expressões acima, $L_{U}(u)$ e $L_{V}(v)$ não caracterizam, de maneira geral, distribuições uniformes e, portanto, $L_{C}(u, v)$ não é uma cópula. A obtenção da cópula subjacente pode ser feita através da relação

$$
C_{L}(u, v)=L_{C}\left(L_{U}^{-1}(u), L_{V}^{-1}(v)\right)
$$

No entanto, por facilidade de cálculo, no que segue serão usadas as distribuições condicionais $L_{C}(u, v)$ ao invés de suas cópulas subjacentes.

\subsection{As Versões Locais dos Coeficientes Kendall $\tau$ e Spearman $\rho$}

Nesta seção definiremos as versões locais dos coeficientes $\rho$ e $\tau$ baseadas nas distribuições condicionais definidas acima, utilizando-se para isso as versões amostrais de tais coeficientes. 
Definição. Sejam $\left(U_{1}, V_{1}\right),\left(U_{2}, V_{2}\right)$ e $\left(U_{3}, V_{3}\right)$ cópias independentes do vetor $(U, V)$ de variáveis $U(0,1)$ com cópula $C(u, v)$ e $\left[u_{0}, u_{1}\right] \times\left[v_{0}, v_{1}\right]$ um subconjunto do quadrado unitário $[0,1]^{2}$ tal que $u_{0}<u_{1}$ e $v_{0}<v_{1}$. Então as versões locais de $\rho$ e $\tau$ no retângulo $\left[u_{0}, u_{1}\right] \mathrm{x}\left[v_{0}, v_{1}\right]$ são definidas como:

$$
\begin{aligned}
& \begin{aligned}
\rho_{L_{C}}\left(\left[u_{0}, u_{1}\right] \mathrm{x}[\right. & \left.\left.v_{0}, v_{1}\right]\right) \\
\quad & =3 P\left(\left(U_{1}-U_{2}\right)\left(V_{1}-V_{3}\right)>0 \mid\left(U_{i}, V_{i}\right) \in\left[u_{0}, u_{1}\right] \mathrm{x}\left[v_{0}, v_{1}\right], i=1,2,3 .\right) \\
& -3 P\left(\left(U_{1}-U_{2}\right)\left(V_{1}-V_{3}\right)<0 \mid\left(U_{i}, V_{i}\right) \in\left[u_{0}, u_{1}\right] \mathrm{x}\left[v_{0}, v_{1}\right], i=1,2,3 .\right)
\end{aligned} \\
& \mathrm{e} \\
& \begin{aligned}
\tau_{L_{C}}\left(\left[u_{0}, u_{1}\right] \mathrm{x}\left[v_{0}, v_{1}\right]\right)=2 P\left(\left(U_{1}-U_{2}\right)\left(V_{1}-V_{2}\right)>0 \mid\left(U_{i}, V_{i}\right) \in\left[u_{0}, u_{1}\right] \mathrm{x}\left[v_{0}, v_{1}\right], i=1,2 .\right)-1
\end{aligned}
\end{aligned}
$$

Esses coeficientes objetivam evidenciar a dependência entre variáveis aleatórias em uma porção reduzida de seu espaço amostral. As proposições abaixo enunciam algumas propriedades dessas medidas:

Proposição 2.1. São válidas as seguintes relações:

$$
\rho_{L_{C}}\left(\left[u_{0}, u_{1}\right] \mathrm{x}\left[v_{0}, v_{1}\right]\right)=12 \int_{v_{0}}^{v_{1}} \int_{u_{0}}^{u_{1}} L_{C}(u, v) d L_{U}(u) d L_{V}(v)-3
$$

e

$$
\tau_{L_{C}}\left(\left[u_{0}, u_{1}\right] \mathrm{x}\left[v_{0}, v_{1}\right]\right)=4 \int_{v_{0}}^{v_{1}} \int_{u_{0}}^{u_{1}} L_{C}(u, v) d L_{C}(u, v)-1
$$

Prova: Aplicando a distribuição condicional de $(U, V)$ dado que $(U, V) \in\left[u_{0}, u_{1}\right] \mathrm{x}\left[v_{0}, v_{1}\right]$ desenvolvida na seção 2.2 às versões populacionais dos coeficientes Kendall $\tau$ e Spearman $\rho$ definidas por

$$
\rho=12 \int_{-\infty}^{\infty} \int_{-\infty}^{\infty} H(x, y) d F(x) d G(y)-3
$$

e

$$
\tau=4 \int_{-\infty}^{\infty} \int_{-\infty}^{\infty} H(x, y) d H(x, y)-1
$$

onde $H(x, y)$ representa a função distribuição do vetor aleatório contínuo $(X, Y)$ com marginais $F$ e $G$, de $X$ e $Y$ respectivamente, e que tem cópula $C(u, v)$, temos que o resultado segue. 
Proposição 2.2. $\tau=E\left(\tau_{L_{C}}\left(\left[u_{0}, u_{1}\right] \times\left[v_{0}, v_{1}\right]\right)\right)$ e $\rho=E\left(\rho_{L_{C}}\left(\left[u_{0}, u_{1}\right] \times\left[v_{0}, v_{1}\right]\right)\right)$.

Prova: Temos que

$$
\begin{aligned}
& \tau=2 P\left(\left(U_{1}-U_{2}\right)\left(V_{1}-V_{2}\right)>0\right)-1 \text {; } \\
& =2 E\left(E\left(\mathbb{I}\left(\left(U_{1}-U_{2}\right)\left(V_{1}-V_{2}\right)>0 \mid\left(U_{i}, V_{i}\right) \in\left[u_{0}, u_{1}\right] \mathrm{x}\left[v_{0}, v_{1}\right], i=1,2 .\right)\right)\right) \\
& =2 E\left(\frac{\tau_{L_{C}}\left(\left[u_{0}, u_{1}\right] \times\left[v_{0}, v_{1}\right]\right)+1}{2}\right)-1 \\
& =E\left(\tau_{L_{C}}\left(\left[u_{0}, u_{1}\right] \mathrm{x}\left[v_{0}, v_{1}\right]\right)\right) \mathrm{e} \\
& \rho=3\left[P\left(\left(U_{1}-U_{2}\right)\left(V_{1}-V_{3}\right)>0\right)-P\left(\left(U_{1}-U_{2}\right)\left(V_{1}-V_{3}\right)<0\right)\right] \\
& =3\left[\begin{array}{c}
E\left(E\left(\mathbb{1}\left(\left(U_{1}-U_{2}\right)\left(V_{1}-V_{3}\right)>0 \mid\left(U_{i}, V_{i}\right) \in\left[u_{0}, u_{1}\right] \mathrm{x}\left[v_{0}, v_{1}\right], i=1,2,3 .\right)\right)\right) \\
-E\left(E\left(\mathbb{1}\left(\left(U_{1}-U_{2}\right)\left(V_{1}-V_{3}\right)<0 \mid\left(U_{i}, V_{i}\right) \in\left[u_{0}, u_{1}\right] \mathrm{x}\left[v_{0}, v_{1}\right], i=1,2,3 .\right)\right)\right)
\end{array}\right] ; \\
& =3 E\left(\frac{\rho_{L_{C}}\left(\left[u_{0}, u_{1}\right] \times\left[v_{0}, v_{1}\right]\right)}{3}\right) \\
& =E\left(\rho_{L_{C}}\left(\left[u_{0}, u_{1}\right] \mathrm{x}\left[v_{0}, v_{1}\right]\right)\right) .
\end{aligned}
$$

A última proposição mostra que as versões globais das medidas Kendall $\tau$ e Spearman $\rho$ são uma média ponderada das versões locais, onde os pesos são as massas de probabilidades dos subconjuntos $\left[u_{0}, u_{1}\right] \times\left[v_{0}, v_{1}\right]$.

Proposição 2.3. As seguintes desigualdades são válidas:

$$
\left|\rho_{L_{C}}\left(\left[u_{0}, u_{1}\right] \mathrm{x}\left[v_{0}, v_{1}\right]\right)\right|,\left|\tau_{L_{C}}\left(\left[u_{0}, u_{1}\right] \mathrm{x}\left[v_{0}, v_{1}\right]\right)\right| \leq 1
$$

Prova: Como $L_{C}(u, v)$ é uma função distribuição, o resultado segue da proposição 2.1 e das propriedades de $\rho$ e $\tau$.

Proposição 2.4. Se $C(u, v) \in\{W(u, v), \Pi(u, v), M(u, v)\}$, então $\rho=\rho_{L_{C}}\left(\left[u_{0}, u_{1}\right] \mathrm{x}\left[v_{0}, v_{1}\right]\right) \mathrm{e}$ $\tau=\tau_{L_{C}}\left(\left[u_{0}, u_{1}\right] \mathrm{x}\left[v_{0}, v_{1}\right]\right)$. Adicionalmente, para esses mesmos casos a cópula condicional é idêntica à incondicional. 
Prova: Serão demonstrados os casos para $M(u, v)$ e $\Pi(u, v)$. O caso para $W(u, v)$ é análogo ao de $M(u, v)$. Seja $C(u, v)=\Pi(u, v)=u v$. Temos que

$$
L_{\Pi}(u, v)=\frac{u v-u v_{0}-u_{0} v+u_{0} v_{0}}{u_{1} v_{1}-u_{1} v_{0}-u_{0} v_{1}+u_{0} v_{0}}
$$

As distribuições marginais são as seguintes:

$$
L_{U}(u)=\frac{u-u_{0}}{u_{1}-u_{0}} \text { e } L_{V}(v)=\frac{v-v_{0}}{v_{1}-v_{0}}
$$

e a cópula subjacente, $C_{L}(u, v)$, é obtida através da relação $C_{L}(u, v)=L_{C}\left(L_{U}^{-1}(u), L_{V}^{-1}(v)\right)$.

Temos então que $C_{L}(u, v)=C_{\Pi}(u, v)=u v$.

Portanto, para o caso de independência, a cópula condicional também é a cópula de independência, como seria natural esperar. Segue, portanto, que:

$$
\rho=\rho_{L_{\Pi}}\left(\left[u_{0}, u_{1}\right] \mathrm{x}\left[v_{0}, v_{1}\right]\right)=0 \text { e } \tau=\tau_{L_{\Pi}}\left(\left[u_{0}, u_{1}\right] \mathrm{x}\left[v_{0}, v_{1}\right]\right)=0
$$

Seja $C(u, v)=M(u, v)=\operatorname{Min}(u, v)$. Temos que

$$
\tau_{L_{M}}\left(\left[u_{0}, u_{1}\right] \mathrm{x}\left[v_{0}, v_{1}\right]\right)=4 \int_{v_{0}}^{v_{1}} \int_{u_{0}}^{u_{1}} M(u, v) d M(u, v)-1
$$

Tomando $V_{M}(0,1)=M\left(u_{1}, v_{1}\right)-M\left(u_{0}, v_{1}\right)-M\left(u_{1}, v_{0}\right)+M\left(u_{0}, v_{0}\right)$, temos então:

$$
\begin{aligned}
\tau_{L_{M}}\left(\left[u_{0}, u_{1}\right] \mathrm{x}\left[v_{0}, v_{1}\right]\right) & \\
= & \frac{4}{V_{M}^{2}(0,1)}\left\{\frac{\operatorname{Min}^{2}\left(u_{1}, v_{1}\right)}{2}-\frac{\operatorname{Max}^{2}\left(u_{0}, v_{0}\right)}{2}\right. \\
& \left.-\left(u_{0}+v_{0}\right)\left[\operatorname{Min}\left(u_{1}, v_{1}\right)-\operatorname{Max}\left(u_{0}, v_{0}\right)\right]+\operatorname{Min}\left(u_{0}, v_{0}\right) V_{M}(0,1)\right\}-1
\end{aligned}
$$

Há seis casos a serem analisados:

1. $u_{0}<u_{1}<v_{0}<v_{1}$. Neste caso $V_{M}(0,1)=0$ e, portanto, não cabe nenhuma análise.

2. $u_{0}<v_{0}<u_{1}<v_{1}$. Neste caso $\tau_{L_{M}}\left(\left[u_{0}, u_{1}\right] \mathrm{x}\left[v_{0}, v_{1}\right]\right)=1$.

3. $v_{0}<u_{0}<u_{1}<v_{1}$. Neste caso $\tau_{L_{M}}\left(\left[u_{0}, u_{1}\right] \mathrm{x}\left[v_{0}, v_{1}\right]\right)=1$.

4. $v_{0}<u_{0}<v_{1}<u_{1}$. Neste caso $\tau_{L_{M}}\left(\left[u_{0}, u_{1}\right] \mathrm{x}\left[v_{0}, v_{1}\right]\right)=1$. 
5. $v_{0}<v_{1}<u_{0}<u_{1}$. Neste caso $V_{M}(0,1)=0$ e, portanto, não cabe nenhuma análise.

6. $u_{0}<v_{0}<v_{1}<u_{1}$. Neste caso $\tau_{L_{M}}\left(\left[u_{0}, u_{1}\right] \mathrm{x}\left[v_{0}, v_{1}\right]\right)=1$.

Portanto, para todos os pontos $(u, v)$ onde $L_{M}(u, v)>0, \tau_{L_{M}}\left(\left[u_{0}, u_{1}\right] \mathrm{x}\left[v_{0}, v_{1}\right]\right)=\tau=1$. Como $\tau(U, V)=1 \Leftrightarrow C=M$, temos que $C_{M}(u, v)=M(u, v)$, ou seja, a cópula subjacente à distribuição condicional também é a cópula comonotônica. Disso segue também que

$$
\rho_{L_{M}}\left(\left[u_{0}, u_{1}\right] \mathrm{x}\left[v_{0}, v_{1}\right]\right)=\rho=1
$$

Proposição 2.5. Se $V=f(U)$, sendo $f$ uma função contínua e diferenciável, então

$$
\rho_{L_{C}}\left(\left[u_{0}, u_{1}\right] \mathrm{x}\left[v_{0}, v_{1}\right]\right), \tau_{L_{C}}\left(\left[u_{0}, u_{1}\right] \mathrm{x}\left[v_{0}, v_{1}\right]\right) \in\{-1,0,1\}
$$

Prova: Da proposição 2.4 e das propriedades de cópulas temos três casos distintos:

1. Regiões onde $f$ é crescente.

$C_{L}(u, v)=M(u, v)$, e portanto $\rho_{L_{C}}\left(\left[u_{0}, u_{1}\right] \mathrm{x}\left[v_{0}, v_{1}\right]\right)=\tau_{L_{C}}\left(\left[u_{0}, u_{1}\right] \mathrm{x}\left[v_{0}, v_{1}\right]\right)=1$.

2. Regiões onde $f$ é constante.

$C_{L}(u, v)=\Pi(u, v)$ e, portanto, $\rho_{L_{C}}\left(\left[u_{0}, u_{1}\right] \mathrm{x}\left[v_{0}, v_{1}\right]\right)=\tau_{L_{C}}\left(\left[u_{0}, u_{1}\right] \mathrm{x}\left[v_{0}, v_{1}\right]\right)=0$.

3. Regiões onde $f$ é decrescente.

$C_{L}(u, v)=W(u, v)$ e, portanto, $\rho_{L_{C}}\left(\left[u_{0}, u_{1}\right] x\left[v_{0}, v_{1}\right]\right)=\tau_{L_{C}}\left(\left[u_{0}, u_{1}\right] x\left[v_{0}, v_{1}\right]\right)=-1$

Proposição 2.6. Sejam $\left\{\left(x_{k}, y_{k}\right)\right\}_{k=1}^{n}$ uma amostra do vetor aleatório contínuo $(X, Y), \breve{F}(x)$ e $\check{G}(y)$ as distribuições marginais empíricas de $X$ e $Y$, respectivamente, $S\left(\left[u_{0}, u_{1}\right] \mathrm{x}\left[v_{0}, v_{1}\right]\right)=\left\{\left(x_{k}, y_{k}\right)\right.$ tais que $u_{0} \leq \breve{F}\left(x_{k}\right) \leq u_{1}$ e $\left.v_{0} \leq \check{G}\left(y_{k}\right) \leq v_{1}, k=1, \ldots, n\right\}$ o subconjunto de pares da amostra que estão dentro da área condicionada $\left[u_{0}, u_{1}\right] \mathrm{x}\left[v_{0}, v_{1}\right] \mathrm{e}$ $s=\left|S\left(\left[u_{0}, u_{1}\right] \mathrm{x}\left[v_{0}, v_{1}\right]\right)\right|$ a cardinalidade do conjunto $S\left(\left[u_{0}, u_{1}\right] \mathrm{x}\left[v_{0}, v_{1}\right]\right)$. Expressando $S$ como $S\left(\left[u_{0}, u_{1}\right] \mathrm{x}\left[v_{0}, v_{1}\right]\right)=\left\{\left(x_{k}^{\prime}, y_{k}^{\prime}\right)\right\}_{k=1}^{s}$, segue que a cópula empírica $\breve{C}_{s,\left[u_{0}, u_{1}\right] \mathrm{x}\left[v_{0}, v_{1}\right]}\left(\frac{i}{s}, \frac{j}{s}\right)$ para o conjunto $\left\{\left(x_{k}^{\prime}, y_{k}^{\prime}\right)\right\}_{k=1}^{s}$ é dada por:

$$
\breve{C}_{s,\left[u_{0}, u_{1}\right] \mathrm{x}\left[v_{0}, v_{1}\right]}\left(\frac{i}{s}, \frac{j}{s}\right)=\frac{1}{s}\left\{\text { número de pontos }\left(x_{k}^{\prime}, y_{k}^{\prime}\right) \text { tais que } x_{k}^{\prime} \leq x_{(i)}^{\prime} \text { e } y_{k}^{\prime} \leq y_{(j)}^{\prime}\right\}
$$


onde $(i)$ denota a $i$-ésima estatística de ordem e que $\breve{c}_{s,\left[u_{0}, u_{1}\right] \times\left[v_{0}, v_{1}\right]}$ é a freqüência da cópula empírica $\breve{C}_{s,\left[u_{0}, u_{1}\right] \mathrm{x}\left[v_{0}, v_{1}\right]}\left(\frac{i}{s}, \frac{j}{s}\right)$, sendo dada por:

$$
\breve{c}_{s,\left[u_{0}, u_{1}\right] \times\left[v_{0}, v_{1}\right]}= \begin{cases}\frac{1}{s}, & \text { se }\left(x_{(i)}^{\prime}, y_{(j)}^{\prime}\right) \text { é um elemento do conjunto }\left\{\left(x_{k}^{\prime}, y_{k}^{\prime}\right)\right\}_{k=1}^{s}, \\ 0, & \text { caso contrário. }\end{cases}
$$

Então as versões amostrais dos coeficientes locais Kendall $\tau$ e Spearman $\rho$, $t_{L_{C}}\left(\left[u_{0}, u_{1}\right] \mathrm{x}\left[v_{0}, v_{1}\right]\right)$ e $r_{L_{C}}\left(\left[u_{0}, u_{1}\right] \mathrm{x}\left[v_{0}, v_{1}\right]\right)$, respectivamente, são dados por:

$$
\begin{gathered}
t_{L_{C}}\left(\left[u_{0}, u_{1}\right] \times\left[v_{0}, v_{1}\right]\right) \\
=\frac{2 s}{s-1} \sum_{i=2}^{s} \sum_{j=2}^{s} \sum_{p=1}^{i-1} \sum_{q=1}^{j-1}\left[\breve{c}_{s,\left[u_{0}, u_{1}\right] \times\left[v_{0}, v_{1}\right]}\left(\frac{i}{s}, \frac{j}{s}\right) \breve{c}_{s,\left[u_{0}, u_{1}\right] \times\left[v_{0}, v_{1}\right]}\left(\frac{p}{s}, \frac{q}{s}\right)\right. \\
\left.-\breve{c}_{s,\left[u_{0}, u_{1}\right] \times\left[v_{0}, v_{1}\right]}\left(\frac{i}{s}, \frac{q}{s}\right) \breve{c}_{s,\left[u_{0}, u_{1}\right] \times\left[v_{0}, v_{1}\right]}\left(\frac{p}{s}, \frac{j}{s}\right)\right] \mathrm{e} \\
r_{L_{C}}\left(\left[u_{0}, u_{1}\right] \times\left[v_{0}, v_{1}\right]\right)=\frac{12}{s^{2}-1} \sum_{i=1}^{n} \sum_{j=1}^{n}\left[\breve{C}_{s,\left[u_{0}, u_{1}\right] \times\left[v_{0}, v_{1}\right]}\left(\frac{i}{s}, \frac{j}{s}\right)-\frac{i}{s} \frac{j}{s}\right] .
\end{gathered}
$$

Prova: Aplicando as definições dos coeficientes locais Kendall $\tau$ e Spearman $\rho$, a proposição 2.1 e o teorema 1.10, temos que o resultado segue.

\subsection{Aplicações a Algumas Famílias de Cópulas}

Daremos nesta subseção alguns exemplos de como as versões locais dos coeficientes de Kendall e Spearman se comportam para algumas famílias de cópulas.

\subsubsection{Família Farlie-Gumbel-Morgenstern}

Seja uma cópula $C(u, v)$ pertencente à família Farlie-Gumbel-Morgenstern, isto é, de forma: $C_{\theta}(u, v)=u v+\theta u v(1-u)(1-v), \operatorname{com} \theta \in[-1,1]$.

Calculando a distribuição condicional $L_{C}(u, v)$ e resolvendo as integrais com o software Mathematica 5.1 [Wolfram Research], temos que os coeficientes locais de Kendall $\left(\tau_{L_{C}}\right)$ e Spearman $\left(\rho_{L_{C}}\right)$ são dados pelas seguintes equações:

$$
\begin{aligned}
\tau_{L_{C}}\left(\left[u_{0}, u_{1}\right] \mathrm{x}\left[v_{0}, v_{1}\right]\right) & =\frac{2\left(u_{1}-u_{0}\right)\left(v_{1}-v_{0}\right) \theta}{9\left[1+\left(1-u_{1}-u_{0}\right)\left(1-v_{1}-v_{0}\right) \theta\right]^{2}}, \\
\rho_{L_{C}}\left(\left[u_{0}, u_{1}\right] \times\left[v_{0}, v_{1}\right]\right) & =\frac{\left(u_{1}-u_{0}\right)\left(v_{1}-v_{0}\right) \theta}{3\left[1+\left(1-u_{1}-u_{0}\right)\left(1-v_{1}-v_{0}\right) \theta\right]^{2}} .
\end{aligned}
$$


Temos, portanto, que $\tau_{L_{C}}\left(\left[u_{0}, u_{1}\right] \mathrm{x}\left[v_{0}, v_{1}\right]\right)=\frac{2}{3} \rho_{L_{C}}\left(\left[u_{0}, u_{1}\right] \mathrm{x}\left[v_{0}, v_{1}\right]\right)$, quaisquer que sejam o parâmetro $\theta$ e o intervalo $\left[u_{0}, u_{1}\right] \times\left[v_{0}, v_{1}\right]$ tomados. O interessante é que essa relação é bem conhecida no caso global, mas que permanece válida quando olhamos a dependência localmente. Essa característica é particular dessa cópula, não sendo uma propriedade geral. Dividindo-se o quadrado unitário em quatro partes iguais temos, graficamente, a seguinte variação das medidas locais, para cada sub-quadrado, em função do parâmetro $\theta$.

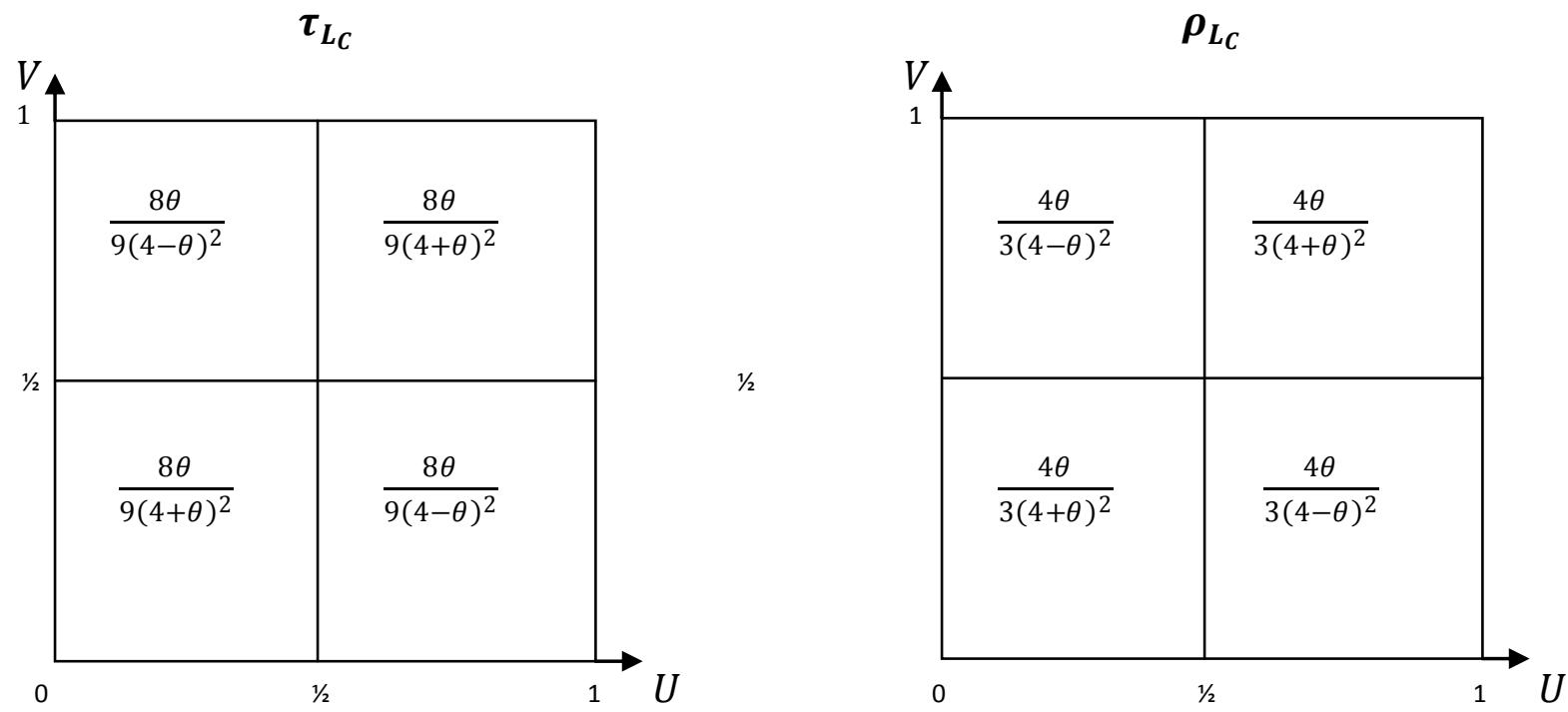

Figura 2.1. Comparação de $\tau_{L_{C}}$ e $\rho_{L_{C}}$ para a família Farlie-Gumbel-Morgenstern de cópulas.

Em Fredricks e Nelsen (2007) os autores estudam as relações entre os coeficientes Kendall $\tau$ e Spearman $\rho$ para uma variedade de cópulas, bem como desigualdades gerais entre os mesmos. Neste artigo é demonstrado que o limite da razão $\frac{\rho}{\tau}$ se aproxima de $\frac{3}{2}$ quando a função distribuição das variáveis aleatórias se aproxima da independência.

\subsubsection{Família de Cópulas Singulares}

A família tratada nesta seção é aquela onde as cópulas integrantes têm seu suporte como uma coleção de segmentos de coeficiente angular positivo ou negativo. Da proposição 2.4, temos que quando o suporte é um segmento de coeficiente angular positivo a cópula condicional implícita é a de comonotonicidade, ou cópula $M(u, v)$. No caso onde o coeficiente angular é negativo a cópula condicional implícita é a de contramonotonicidade, ou cópula $W(u, v)$.

Um caso prático seria a cópula abaixo, muito utilizada para ilustrar a situação de dependência completa entre as variáveis e com medidas globais de dependência (Kendall $\tau$, Spearman $\rho$ e Correlação linear de Pearson) iguais a 0.

Seja:

$$
C(u, v)=\left\{\begin{array}{l}
\operatorname{Max}\left(u+\frac{1}{2}(v-1), 0\right), u \in\left[0, \frac{1}{2}\right) \\
\operatorname{Min}\left(u+\frac{1}{2}(v-1), v\right), u \in\left[\frac{1}{2}, 1\right] .
\end{array}\right.
$$


Essa cópula representa a relação $V=|2 U-1|$. Portanto para $u \in\left[0, \frac{1}{2}\right]$ o suporte é um segmento de coeficiente angular negativo, ligando os pontos $(0,1)$ e $\left(\frac{1}{2}, 0\right)$.

Nos intervalos onde $u_{0}, u_{1} \in\left[0, \frac{1}{2}\right]$ e $C\left(u_{1}, v_{1}\right)-C\left(u_{0}, v_{1}\right)-C\left(u_{1}, v_{0}\right)+C\left(u_{0}, v_{0}\right)>0$, a cópula condicional subjacente é $W(u, v)$ e, portanto,

$$
\rho_{L_{C}}\left(\left[u_{0}, u_{1}\right] \mathrm{x}\left[v_{0}, v_{1}\right]\right)=\tau_{L_{C}}\left(\left[u_{0}, u_{1}\right] \mathrm{x}\left[v_{0}, v_{1}\right]\right)=-1
$$

Nos intervalos onde $u_{0}, u \in\left(\frac{1}{2}, 1\right]$ e $C\left(u_{1}, v_{1}\right)-C\left(u_{0}, v_{1}\right)-C\left(u_{1}, v_{0}\right)+C\left(u_{0}, v_{0}\right)>0$, a cópula condicional subjacente é $M(u, v)$ e, portanto,

$$
\rho_{L_{C}}\left(\left[u_{0}, u_{1}\right] \mathrm{x}\left[v_{0}, v_{1}\right]\right)=\tau_{L_{C}}\left(\left[u_{0}, u_{1}\right] \mathrm{x}\left[v_{0}, v_{1}\right]\right)=1
$$

Aqui podemos verificar a proposição 2.2 , já que

$$
\rho_{L_{C}}\left(\left[u_{0}, u_{1}\right] \mathrm{x}\left[v_{0}, v_{1}\right]\right)=\tau_{L_{C}}\left(\left[u_{0}, u_{1}\right] \mathrm{x}\left[v_{0}, v_{1}\right]\right)=1
$$

e $C\left(u_{1}, v_{1}\right)-C\left(u_{0}, v_{1}\right)-C\left(u_{1}, v_{0}\right)+C\left(u_{0}, v_{0}\right)=\frac{1}{2}$. O mesmo ocorre com

$$
\rho_{L_{C}}\left(\left[u_{0}, u_{1}\right] \mathrm{x}\left[v_{0}, v_{1}\right]\right)=\tau_{L_{C}}\left(\left[u_{0}, u_{1}\right] \mathrm{x}\left[v_{0}, v_{1}\right]\right)=-1
$$

Portanto, os valores globais de $\tau$ e $\rho$ são iguais a 0 , o que pode ser facilmente verificado.

\subsubsection{Família Fréchet-Mardia}

Seguindo Nelsen (2006) e Ferreira (2003), temos que para cópulas $C(u, v)$ definidas por:

$$
C(u, v)=\alpha M(u, v)+\beta \Pi(u, v)+\gamma W(u, v)
$$

para $0 \leq \alpha, \beta, \gamma \leq 1, \alpha+\beta+\gamma=1$, são válidos os seguintes resultados:

$$
\tau_{C}=Q(C, C)=\frac{(\alpha-\gamma)(\alpha+\gamma+2)}{3} \text { e } \rho_{C}=3 Q(C, \Pi)=(\alpha-\gamma)
$$

Pela proposição 2.4 temos que para as cópulas $M$, П e $W$ a cópula condicional é idêntica à não-condicional, fazendo com que esses resultados sejam válidos também se forem consideradas as cópulas condicionais, obtendo-se igualmente que qualquer que seja o intervalo $\left[u_{0}, u_{1}\right] \times\left[v_{0}, v_{1}\right]$ tomado vale que

$$
\tau_{L_{C}}\left(\left[u_{0}, u_{1}\right] \mathrm{x}\left[v_{0}, v_{1}\right]\right)=\frac{(\alpha-\gamma)(\alpha+\gamma+2)}{3} \text { e } \rho_{L_{C}}\left(\left[u_{0}, u_{1}\right] \mathrm{x}\left[v_{0}, v_{1}\right]\right)=(\alpha-\gamma)
$$


Dessa forma, os coeficientes locais e globais são idênticos, e, portanto, para as cópulas da família Fréchet-Mardia, os coeficientes locais Kendall $\tau$ e Spearman $\rho$ são funções basicamente dos pesos que as cópulas $M(u, v)$ e $W(u, v)$ têm na cópula, de acordo com as fórmulas desenvolvidas acima.

\subsection{Comentários}

Algumas observações a respeito das medidas propostas são importantes:

1) Ao contrário das medidas apresentadas anteriormente, as medidas propostas não são medidas pontuais, sendo de mais fácil interpretação e mais intuitivas, por serem versões locais de medidas já consagradas.

2) O cálculo das versões empíricas dessas medidas é idêntico ao caso global, bastando se restringir a região analisada, e não apresenta o inconveniente de se basear em quantidades de difícil obtenção na prática: a função de densidade conjunta no caso da função de dependência local e as esperanças condicionais no caso da medida de Bairamov e Kotz.

3) Essas medidas herdam as boas propriedades das suas versões globais, não tendo as restrições da necessidade da função densidade conjunta contínua e derivável, não singular no caso da função de dependência local, e a necessidade de a distribuição conjunta ter os segundos momentos finitos no caso da medida de Bairamov e Kotz.

4) Essas medidas não apresentam a debilidade da medida de Bairamov e Kotz de ser uma medida dependente das distribuições marginais envolvidas.

Essas boas propriedades são encorajadoras e o desenvolvimento de mapas de dependência local, baseados nestas medidas, é um campo promissor e interessante para uma pesquisa futura. Mapas onde o quadrado unitário é dividido em "sub-quadrados" menores de mesma área, com as medidas locais propostas sendo calculadas em cada sub-quadrado são uma possibilidade. Além disso, aplicações dessas medidas no cálculo de medidas de risco como o "Conditional Value at Risk" para portfólios com mais de um ativo são uma área de interesse. 


\section{Capítulo 3.}

\section{Estruturas Especiais de Dependência e Limites Maximais de Assimetria}

Neste capítulo serão apresentadas definições e as principais propriedades de duas formas de dependência/simetria que serão tratadas nesta tese, que são: associatividade e bi-simetria.

A importância e as aplicações de cada uma delas também serão discutidas, bem como algumas cópulas especiais que têm relação com essas formas de simetria. A seguir são enunciados os resultados sobre a máxima distância modular que uma cópula pode apresentar em relação aos casos de associatividade e bi-simetria, e serão caracterizadas as famílias de cópulas que atingem essa máxima distância.

\subsection{Definições e Fatos Básicos}

As seguintes cópulas representam papel importante nos resultados desenvolvidos ao longo deste capítulo:

$$
\begin{aligned}
& C_{1}(u, v)=\operatorname{Min}\left(u, v,\left(u-\frac{2}{3}\right)^{+}+\left(v-\frac{1}{3}\right)^{+}\right), \\
& C_{2}(u, v)=\operatorname{Max}\left(0, u+v-1, \frac{1}{3}-\left(\frac{1}{3}-u\right)^{+}+\left(\frac{2}{3}-v\right)^{+}\right),
\end{aligned}
$$

onde $a^{+}=\operatorname{Max}(a, 0)$.

As cópulas $C_{1}$ e $C_{2}$ são casos particulares dos chamados "Shuffles of M" cuja descrição se encontra em Mikusinski et al. (1992), mas que basicamente são cópulas cujos suportes são coleções de segmentos de reta com coeficiente angular +1 ou -1 .

Os suportes de $C_{1}$ e $C_{2}$ são dados na figura abaixo.
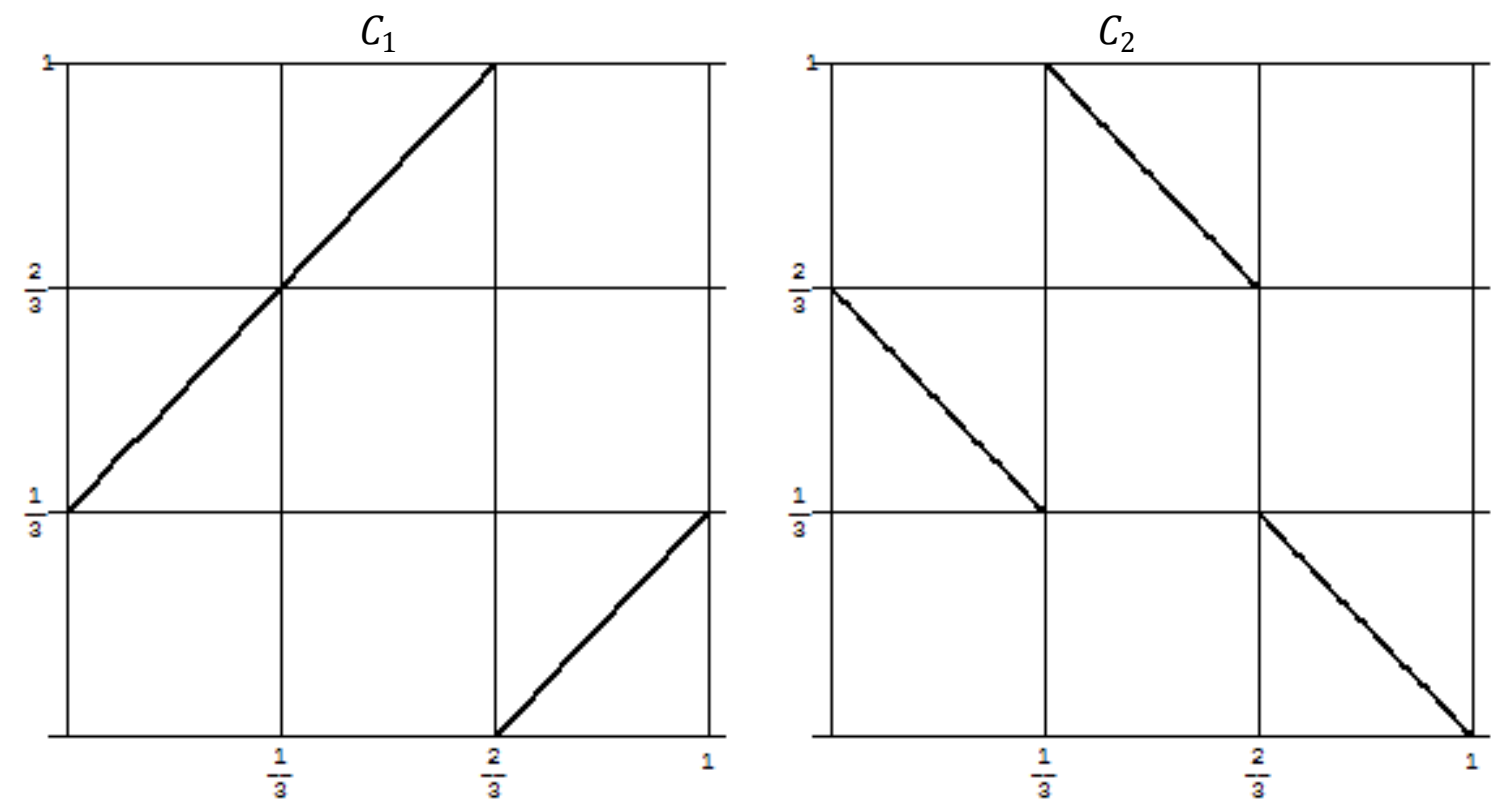

Figura 3.1. Os suportes de $C_{1}$ e $C_{2}$. 
Definição 3.1. Uma cópula bivariada $C(u, v)$ é dita permutável se respeita a seguinte condição:

$$
C(u, v)=C(v, u), \text { para todos } u, v \in[0,1] .
$$

Em Nelsen (2007) e Klement e Mesiar (2006) os autores estudam a distância máxima que uma cópula pode apresentar em relação à permutabilidade segundo a seguinte medida:

$$
\delta_{P}(C)=\operatorname{Sup}_{u, v}|C(u, v)-C(v, u)|, \operatorname{com} u, v \in[0,1]
$$

e o resultado encontrado é que $\delta_{P}(C) \leq \frac{1}{3}$ e que a desigualdade é a melhor possível.

Outros autores estudaram essa medida para cópulas dentro de classes restritas e encontraram diferentes limites, como por exemplo Alvoni e Papini (2007) que obtiveram que $\delta_{P}(C) \leq \frac{1}{5}$ para as cópulas "quasi-concave" lá definidas. Em Durante e Papini (2007), os autores demonstram que $\delta_{P}(C) \leq \frac{1}{4}$ para as cópulas "weak schur concave". Em Baets et al. (2007) os autores provam $\delta_{P}(C) \leq 3-2 \sqrt{2}$ para as cópulas "positive quadrant dependent" e $\delta_{P}(C) \leq \frac{1}{6}$ para as cópulas "vertical semilinear".

O seguinte teorema caracteriza as cópulas que são maximalmente não-permutáveis (aquelas para as quais $\delta_{P}(C)=\frac{1}{3}$ ).

Teorema 3.1. [Nelsen (2007)] Seja $C_{P}$ o conjunto de cópulas bivariadas maximalmente nãopermutáveis e $C$ uma cópula bivariada qualquer. Então:

$$
C \in \mathrm{C}_{\mathrm{P}} \text { se e somente se } C\left(\frac{1}{3}, \frac{2}{3}\right)=\frac{1}{3} \text { e } C\left(\frac{2}{3}, \frac{1}{3}\right)=0 \text { ou } C\left(\frac{1}{3}, \frac{2}{3}\right)=0 \text { e } C\left(\frac{2}{3}, \frac{1}{3}\right)=\frac{1}{3} \text {. }
$$

Definição 3.2. Uma cópula bivariada $C(u, v)$ é dita associativa se respeita a seguinte condição:

$$
C(u, C(v, w))=C(C(u, v), w), \text { para todos } u, v, w \in[0,1] .
$$

A associatividade tem estreita relação com a permutabilidade, conforme indica o Teorema abaixo.

Teorema 3.2. [Alsina et al. (2006)] Seja $T:[0,1]^{2} \rightarrow[0,1]$ um mapeamento contínuo tal que $T(u, 0)=T(0, u)=0$ e $T(u, 1)=T(1, u)=u$ para todo $u \in[0,1]$, e $T(u, T(v, w))=$ $T(T(u, v), w)$ para todos $u, v, w \in[0,1]$. Então $T(u, v)=T(v, u)$, para todos $u, v \in[0,1]$. 
Outra relação importante é entre a associatividade de uma cópula e a sua caracterização Arquimediana (para definições e propriedades das cópulas Arquimedianas, ver Nelsen (2006)), conforme mostra o próximo teorema.

Teorema 3.3. [Ling (1965)] Seja $C$ uma cópula associativa tal que $C(u, u)<u$ para todo $u \in[0,1]$. Então $C$ é Arquimediana.

Um dos resultados obtidos neste capítulo trata de quão não-associativa uma cópula pode ser, o que indiretamente indica o quão distante a cópula está de ter uma caracterização Arquimediana.

Definição 3.3. Uma cópula bivariada $C(u, v)$ é dita bi-simétrica se respeita a seguinte condição:

$$
C(C(u, v), C(w, z))=C(C(u, w), C(v, z)), \text { para todos } u, v, w, z \in[0,1]
$$

A bi-simetria é uma forma de dependência genérica, englobando a permutabilidade $[u, z=1 \Rightarrow C(v, w)=C(w, v)]$ e a associatividade $[w=1 \Rightarrow C(C(u, v), z)=C(u, C(v, z))]$.

A exemplo da não-associatividade, esta capítulo também trata do problema de quão não-bi-simétrica uma cópula pode ser, estendendo os resultados de Nelsen (2007).

\subsection{Limite Máximo de Não-Associatividade}

Nesta seção apresentamos o resultado que caracteriza qual é o limite máximo para a nãoassociatividade de uma cópula bivariada segundo a máxima distância modular e identificamos a classe de cópulas que atinge esse limite.

O limite máximo de não-associatividade é dado no próximo teorema.

Teorema 3.4. Seja $\delta_{A}(C)=\underset{u, v, w}{\operatorname{Sup}}|C(u, C(v, w))-C(C(u, v), w)|$, com $u, v, w \in[0,1]$, a medida de não-associatividade da cópula $C$. Então $\delta_{A}(C) \leq \frac{1}{3}$, qualquer que seja a cópula $C$ e essa desigualdade é a melhor possível.

Prova: Temos, usando propriedades básicas da teoria de cópulas, que as seguintes desigualdades são válidas:

$$
\begin{aligned}
& C(u, C(v, w))-C(C(u, v), w) \leq C(v, w)-C(C(u, v), w) \leq v-C(u, v) \leq 1-u \\
& C(u, C(v, w))-C(C(u, v), w) \leq C(u, w)-C(C(u, v), w) \leq u-C(u, v) \leq 1-v ; \\
& C(u, C(v, w))-C(C(u, v), w) \leq C(u, v)-C(C(u, v), w) \leq 1-w \\
& C(u, C(v, w))-C(C(u, v), w) \leq C(v, w) \leq w \\
& C(u, C(v, w))-C(C(u, v), w) \leq C(u, v) .
\end{aligned}
$$


Portanto

$C(u, C(v, w))-C(C(u, v), w) \leq \operatorname{Min}(u-C(u, v), u-C(u, v), C(u, v)) \leq \operatorname{Min}\left(\frac{u}{2}, \frac{v}{2}\right) \mathrm{e}$ $C(u, C(v, w))-C(C(u, v), w) \leq \operatorname{Min}(1-u, 1-v, 1-w, w)$.

Tem-se então que

$$
C(u, C(v, w))-C(C(u, v), w) \leq \operatorname{Min}\left(1-u, 1-v, 1-w, w, \frac{u}{2}, \frac{v}{2}\right) \leq \frac{1}{3}
$$

Adicionalmente, usando propriedades básicas da teoria de cópulas, temos que as desigualdades abaixo são válidas:

$C(C(u, v), w)-C(u, C(v, w)) \leq C(u, v)-C(u, C(v, w)) \leq v-C(v, w) \leq 1-w ;$

$C(C(u, v), w)-C(u, C(v, w)) \leq C(u, w)-C(u, C(v, w)) \leq w-C(v, w) \leq 1-v$;

$C(C(u, v), w)-C(u, C(v, w)) \leq C(v, w)-C(u, C(v, w)) \leq 1-u$;

$C(C(u, v), w)-C(u, C(v, w)) \leq C(u, v) \leq u$;

$C(C(u, v), w)-C(u, C(v, w)) \leq C(v, w)$.

Portanto

$C(C(u, v), w)-C(u, C(v, w)) \leq \operatorname{Min}(v-C(v, w), w-C(v, w), C(v, w)) \leq \operatorname{Min}\left(\frac{v}{2}, \frac{w}{2}\right) \mathrm{e}$ $C(C(u, v), w)-C(u, C(v, w)) \leq \operatorname{Min}(1-u, 1-v, 1-w, u)$.

Tem-se então que

$$
C(C(u, v), w)-C(u, C(v, w)) \leq \operatorname{Min}\left(1-u, 1-v, 1-w, u, \frac{v}{2}, \frac{w}{2}\right) \leq \frac{1}{3}
$$

Assim obtemos que

$$
|C(u, C(v, w))-C(C(u, v), w)| \leq \frac{1}{3}
$$

Para verificar que a desigualdade é a melhor possível basta notar que

$$
C_{1}\left(\frac{2}{3}, C_{1}\left(\frac{2}{3}, \frac{1}{3}\right)\right)-C_{1}\left(C_{1}\left(\frac{2}{3}, \frac{2}{3}\right), \frac{1}{3}\right)=\frac{1}{3}
$$

No teorema apresentado a seguir é caracterizada a família de cópulas maximalmente não-associativas. 
Teorema 3.5. Seja a medida $\delta_{A}(C)$ como definida acima e sejam $\mathrm{C}_{\mathrm{A}}$ o conjunto de cópulas para as quais $\delta_{A}(C)=\frac{1}{3}$ e $C_{P}$ o conjunto de cópulas para as quais $\delta_{P}(C)=\frac{1}{3}$ (cópulas maximalmente não-permutáveis). Então $\mathrm{C}_{\mathrm{A}}=\mathrm{C}_{\mathrm{P}}$, isto é,

$C \in \mathrm{C}_{\mathrm{A}}\left(\mathrm{C}_{\mathrm{P}}\right)$ se e somente se $C\left(\frac{1}{3}, \frac{2}{3}\right)=\frac{1}{3}$ e $C\left(\frac{2}{3}, \frac{1}{3}\right)=0$ ou $C\left(\frac{1}{3}, \frac{2}{3}\right)=0$ e $C\left(\frac{2}{3}, \frac{1}{3}\right)=\frac{1}{3}$.

Prova: As raízes para $\operatorname{Min}\left(1-u, 1-v, 1-w, w, \frac{u}{2}, \frac{v}{2}\right)=\frac{1}{3}$ são:

$$
\left\{u=\frac{2}{3}, v=\frac{2}{3}, w \in\left[\frac{1}{3}, \frac{2}{3}\right]\right\} .
$$

Além disso, as raízes para $\operatorname{Min}\left(1-u, 1-v, 1-w, u, \frac{v}{2}, \frac{w}{2}\right)=\frac{1}{3}$ são:

$$
\left\{v=\frac{2}{3}, w=\frac{2}{3}, u \in\left[\frac{1}{3}, \frac{2}{3}\right]\right\}
$$

Analisando o caso onde $\operatorname{Min}\left(1-u, 1-v, 1-w, w, \frac{u}{2}, \frac{v}{2}\right)=\frac{1}{3}$ temos que

$$
A_{1}-A_{2}=C\left(\frac{2}{3}, C\left(\frac{2}{3}, w\right)\right)-C\left(C\left(\frac{2}{3}, \frac{2}{3}\right), w\right)=\frac{1}{3}
$$

onde

$A_{1}=C\left(\frac{2}{3}, C\left(\frac{2}{3}, w\right)\right)-C\left(C\left(\frac{2}{3}, \frac{2}{3}\right), C\left(\frac{2}{3}, w\right)\right) \geq 0 \mathrm{e}$

$A_{2}=C\left(C\left(\frac{2}{3}, \frac{2}{3}\right), w\right)-C\left(C\left(\frac{2}{3}, \frac{2}{3}\right), C\left(\frac{2}{3}, w\right)\right) \geq 0$.

Mas $A_{1} \leq \operatorname{Min}\left(\frac{2}{3}-C\left(\frac{2}{3}, \frac{2}{3}\right), C\left(\frac{2}{3}, w\right)\right) \leq \frac{1}{3}$ e portanto $A_{1}=\frac{1}{3}$ e $A_{2}=0$.

Temos então que

$$
\begin{aligned}
& A_{1}=\frac{1}{3} \Rightarrow C\left(\frac{2}{3}, \frac{2}{3}\right)=\frac{1}{3} \text { e } C\left(\frac{2}{3}, w\right) \geq \frac{1}{3} \Rightarrow C\left(\frac{2}{3}, w\right)=\frac{1}{3} \mathrm{e} \\
& A_{2}=0 \Rightarrow C\left(\frac{1}{3}, w\right)=C\left(\frac{1}{3}, \frac{1}{3}\right) .
\end{aligned}
$$

Assim temos que $C\left(\frac{2}{3}, \frac{1}{3}\right)=\frac{1}{3}+C\left(\frac{1}{3}, \frac{1}{3}\right)$.

Como $C\left(\frac{2}{3}, \frac{1}{3}\right) \leq \frac{1}{3}$ e $C\left(\frac{1}{3}, \frac{1}{3}\right) \geq 0$ temos que $C\left(\frac{1}{3}, \frac{1}{3}\right)=0$. 
Dessa forma $C$ deve ser tal que $C\left(\frac{2}{3}, \frac{1}{3}\right)=\frac{1}{3}$ e $C\left(\frac{1}{3}, \frac{2}{3}\right)=0$.

Analisando agora o caso onde $\operatorname{Min}\left(1-u, 1-v, 1-w, u, \frac{v}{2}, \frac{w}{2}\right)=\frac{1}{3}$ temos que

$$
A_{1}-A_{2}=C\left(C\left(u, \frac{2}{3}\right), \frac{2}{3}\right)-C\left(u, C\left(\frac{2}{3}, \frac{2}{3}\right)\right)=\frac{1}{3}
$$

onde

$$
\begin{aligned}
& A_{1}=C\left(C\left(u, \frac{2}{3}\right), \frac{2}{3}\right)-C\left(C\left(u, \frac{2}{3}\right), C\left(\frac{2}{3}, \frac{2}{3}\right)\right) \mathrm{e} \\
& A_{2}=C\left(u, C\left(\frac{2}{3}, \frac{2}{3}\right)\right)-C\left(C\left(u, \frac{2}{3}\right), C\left(\frac{2}{3}, \frac{2}{3}\right)\right) .
\end{aligned}
$$

Mas $A_{1} \leq \operatorname{Min}\left(\frac{2}{3}-C\left(\frac{2}{3}, \frac{2}{3}\right), C\left(u, \frac{2}{3}\right)\right) \leq \frac{1}{3}$ e portanto $A_{1}=\frac{1}{3}$ e $A_{2}=0$.

Temos então que

$A_{1}=\frac{1}{3} \Rightarrow C\left(\frac{2}{3}, \frac{2}{3}\right)=\frac{1}{3}$ e $C\left(u, \frac{2}{3}\right) \geq \frac{1}{3} \Rightarrow C\left(u, \frac{2}{3}\right)=\frac{1}{3} \mathrm{e}$

$A_{2}=0 \Rightarrow C\left(u, \frac{1}{3}\right)=C\left(\frac{1}{3}, \frac{1}{3}\right) \Rightarrow C\left(\frac{2}{3}, \frac{1}{3}\right)=C\left(\frac{1}{3}, \frac{1}{3}\right)$

Como $A_{1}=\frac{1}{3}$ temos que $C\left(\frac{1}{3}, \frac{2}{3}\right)=\frac{1}{3}+C\left(\frac{1}{3}, \frac{1}{3}\right)$.

Como $C\left(\frac{1}{3}, \frac{2}{3}\right) \leq \frac{1}{3}$ e $C\left(\frac{1}{3}, \frac{1}{3}\right) \geq 0$ temos que $C\left(\frac{1}{3}, \frac{1}{3}\right)=0$.

Dessa forma $C$ deve ser tal que $C\left(\frac{2}{3}, \frac{1}{3}\right)=0$ e $C\left(\frac{1}{3}, \frac{2}{3}\right)=\frac{1}{3}$.

Portanto $C \in \mathrm{C}_{\mathrm{A}}$ se e somente se $C\left(\frac{1}{3}, \frac{2}{3}\right)=\frac{1}{3}$ e $C\left(\frac{2}{3}, \frac{1}{3}\right)=0$ ou $C\left(\frac{1}{3}, \frac{2}{3}\right)=0$ e $C\left(\frac{2}{3}, \frac{1}{3}\right)=\frac{1}{3}$.

Dessa forma temos que, à semelhança do caso de não-permutabilidade, a medida de não-associatividade baseada na máxima distância modular tem $\frac{1}{3}$ como valor máximo, e que as cópulas que são maximalmente não-permutáveis, segundo a medida definida em Nelsen (2007), são exatamente aquelas que são maximalmente não-associativas, segundo a medida definida anteriormente, e vice-versa. 


\subsection{Limite Máximo de Não-Bi-Simetria}

Nesta seção apresentamos o resultado que caracteriza qual é o limite máximo de não-bisimetria de uma cópula bivariada segundo a máxima distância modular e identificamos a classe de cópulas que atinge esse limite.

O limite máximo de não-bi-simetria é dado no próximo teorema.

Teorema 3.6. Seja $\delta_{B}(C)=\operatorname{Sup}_{u, v, w, z}|C(C(u, v), C(w, z))-C(C(u, w), C(v, z))|$, com $u, v, w, z \in[0,1]$, a medida de não-bi-simetria da cópula $C$. Então $\delta_{B}(C) \leq \frac{1}{3}$, qualquer que seja a cópula $C$ e essa desigualdade é a melhor possível.

Prova: Temos, usando propriedades básicas da teoria de cópulas, que as seguintes desigualdades são válidas:

$C(C(u, v), C(w, z))-C(C(u, w), C(v, z)) \leq w-C(u, w)+z-C(v, z)$;

$C(C(u, v), C(w, z))-C(C(u, w), C(v, z)) \leq C(u, w)$;

$C(C(u, v), C(w, z))-C(C(u, w), C(v, z)) \leq C(v, z)$.

\section{Portanto}

$$
\begin{aligned}
& C(C(u, v), C(w, z))-C(C(u, w), C(v, z)) \\
& \quad \leq \operatorname{Min}\left(\frac{w+z-C(u, w)}{2}, \frac{w+z-C(v, z)}{2}\right) \leq \operatorname{Min}\left(\frac{1-u+z}{2}, \frac{1+w-v}{2}\right) .
\end{aligned}
$$

Adicionalmente

$$
C(C(u, v), C(w, z))-C(C(u, w), C(v, z)) \leq u-C(u, w)+v-C(v, z)
$$

Portanto

$$
\begin{aligned}
& C(C(u, v), C(w, z))-C(C(u, w), C(v, z)) \\
& \quad \leq \operatorname{Min}\left(\frac{u+v-C(u, w)}{2}, \frac{u+v-C(v, z)}{2}\right) \leq \operatorname{Min}\left(\frac{1-w+v}{2}, \frac{1-z+u}{2}\right) .
\end{aligned}
$$

Além disso, temos que

$$
\begin{aligned}
& C(C(u, v), C(w, z))-C(C(u, w), C(v, z)) \\
& \leq \operatorname{Max}(|C(u, v)-C(u, w)|,|C(w, z)-C(v, z)|) \leq|v-w|
\end{aligned}
$$


Dessa forma temos que

$$
\begin{aligned}
& C(C(u, v), C(w, z))-C(C(u, w), C(v, z)) \\
& \quad \leq \operatorname{Min}\left(u, v, w, z,|v-w|, \frac{1-w+v}{2}, \frac{1-z+u}{2}, \frac{1-u+z}{2}, \frac{1+w-v}{2}\right) \leq \frac{1}{3} .
\end{aligned}
$$

Adicionalmente, usando propriedades básicas da teoria de cópulas, temos que as desigualdades abaixo são válidas:

$C(C(u, w), C(v, z))-C(C(u, v), C(w, z)) \leq u-C(u, v)+w-C(w, z) ;$

$C(C(u, w), C(v, z))-C(C(u, v), C(w, z)) \leq C(u, v)$

$C(C(u, w), C(v, z))-C(C(u, v), C(w, z)) \leq C(w, z)$.

\section{Portanto}

$$
\begin{aligned}
& C(C(u, w), C(v, z))-C(C(u, v), C(w, z)) \\
& \leq \operatorname{Min}\left(\frac{u+w-C(w, z)}{2}, \frac{u+w-C(u, v)}{2}\right) \leq \operatorname{Min}\left(\frac{1-z+u}{2}, \frac{1-v+w}{2}\right) .
\end{aligned}
$$

Temos também que

$C(C(u, w), C(v, z))-C(C(u, v), C(w, z)) \leq v-C(u, v)+z-C(w, z)$.

\section{Portanto}

$$
\begin{aligned}
& C(C(u, w), C(v, z))-C(C(u, v), C(w, z)) \\
& \leq \operatorname{Min}\left(\frac{z+v-C(u, v)}{2}, \frac{z+v-C(w, z)}{2}\right) \leq \operatorname{Min}\left(\frac{1-u+z}{2}, \frac{1-w+v}{2}\right) .
\end{aligned}
$$

Além disso temos que

$$
\begin{aligned}
& C(C(u, w), C(v, z))-C(C(u, v), C(w, z)) \\
& \leq \operatorname{Max}(|C(u, w)-C(u, v)|,|C(v, z)-C(w, z)|) \leq|v-w| .
\end{aligned}
$$

Dessa forma temos que

$$
\begin{aligned}
& C(C(u, w), C(v, z))-C(C(u, v), C(w, z)) \\
& \quad \leq \operatorname{Min}\left(u, v, w, z,|v-w|, \frac{1-w+v}{2}, \frac{1-z+u}{2}, \frac{1-u+z}{2}, \frac{1+w-v}{2}\right) \leq \frac{1}{3} .
\end{aligned}
$$

Assim obtemos que

$$
|C(C(u, v), C(w, z))-C(C(u, w), C(v, z))| \leq \frac{1}{3}
$$


Para verificar que a desigualdade é a melhor possível basta notar que

$$
C_{1}\left(C_{1}\left(1, \frac{2}{3}\right), C_{1}\left(\frac{1}{3}, 1\right)\right)-C_{1}\left(C_{1}\left(1, \frac{1}{3}\right), C_{1}\left(\frac{2}{3}, 1\right)\right)=\frac{1}{3}
$$

A família de cópulas que atingem o limite máximo de não-bi-simetria é dada no próximo teorema.

Teorema 3.7. Seja a medida $\delta_{B}(C)$ como definida acima e sejam $\mathrm{C}_{\mathrm{B}}$ o conjunto de cópulas para as quais $\delta_{B}(C)=\frac{1}{3}$ e $\mathrm{C}_{\mathrm{P}}$ o conjunto de cópulas para as quais $\delta_{P}(C)=\frac{1}{3}$ (cópulas maximalmente não-permutáveis). Então $C_{B}=C_{P}$, isto é,

$C \in \mathrm{C}_{\mathrm{B}}\left(\mathrm{C}_{\mathrm{P}}\right)$ se e somente se ou $C\left(\frac{1}{3}, \frac{2}{3}\right)=\frac{1}{3}$ e $C\left(\frac{2}{3}, \frac{1}{3}\right)=0$ ou $C\left(\frac{1}{3}, \frac{2}{3}\right)=0$ e $C\left(\frac{2}{3}, \frac{1}{3}\right)=\frac{1}{3}$.

Prova: As raízes de $\operatorname{Min}\left(u, v, w, z,|v-w|, \frac{1-w+v}{2}, \frac{1-z+u}{2}, \frac{1-u+z}{2}, \frac{1+w-v}{2}\right)=\frac{1}{3}$ são

$$
\left\{u \geq \frac{1}{3}, v \geq \frac{1}{3}, w \geq \frac{1}{3}, z \geq \frac{1}{3},|u-z| \leq \frac{1}{3},|v-w|=\frac{1}{3}\right\}
$$

Analisando $C(C(u, v), C(w, z))-C(C(u, w), C(v, z))=\frac{1}{3}$, precisamos considerar os seguintes casos:

1. Seja $w>v$.

Temos que

onde

$$
A_{1}-A_{2}=C(C(u, v), C(w, z))-C(C(u, w), C(v, z))=\frac{1}{3}
$$

$A_{1}=C(C(u, v), C(w, z))-C(C(u, v), C(v, z)) \geq 0 \mathrm{e}$

$A_{2}=C(C(u, w), C(v, z))-C(C(u, v), C(v, z)) \geq 0$.

Como $w>v$ e $|v-w|=\frac{1}{3}$, temos que $w=v+\frac{1}{3}$.

Mas $A_{1} \leq \operatorname{Min}\left(C\left(v+\frac{1}{3}, z\right)-C(v, z), C(u, v)\right) \leq \frac{1}{3}$ e portanto $A_{1}=\frac{1}{3}$ e $A_{2}=0$.

Temos então que

$$
A_{1}=\frac{1}{3} \Rightarrow C\left(v+\frac{1}{3}, z\right)-C(v, z)=\frac{1}{3} \text { e } C(u, v) \geq \frac{1}{3} .
$$


Tomando-se $(v, z)=\left(\frac{1}{3}, \frac{1}{3}\right)$, chega-se a $C\left(\frac{2}{3}, \frac{1}{3}\right)=\frac{1}{3}+C\left(\frac{1}{3}, \frac{1}{3}\right)$.

Como $C\left(\frac{2}{3}, \frac{1}{3}\right) \leq \frac{1}{3}$ e $C\left(\frac{1}{3}, \frac{1}{3}\right) \geq 0$, temos que $C\left(\frac{1}{3}, \frac{1}{3}\right)=0$.

Tomando-se $(v, z)=\left(\frac{2}{3}, \frac{2}{3}\right)$, chega-se a $C\left(1, \frac{2}{3}\right)=\frac{1}{3}+C\left(\frac{2}{3}, \frac{2}{3}\right) \Rightarrow C\left(\frac{2}{3}, \frac{2}{3}\right)=\frac{1}{3}$.

Dessa forma $C$ deve ser tal que $C\left(\frac{2}{3}, \frac{1}{3}\right)=\frac{1}{3}$ e $C\left(\frac{1}{3}, \frac{2}{3}\right)=0$.

2. Seja $v>w$.

Temos que

onde

$$
A_{1}-A_{2}=C(C(u, v), C(w, z))-C(C(u, w), C(v, z))=\frac{1}{3}
$$

$A_{1}=C(C(u, v), C(w, z))-C(C(u, w), C(w, z)) \geq 0 \mathrm{e}$

$A_{2}=C(C(u, w), C(v, z))-C(C(u, w), C(w, z)) \geq 0$.

Como $v>w$ e $|v-w|=\frac{1}{3}$, temos que $v=w+\frac{1}{3}$.

Mas $A_{1} \leq \operatorname{Min}\left(C\left(u, w+\frac{1}{3}\right)-C(u, w), C(w, z)\right) \leq \frac{1}{3}$ e portanto $A_{1}=\frac{1}{3}$ e $A_{2}=0$.

Temos então que

$$
A_{1}=\frac{1}{3} \Rightarrow C\left(u, w+\frac{1}{3}\right)-C(u, w)=\frac{1}{3} \text { e } C(w, z) \geq \frac{1}{3} .
$$

Tomando-se $(u, w)=\left(\frac{1}{3}, \frac{1}{3}\right)$, chega-se a $C\left(\frac{1}{3}, \frac{2}{3}\right)=\frac{1}{3}+C\left(\frac{1}{3}, \frac{1}{3}\right)$.

Como $C\left(\frac{1}{3}, \frac{2}{3}\right) \leq \frac{1}{3}$ e $C\left(\frac{1}{3}, \frac{1}{3}\right) \geq 0$, temos que $C\left(\frac{1}{3}, \frac{1}{3}\right)=0$.

Tomando-se $(v, z)=\left(\frac{2}{3}, \frac{2}{3}\right)$, chega-se a $C\left(\frac{2}{3}, 1\right)=\frac{1}{3}+C\left(\frac{2}{3}, \frac{2}{3}\right) \Rightarrow C\left(\frac{2}{3}, \frac{2}{3}\right)=\frac{1}{3}$.

Dessa forma $C$ deve ser tal que $C\left(\frac{2}{3}, \frac{1}{3}\right)=0$ e $C\left(\frac{1}{3}, \frac{2}{3}\right)=\frac{1}{3}$.

Analisando $C(C(u, w), C(v, z))-C(C(u, v), C(w, z))=\frac{1}{3}$ precisamos considerar os seguintes casos 


\section{Seja $w>v$.}

Temos que

onde

$$
A_{1}-A_{2}=C(C(u, w), C(v, z))-C(C(u, v), C(w, z))=\frac{1}{3}
$$

$A_{1}=C(C(u, w), C(v, z))-C(C(u, v), C(v, z)) \geq 0 \mathrm{e}$

$A_{2}=C(C(u, v), C(w, z))-C(C(u, v), C(v, z)) \geq 0$.

Como $w>v$ e $|v-w|=\frac{1}{3}$, temos que $w=v+\frac{1}{3}$.

Mas $A_{1} \leq \operatorname{Min}\left(C\left(u, v+\frac{1}{3}\right)-C(u, v), C(v, z)\right) \leq \frac{1}{3}$ e portanto $A_{1}=\frac{1}{3}$ e $A_{2}=0$.

Temos então que

$$
A_{1}=\frac{1}{3} \Rightarrow C\left(u, v+\frac{1}{3}\right)-C(u, v)=\frac{1}{3} \text { e } C(v, z) \geq \frac{1}{3} .
$$

Tomando-se $(u, v)=\left(\frac{1}{3}, \frac{1}{3}\right)$, chega-se a $C\left(\frac{1}{3}, \frac{2}{3}\right)=\frac{1}{3}+C\left(\frac{1}{3}, \frac{1}{3}\right)$.

Como $C\left(\frac{1}{3}, \frac{2}{3}\right) \leq \frac{1}{3}$ e $C\left(\frac{1}{3}, \frac{1}{3}\right) \geq 0$, temos que $C\left(\frac{1}{3}, \frac{1}{3}\right)=0$.

Tomando-se $(u, v)=\left(\frac{2}{3}, \frac{2}{3}\right)$, chega-se a $C\left(\frac{2}{3}, 1\right)=\frac{1}{3}+C\left(\frac{2}{3}, \frac{2}{3}\right) \Rightarrow C\left(\frac{2}{3}, \frac{2}{3}\right)=\frac{1}{3}$.

Dessa forma $C$ deve ser tal que $C\left(\frac{2}{3}, \frac{1}{3}\right)=0$ e $C\left(\frac{1}{3}, \frac{2}{3}\right)=\frac{1}{3}$.

4. Seja $v>w$.

Temos que

onde

$$
A_{1}-A_{2}=C(C(u, w), C(v, z))-C(C(u, v), C(w, z))=\frac{1}{3},
$$

$A_{1}=C(C(u, w), C(v, z))-C(C(u, w), C(w, z)) \geq 0 \mathrm{e}$

$A_{2}=C(C(u, v), C(w, z))-C(C(u, w), C(w, z)) \geq 0$.

Como $v>w$ e $|v-w|=\frac{1}{3}$, temos que $v=w+\frac{1}{3}$. 
Mas $A_{1} \leq \operatorname{Min}\left(C\left(w+\frac{1}{3}, z\right)-C(w, z), C(u, w)\right) \leq \frac{1}{3}$ e portanto $A_{1}=\frac{1}{3}$ e $A_{2}=0$.

Temos então que

$$
A_{1}=\frac{1}{3} \Rightarrow C\left(w+\frac{1}{3}, z\right)-C(w, z)=\frac{1}{3} \text { e } C(u, w) \geq \frac{1}{3}
$$

Tomando-se $(w, z)=\left(\frac{1}{3}, \frac{1}{3}\right)$, chega-se a $C\left(\frac{2}{3}, \frac{1}{3}\right)=\frac{1}{3}+C\left(\frac{1}{3}, \frac{1}{3}\right)$.

Como $C\left(\frac{2}{3}, \frac{1}{3}\right) \leq \frac{1}{3}$ e $C\left(\frac{1}{3}, \frac{1}{3}\right) \geq 0$, temos que $C\left(\frac{1}{3}, \frac{1}{3}\right)=0$.

Tomando-se $(w, z)=\left(\frac{2}{3}, \frac{2}{3}\right)$, chega-se a $C\left(1, \frac{2}{3}\right)=\frac{1}{3}+C\left(\frac{2}{3}, \frac{2}{3}\right) \Rightarrow C\left(\frac{2}{3}, \frac{2}{3}\right)=\frac{1}{3}$.

Dessa forma $C$ deve ser tal que $C\left(\frac{2}{3}, \frac{1}{3}\right)=0$ e $C\left(\frac{1}{3}, \frac{2}{3}\right)=\frac{1}{3}$.

Portanto $C \in \mathrm{C}_{\mathrm{B}}$ se e somente se $C\left(\frac{1}{3}, \frac{2}{3}\right)=\frac{1}{3}$ e $C\left(\frac{2}{3}, \frac{1}{3}\right)=0$ ou $C\left(\frac{1}{3}, \frac{2}{3}\right)=0$ e $C\left(\frac{2}{3}, \frac{1}{3}\right)=\frac{1}{3}$.

Dessa forma temos que, à semelhança dos casos de não-permutabilidade e não-

associatividade, a medida de não-bi-simetria baseada na máxima distância modular tem $\frac{1}{3}$ como valor máximo. Adicionalmente temos que a família de cópulas que são maximalmente nãopermutáveis (e também maximalmente não-associativas) é exatamente aquela onde as cópulas são maximalmente não-bi-simétricas. Tem-se então que são válidos os seguintes resultados.

Teorema 3.8. [Nelsen (2007)] Seja C o conjunto de cópulas bivariadas maximalmente assimétricas, segundo os critérios de não-permutabilidade, não-associatividade e não-bisimetria, e $C$ uma cópula bivariada qualquer. Então:

(i) $\quad C$ é tal que $C\left(\frac{1}{3}, \frac{2}{3}\right)=\frac{1}{3}$ e $C\left(\frac{2}{3}, \frac{1}{3}\right)=0$ se e somente se $C_{1} \prec C \prec C_{2}$ e é tal que $C\left(\frac{1}{3}, \frac{2}{3}\right)=0$ e $C\left(\frac{2}{3}, \frac{1}{3}\right)=\frac{1}{3}$ se e somente se $C_{1}^{T} \prec C \prec C_{2}^{T}$, onde $C^{T}(u, v)=C(v, u)$;

(ii) Toda cópula maximalmente não-permutável (não-associativa e não-bi-simétrica) tem a mesma seção diagonal, isto é, para toda cópula $C \in \mathrm{C}, C(u, u)=\left(u-\frac{1}{3}\right)^{+}+\left(u-\frac{2}{3}\right)^{+}$;

(iii) Para toda cópula $C \in \mathrm{C}$ temos que $\tau_{C} \in\left[\frac{-5}{9}, \frac{-1}{3}\right]$ e $\rho_{C} \in\left[\frac{-5}{9}, \frac{1}{9}\right]$.

O que fica evidenciado, portanto, é a estreita ligação entre os conceitos de permutabilidade, associatividade e bi-simetria, especialmente quando se trata das cópulas mais distantes de terem essas propriedades segundo os critérios definidos ao longo do texto, uma que são as mesmas em todos os casos. 


\section{Capítulo 4.}

\section{Medidas e Testes de Hipóteses de Assimetria de Distribuições Bivariadas}

Para entender o fenômeno de assimetria, apresentaremos alguns conceitos de simetria de uma distribuição bivariada. Para uma exposição mais detalhada sobre definições alternativas e suas propriedades ver Snijders (1981) e Yanagimoto e Sibuya (1971).

Neste capítulo serão tratadas a não-permutabilidade e a assimetria radial. Suas definições e as aplicações destes conceitos serão apresentadas ao longo do capítulo, conforme forem necessários para os desenvolvimentos. O objetivo é propor medidas e testes de hipóteses que identifiquem a presença dessas modalidades de assimetria nos dados.

\subsection{Principais Conceitos de Permutabilidade}

Um vetor aleatório bivariado $(X, Y)$ é dito permutável se é identicamente distribuído ao vetor $(Y, X)$. Conseqüentemente, se a função distribuição conjunta de $X$ e $Y$ é $F(x, y)$, então $F(x, y)=F(y, x)$ para todo $(x, y) \in \overline{\mathfrak{R}}^{2}$.

A igualdade das funções distribuição conjuntas implica que as funções marginais das variáveis $X$ e $Y$ são idênticas. Adicionalmente, se o vetor aleatório $(X, Y)$ é contínuo e tem uma cópula subjacente $C(u, v)$, então é válido o seguinte resultado:

Teorema 4.1. [Nelsen (2006)] Sejam $X$ e $Y$ variáveis aleatórias contínuas com distribuição conjunta $H(x, y)$, marginais $F$ e $G$, respectivamente, e cópula $C(u, v)$. Então $X$ e $Y$ são permutáveis se e somente se $F=G$ e $C(u, v)=C(v, u)$, para todo $(u, v)$ em $[0,1]^{2}$.

A tabela 4.1 abaixo é dada em Bell e Haller (1969). Nesta tabela são apresentadas formulações de permutabilidade para os casos: contínuo, absolutamente contínuo e distribuição Normal bivariada.

Tabela 4.1. [Bell e Haller (1969)]. Formulações de Permutabilidade

\begin{tabular}{llll}
\hline & Contínuo & Absolutamente Contínuo & \multicolumn{1}{c}{ Normal bivariada } \\
\hline (A) $\quad \begin{array}{l}F(x, y)=F(y, x), \\
\text { para todos } x, y .\end{array}$ & $\begin{array}{l}f(x, y)=f(y, x), \\
\text { para todos } x, y .\end{array}$ & $\begin{array}{l}E(X)=E(Y) \mathrm{e} \\
\operatorname{Var}(X)=\operatorname{Var}(Y) .\end{array}$ \\
(B) $\quad \begin{array}{l}2 G(w, 0)=G(w, v)+G(w,-v), \\
\text { para todos } w, v .\end{array}$ & $\begin{array}{l}g(w, v)=g(w,-v), \\
\text { para todos } w, v .\end{array}$ & $\begin{array}{l}E(V)=0 \mathrm{e} \\
\operatorname{Cov}(W, V)=0 .\end{array}$ \\
(C) $\quad \begin{array}{l}G_{V \mid W}(v \mid w)+G_{V \mid W}(-v \mid w)=1, \\
\text { para todos } w, v .\end{array}$ & $\begin{array}{l}g(v, w)=g(-v, w), \\
\text { para todos } w, v .\end{array}$ & $\begin{array}{l}E(V \mid W=w)=0, \\
\text { para todo } w .\end{array}$ \\
\hline
\end{tabular}




\begin{tabular}{llll}
\hline (D) & $\begin{array}{l}F_{X}=F_{Y}, \text { i.e. as marginais de } X \text { e } \\
Y \text { são iguais. }\end{array}$ & $\begin{array}{l}f_{X}=f_{Y} \text {, i.e. as densidades } \\
\text { de } X \text { e } Y \text { são iguais. }\end{array}$ & $\begin{array}{l}E(X)=E(Y) \mathrm{e} \\
\operatorname{Var}(X)=\operatorname{Var}(Y) .\end{array}$ \\
(E) $\quad \begin{array}{l}G_{V}(t)+G_{V}(-t)=1, \\
\text { para todo } t .\end{array}$ & $\begin{array}{l}g_{V}(t)=g_{V}(-t), \\
\text { para todo } t .\end{array}$ & $E(V)=0$. \\
(F) $\quad P(X>Y)=\frac{1}{2}=G_{V}(0)$. & $\int_{-\infty}^{0} g_{V}(t) d t=\frac{1}{2}$. & $E(V)=0$. \\
(G) $\quad \begin{array}{l}G_{V \mid W}(0 \mid w)=\frac{1}{2}, \\
\text { para todo } w .\end{array}$ & $\begin{array}{l}\int_{-\infty}^{0} g_{V \mid W}(v \mid w) d v=\frac{1}{2}, \\
\text { para todo } w .\end{array}$ & $\begin{array}{l}E(V \mid W=w)=0, \\
\text { para todo } w .\end{array}$ \\
\hline
\end{tabular}

Notações: $(X, Y)$ é o vetor aleatório analisado, $(W, V)$ é o vetor aleatório transformado, onde $W=X+Y$ e $V=Y-X$. As funções $F$ e $G$ denotam as distribuições conjuntas de $(X, Y)$ e $(W, V)$, respectivamente. $F_{X}, F_{Y}, G_{V}$ e $G_{w}$ são as marginais e $G_{V \mid W}(v \mid w)$ é a distribuição condicional de $V$ dado $W$. As funções $f, g, f_{X}, f_{Y}, g_{V}$ e $g_{V \mid W}(v \mid w)$ são as densidades correspondentes e $E(),. \operatorname{Var}($.$) e \operatorname{Cov}(.,$.$) representam as funções esperança, variância e$ covariância, respectivamente.

Os dois teoremas seguintes estabelecem as relações entre as condições (A)-(G) dadas acima.

Teorema 4.2. [Bell e Haller (1969)] Para a distribuição normal bivariada

(I) As condições (A), (B), (C), (D) e (G) são equivalentes;

(II) As condições (E) e (F) são equivalentes; $\mathrm{e}$

(III) As condições em (I) implicam aquelas em (II), mas a volta não é válida.

Teorema 4.3. [Bell e Haller (1969)] Para distribuições bivariadas contínuas (e absolutamente contínuas)

(I) As condições (A), (B) e (C) são equivalentes;

(II) Não existem outras relações de equivalência;

(III) As condições (A), (B) e (C) implicam cada uma das demais; e

(IV) A condição (F) é implicada por (G) e também por (C).

Em resumo:

1) No caso normal a permutabilidade é equivalente a $E(V)=0$ e à independência de $W$ e $V$, onde $(W, V)=(X+Y, Y-X)$; e à igualdade das médias e variâncias de $(X, Y)$.

2) No caso contínuo a permutabilidade é equivalente à simetria da distribuição condicional de $V$ para todo valor de $W$. 
O conceito de permutabilidade tem importantes aplicações em muitas áreas da estatística: teoria de valores extremos, processos estocásticos, estatística Bayesiana, etc.

Existem na literatura vários testes para identificação de permutabilidade, ver por exemplo Bell e Haller (1969), Hollander (1971) e Snijders (1981), mas poucos esforços foram feitos no sentido de se criar medidas para avaliar o grau de não-permutabilidade. Uma proposta para tal é feita em Nelsen (2007), que estabelece uma medida baseada no supremo do módulo da distância entre $C(u, v)$ e $C(v, u)$ para todo $(u, v)$ em $[0,1]^{2}$, isto é, $\operatorname{Sup}_{u, v}|C(u, v)-C(v, u)|$, para $(u, v) \in[0,1]^{2}$, conforme mostrado no capítulo 3 .

O seguinte exemplo mostra que esta medida não é informativa quando aplicada a funções distribuição, por exemplo $F(x, y)$.

Exemplo 4.1. [Nelsen (2007)] Sejam os suportes de $X$ e $Y$ subconjuntos de $(0,1)$ e $(1,2)$, respectivamente. Então $F(1,2)=1$ e $F(2,1)=0$, e $\operatorname{Sup}_{x, y}|F(x, y)-F(y, x)|=1$.

Portanto, esta medida tem sentido em termos de cópulas, podendo ser aplicada somente para distribuições bivariadas com marginais idênticas.

\subsubsection{Teste de Hipótese de Permutabilidade}

Um exemplo de teste de permutabilidade é proposto em Ernst e Schucany (1999), onde os autores propõem a seguinte formulação:

Seja o vetor aleatório contínuo $(X, Y)$ com segundos momentos finitos e vetor de médias $\boldsymbol{\mu}=\left(\mu_{X}, \mu_{Y}\right)^{T}$ e matriz de covariâncias dada por

$$
\boldsymbol{\Sigma}=\left[\begin{array}{cc}
\sigma_{X}^{2} & \rho_{X, Y} \sigma_{X} \sigma_{Y} \\
\rho_{X, Y} \sigma_{X} \sigma_{Y} & \sigma_{Y}^{2}
\end{array}\right],
$$

onde $\rho_{X, Y}=\operatorname{Corr}(X, Y), \mu_{X}=E(X), \mu_{Y}=E(Y), \sigma_{X}^{2}=\operatorname{Var}(X)$ e $\sigma_{Y}^{2}=\operatorname{Var}(Y)$.

Então o teste de permutabilidade com nível de significância $\alpha$ é definido por:

$\mathcal{H}_{0}: F(x, y)=F(y, x)$, para todo $(x, y)$ em $\bar{\Re}^{2}$,

contra

$\mathcal{H}_{1}: \mu_{X} \neq \mu_{Y}$ ou $\sigma_{X}^{2} \neq \sigma_{Y}^{2}$.

A hipótese alternativa se baseia no fato que a permutabilidade implica na igualdade das distribuições marginais de $X$ e $Y$. Devido a essa definição, esse teste falha em apontar situações em que as distribuições marginais são idênticas, mas sua estrutura de dependência é nãopermutável. Para maiores detalhes sobre a estatística do teste e sua distribuição, ver Ernst e Schucany (1999). 


\subsubsection{Aplicações de Permutabilidade em Finanças}

Uma aplicação dos conceitos e testes de permutabilidade em finanças é feito em Klöessner (2007), onde o autor trata da suposição de que retornos intra-diários sejam simétricos ou que sejam um processo de Lévy para os índices de ações Dow-Jones, S\&P 500 e DAX e suas ações componentes. A metodologia é descrita a seguir:

Sejam $\left(P_{t}\right)_{t \in[0,1]}$ os preços de um ativo em um dia de negociação, onde se assume que os negócios sejam realizados no intervalo $[0,1]$. Sejam também $X_{t}:=\log P_{t}$ os log-preços e $r_{t}:=X_{t}-X_{0}$ os log-retornos. Usando essas notações, os retornos maximais, minimais e diários são definidos por

$$
r_{\max }:=\operatorname{Sup}_{t \in[0,1]} r_{t}, \quad r_{\min }:=\operatorname{Inf}_{t \in[0,1]} r_{t}, \quad r_{d i a}:=r_{1}
$$

A variável $r_{t}$ representa o log-retorno que é obtido comprando-se o ativo na abertura dos negócios $(t=0)$ e vendendo-se no instante $t$. Pela definição temos que $r_{\max } \geq 0 \mathrm{e}$ $r_{\min } \leq 0$. As seguintes relações são válidas:

$$
\begin{aligned}
& r_{\text {max }}=\log \left(\operatorname{Sup}_{t \in[0,1]} P_{t}\right)-\log \left(P_{0}\right) \\
& r_{\text {min }}=\log \left(\operatorname{Inf}_{t \in[0,1]} P_{t}\right)-\log \left(P_{0}\right) \\
& r_{d i a}=\log \left(P_{1}\right)-\log \left(P_{0}\right)
\end{aligned}
$$

Sejam as seguintes medidas de volatilidades intra-diárias de alta e baixa definidas por

$$
V_{\text {max }}:=2 r_{\max }\left(r_{\max }-r_{\text {dia }}\right) \text { e } V_{\min }:=2 r_{\min }\left(r_{\min }-r_{\text {dia }}\right)
$$

No caso onde $X_{t}$ é o Movimento Browniano com tendência $\mu$ e volatilidade $\sigma^{2}$, temos que $V_{\max }$ e $V_{\min }$ são distribuídas exponencialmente com parâmetro $\frac{1}{\sigma^{2}}$.

Sejam os seguintes índices de volatilidade intra-diárias de alta e baixa definidos por

$$
R_{\max }:=\frac{V_{\max }}{\left[r_{d i a}-E\left(r_{\text {dia }}\right)\right]^{2}} \text { e } R_{\text {min }}:=\frac{V_{\text {min }}}{\left[r_{d i a}-E\left(r_{d i a}\right)\right]^{2}}
$$

No caso onde $X_{t}$ é o Movimento Browniano, temos que $R_{\max }$ e $R_{\min }$ são distribuídos como uma distribuição $F$ de Snedocor de parâmetros 2 e 1, isto é, $F(2,1)$, o que implica que não existem nem média nem momentos de ordem superior para essas variáveis. 
Para contornar esse problema são feitas as seguintes transformações monótonas e bijetivas, definidas por

$$
U_{\max }:=1-\frac{1}{\sqrt{1+2 R_{\max }}} \text { e } U_{\min }:=1-\frac{1}{\sqrt{1+2 R_{\min }}} .
$$

No caso onde $X_{t}$ é o Movimento Browniano, temos que $U_{\max }$ e $U_{\min }$ são uniformes no intervalo $(0,1)$.

As medidas $V_{\text {max }}, V_{\text {min }}, R_{\text {max }}, R_{\text {min }}, U_{\max }$ e $U_{\min }$ possuem algumas propriedades de simetria quando o processo $\left(X_{t}\right)_{t \geq 0}$ de retornos é um processo de Lévy, o qual é definido a seguir.

Definição 4.1. Um processo estocástico $\left(X_{t}\right)_{t \geq 0} \operatorname{com} X_{0}=0$ é chamado um processo de Lévy se

1. Para toda seqüência crescente de instantes $t_{0}, \ldots, t_{n}$, as variáveis aleatórias $X_{t_{0}}, X_{t_{1}}-X_{t_{0}}, \ldots, X_{t_{n}}-X_{t_{n-1}}$ são independentes (incrementos independentes);

2. A distribuição de $X_{t+h}-X_{t}$ não depende de $\mathrm{t}$ (incrementos estacionários);

3. $\lim _{h \rightarrow 0} P\left(\left|X_{t+h}-X_{t}\right|>\varepsilon\right)=0$, para todo $t$ e $\epsilon>0$ (continuidade estocástica).

As seguintes propriedades são válidas [para provas ver Klöessner (2007)]:

(i) Se os retornos $\left(X_{t}\right)_{t \geq 0}$ são um processo de Lévy, então os vetores $\left(V_{\max }, V_{\text {min }}, r_{\text {dia }}\right)$ e $\left(V_{\text {min }}, V_{\text {max }}, r_{\text {dia }}\right)$ são identicamente distribuídos; e

(ii) Se os retornos $\left(X_{t}\right)_{t \geq 0}$ são um processo de Lévy, então temos que:

$$
\left(R_{\text {max }}, R_{\text {min }}\right) \stackrel{d}{=}\left(R_{\text {min }}, R_{\text {max }}\right) \text { e }\left(U_{\text {max }}, U_{\text {min }}\right) \stackrel{d}{=}\left(U_{\text {min }}, U_{\text {max }}\right)
$$

onde $\stackrel{d}{=}$ indica igualdade em distribuição.

Portanto, a caracterização do processo de retornos $\left(X_{t}\right)_{t \geq 0}$ como Lévy implica na permutabilidade dos vetores $\left(V_{\max }, V_{\min }\right),\left(R_{\max }, R_{\min }\right)$ e $\left(U_{\max }, U_{\min }\right)$, e o uso de testes de hipóteses que verifiquem a permutabilidade das versões amostrais dos mesmos é uma ferramenta para verificar se a hipótese de o processo ser de Lévy é condizente com os dados observados.

A aplicação de testes de permutabilidade a esses índices indicou que a hipótese pode ser rejeitada para uma grande parte das ações estudadas, indicando que os processos de Lévy não são plenamente adequados para descrever a dinâmica das variáveis analisadas. 


\subsection{Medida de Assimetria Bivariada Baseada nos Coeficientes de Correlação Condicional}

Nesta seção apresentamos uma motivação para mensurarmos o quão não-permutável é um vetor aleatório. Em seguida é proposta uma medida de não-permutabilidade baseada na diferença dos coeficientes de correlação condicionais dadas algumas partições $\left(\mathcal{F}^{-}, \mathcal{F}^{+}\right)$do espaço amostral, e exemplos de aplicação da mesma são analisados.

\subsubsection{Motivação e Definições}

Uma representação geral de qualquer vetor aleatório permutável (em termos da função geradora de momentos multivariada) é dada em Kolev e Paiva (2008). Os autores mostram que a permutabilidade de um vetor aleatório permutável $\left(X_{1}, \ldots, X_{n}\right)$ quadrado integrável tem uma estreita relação com a igualdade das correlações entre elementos consecutivos do vetor de variáveis, ou seja,

$$
\operatorname{Corr}\left(X_{i}, X_{i+1}\right)=\rho, \quad i=1,2, \ldots, n-1 .
$$

A hipótese de equicorrelação, em termos do processo aleatório, implica que o processo subjacente tenha incrementos independentes e estacionários (e.g. processos Poisson, Lévy, Markovianos). Assumir que o processo é Markoviano, por exemplo, implica na validade do modelo de independência condicional. Mas é de amplo conhecimento que tais modelos são muito restritivos para modelar a dinâmica de retornos financeiros, por exemplo.

Recentemente tem-se dado preferência na modelagem de incerteza, veja Kurowicka e Cooke (2006), a modelos de dependência condicional. Como o próprio nome indica, esses modelos não são condicionalmente dependentes, i.e. são não-permutáveis (assimétricos). Portanto, a mensuração de quão permutável é um vetor aleatório é um tópico de interesse.

O relacionamento estreito entre o conceito de permutabilidade e a correlação, como mostrado acima, servem de motivação para se explorar maneiras de identificar a permutabilidade por meio dessa medida de dependência. Em se tratando de assimetria bivariada, uma caracterização da permutabilidade em termos da equicorrelação não é útil, uma vez que há uma única correlação a ser estudada. Uma alternativa possível é utilizar coeficientes de correlação condicionais em porções reduzidas do espaço amostral e tentar estabelecer conexões entre o comportamento desses coeficientes e a permutabilidade do vetor aleatório bivariado estudado.

Tendo em vista esses fatos, a seguir é proposta uma medida de não-permutabilidade de um vetor aleatório bidimensional, baseada em coeficientes de correlação condicionais.

Definição 4.2. Seja $(X, Y)$ um vetor aleatório com distribuição conjunta $F(x, y)$ com segundos momentos finitos e seja $\left(\mathcal{F}^{-}, \mathcal{F}^{+}\right)$uma partição do espaço amostral tal que o conjunto de elementos em $\mathcal{F}^{-}$ou $\mathcal{F}^{+}$tem probabilidade estritamente positiva, isto é, seja $\mu_{F}$ a medida de induzida por $F(x, y)$, então $\mu_{F}\left(\left\{(x, y):(x, y) \in \mathcal{F}^{-}\right\}\right)>0$ e $\mu_{F}\left(\left\{(x, y):(x, y) \in \mathcal{F}^{+}\right\}\right)>0$. Dessa forma, a medida de não-permutabilidade proposta, $c$, é definida como:

$$
c: \stackrel{\operatorname{def}}{=} \frac{\left|\rho^{+}-\rho^{-}\right|}{2}
$$


onde

$$
\begin{aligned}
\rho^{+} & =\frac{E\left(X Y \mid \mathcal{F}^{+}\right)-E\left(X \mid \mathcal{F}^{+}\right) E\left(Y \mid \mathcal{F}^{+}\right)}{\sqrt{\left[E\left(X^{2} \mid \mathcal{F}^{+}\right)-E^{2}\left(X \mid \mathcal{F}^{+}\right)\right]\left[E\left(Y^{2} \mid \mathcal{F}^{+}\right)-E^{2}\left(Y \mid \mathcal{F}^{+}\right)\right]}}, \\
\rho^{-} & =\frac{E\left(X Y \mid \mathcal{F}^{-}\right)-E\left(X \mid \mathcal{F}^{-}\right) E\left(Y \mid \mathcal{F}^{-}\right)}{\sqrt{\left[E\left(X^{2} \mid \mathcal{F}^{-}\right)-E^{2}\left(X \mid \mathcal{F}^{-}\right)\right]\left[E\left(Y^{2} \mid \mathcal{F}^{-}\right)-E^{2}\left(Y \mid \mathcal{F}^{-}\right)\right]}}
\end{aligned}
$$

Algumas propriedades dessa medida, cujas demonstrações são triviais, são as seguintes:

(i) $\quad c \in[0,1] \mathrm{e}$

(ii) $\quad$ Se $F(x, y)=F(y, x)$ para todo ponto $(x, y) \in \bar{\Re}^{2}$ e $\mathcal{F}^{+}=\mathcal{F}^{+}(X-Y>0)$, então $c=0$. Neste caso $F(x, y)$ denota a distribuição conjunta de $(X, Y)$.

Seja $\left(\mathcal{F}^{-}, \mathcal{F}^{+}\right)$uma partição do espaço amostral $\Omega$, isto é, $\Omega=\mathcal{F}^{-} \cup \mathcal{F}^{+}$. Temos que

$$
\begin{gathered}
E(X Y)=\left(\rho^{+} \sigma_{X}^{+} \sigma_{Y}^{+}+\mu_{X}^{+} \mu_{Y}^{+}\right) P\left(\mathcal{F}^{+}\right)+\left(\rho^{-} \sigma_{X}^{-} \sigma_{Y}^{-}+\mu_{X}^{-} \mu_{Y}^{-}\right) P\left(\mathcal{F}^{-}\right) ; \\
\begin{array}{c}
\operatorname{Var}(X)=\left(\sigma_{X}^{+}\right)^{2} P\left(\mathcal{F}^{+}\right)+\left(\sigma_{X}^{-}\right)^{2} P\left(\mathcal{F}^{-}\right)+\left(\mu_{X}^{+}\right)^{2} P\left(\mathcal{F}^{+}\right)+\left(\mu_{X}^{-}\right)^{2} P\left(\mathcal{F}^{-}\right) \\
-\left[\mu_{X}^{+} P\left(\mathcal{F}^{+}\right)+\mu_{X}^{-} P\left(\mathcal{F}^{-}\right)\right]^{2} ;
\end{array}
\end{gathered}
$$

$\operatorname{Var}(Y)=\left(\sigma_{Y}^{+}\right)^{2} P\left(\mathcal{F}^{+}\right)+\left(\sigma_{Y}^{-}\right)^{2} P\left(\mathcal{F}^{-}\right)+\left(\mu_{Y}^{+}\right)^{2} P\left(\mathcal{F}^{+}\right)+\left(\mu_{Y}^{-}\right)^{2} P\left(\mathcal{F}^{-}\right)$

$$
-\left[\mu_{Y}^{+} P\left(\mathcal{F}^{+}\right)+\mu_{Y}^{-} P\left(\mathcal{F}^{-}\right)\right]^{2}
$$

$E(X)=\mu_{X}^{+} P\left(\mathcal{F}^{+}\right)+\mu_{X}^{-} P\left(\mathcal{F}^{-}\right)$e $E(Y)=\mu_{Y}^{+} P\left(\mathcal{F}^{+}\right)+\mu_{Y}^{-} P\left(\mathcal{F}^{-}\right)$,

onde $\mu_{A}^{s}=E\left(A \mid \mathcal{F}^{s}\right)$ e $\sigma_{A}^{s}=\sqrt{E\left(A^{2} \mid \mathcal{F}^{s}\right)-E^{2}\left(A \mid \mathcal{F}^{s}\right)}$, $\operatorname{com} A=X$ ou $Y, s= \pm, \rho^{+}, \rho^{-}$como definidos anteriormente e $P\left(\mathcal{F}^{s}\right)=P\left(\right.$ eventos em $\mathcal{F}^{s}$ ).

Teorema 4.4. Com as notações e relações mostradas acima, temos que o coeficiente de correlação de Pearson, $\rho=\operatorname{Corr}(X, Y)$, pode ser decomposto da seguinte maneira:

onde

$$
\rho=\rho_{0}+c^{-} \rho^{-}+c^{+} \rho^{+}
$$

$$
\begin{gathered}
\rho_{0}=\frac{\left(\mu_{X}^{+} \mu_{Y}^{+}+\mu_{X}^{-} \mu_{Y}^{-}-\mu_{X}^{+} \mu_{Y}^{-}-\mu_{X}^{-} \mu_{Y}^{+}\right) P\left(\mathcal{F}^{-}\right) P\left(\mathcal{F}^{+}\right)}{\sqrt{\operatorname{Var}(X) \operatorname{Var}(Y)}}, \\
c^{-}=\frac{\sigma_{X}^{-} \sigma_{Y}^{-} P\left(\mathcal{F}^{-}\right)}{\sqrt{\operatorname{Var}(X) \operatorname{Var}(Y)}} \text { e } c^{+}=\frac{\sigma_{X}^{+} \sigma_{Y}^{+} P\left(\mathcal{F}^{+}\right)}{\sqrt{\operatorname{Var}(X) \operatorname{Var}(Y)}} .
\end{gathered}
$$

Prova: É imediata, bastando realizar uma álgebra simples. 
Para exemplificar a aplicação da medida de assimetria definida acima e da decomposição do coeficiente de correlação, três casos serão analisados. O primeiro trata da distribuição normal bivariada $\mathcal{N}(\boldsymbol{\mu}, \boldsymbol{\Sigma})$ com condicionamento $\mathcal{F}^{+}=\mathcal{F}^{+}\left(X>\mu_{X}\right)$. Nos demais o condicionamento estudado é $\mathcal{F}^{+}=\mathcal{F}^{+}(X-Y>0)$ e as distribuições analisadas são a Normal bivariada $\mathcal{N}(\boldsymbol{\mu}, \boldsymbol{\Sigma})$ e a Normal Assimétrica bivariada $\mathcal{S} \mathcal{N}\left(\alpha^{\prime}, \beta^{\prime}, \omega^{\prime}\right)$.

No que segue os subscritos $N 1, N 2$ e $S$ presentes nos coeficientes $\rho^{+}$e $\rho^{+}$indicam, respectivamente, normalidade no caso 1 , normalidade no caso 2 e normalidade assimétrica. Adicionalmente, $\phi(x)$ e $\Phi(x)$ denotam, respectivamente, a densidade e a função distribuição da normal padrão e as esperanças $E\left(W, \mathcal{F}^{+}\right)$e $E\left(W, \mathcal{F}^{-}\right)$denotam $E\left(W \mid \mathcal{F}^{+}\right) P\left(\mathcal{F}^{+}\right)$e $E\left(W \mid \mathcal{F}^{-}\right) P\left(\mathcal{F}^{-}\right)$, respectivamente, para $W \in\left\{X, X^{2}, Y, Y^{2}, X Y\right\}$.

\subsubsection{Assimetria da distribuição Normal em torno da média $\mu_{X}$}

Nesta seção analisamos o comportamento da medida proposta, $c$, e da decomposição do coeficiente de correlação quando a distribuição é Normal bivariada e o condicionamento é dado por uma das variáveis ser maior que sua média. Para isso são calculadas as esperanças e variâncias condicionais, o que é feito na seguinte proposição.

Proposição 4.1. Seja $(X, Y)$ um vetor aleatório com distribuição $\mathcal{N}(\mu, \Sigma)$ e condicionamento $\mathcal{F}^{+}=\mathcal{F}^{+}\left(X>\mu_{X}\right)$.

Temos as seguintes esperanças e variâncias condicionais:

$$
\begin{array}{ll}
E\left(X \mid X>\mu_{X}\right)=\mu_{X}+\frac{2 \sigma_{X}}{\sqrt{2 \pi}} ; & E\left(X \mid X \leq \mu_{X}\right)=\mu_{X}-\frac{2 \sigma_{X}}{\sqrt{2 \pi}} ; \\
E\left(Y \mid X>\mu_{X}\right)=\mu_{Y}+\frac{2 \rho \sigma_{Y}}{\sqrt{2 \pi}} ; & E\left(Y \mid X \leq \mu_{X}\right)=\mu_{Y}-\frac{2 \rho \sigma_{Y}}{\sqrt{2 \pi}} ; \\
\operatorname{Var}\left(X \mid X>\mu_{X}\right)=\frac{(\pi-2)}{\pi} \sigma_{X}^{2} ; & \operatorname{Var}\left(X \mid X \leq \mu_{X}\right)=\frac{(\pi-2)}{\pi} \sigma_{X}^{2} ; \\
\operatorname{Var}\left(Y \mid X>\mu_{X}\right)=\frac{\left(\pi-2 \rho^{2}\right)}{\pi} \sigma_{Y}^{2} ; & \operatorname{Var}\left(Y \mid X \leq \mu_{X}\right)=\frac{\left(\pi-2 \rho^{2}\right)}{\pi} \sigma_{Y}^{2} ; \\
E\left(X Y \mid X>\mu_{X}\right)=\rho \sigma_{X} \sigma_{Y}+\mu_{X} \mu_{Y}+\frac{\left(2 \rho \mu_{X} \sigma_{Y}+2 \mu_{Y} \sigma_{X}\right)}{\sqrt{2 \pi}} ; \\
E\left(X Y \mid X \leq \mu_{X}\right)=\rho \sigma_{X} \sigma_{Y}+\mu_{X} \mu_{Y}-\frac{\left(2 \rho \mu_{X} \sigma_{Y}+2 \mu_{Y} \sigma_{X}\right)}{\sqrt{2 \pi}}
\end{array}
$$

Prova: Seguem os cálculos das esperanças e variâncias mencionadas.

1. Cálculo de $E\left(X \mid X>\mu_{X}\right)$ e $E\left(X \mid X \leq \mu_{X}\right)$ :

$$
\begin{aligned}
& E\left(X, X>\mu_{X}\right)=\int_{\mu_{X}}^{\infty} \frac{x e^{-\frac{1}{2}\left(\frac{x-\mu_{X}}{\sigma_{X}}\right)^{2}}}{\sigma_{X} \sqrt{2 \pi}} d x=\int_{0}^{\infty} \frac{\left(\sigma_{X} w+\mu_{X}\right) e^{-\frac{w^{2}}{2}}}{\sigma_{X} \sqrt{2 \pi}} d w=\frac{\mu_{X}}{2}+\frac{\sigma_{X}}{\sqrt{2 \pi}} . \\
& P\left(X>\mu_{X}\right)=\int_{\mu_{X}}^{\infty} \frac{e^{-\frac{1}{2}\left(\frac{x-\mu_{X}}{\sigma_{X}}\right)^{2}}}{\sigma_{X} \sqrt{2 \pi}} d x=\int_{0}^{\infty} \frac{e^{-\frac{w^{2}}{2}}}{\sqrt{2 \pi}} d w=\frac{1}{2} .
\end{aligned}
$$


Portanto $E\left(X \mid X>\mu_{X}\right)=\mu_{X}+\frac{2 \sigma_{X}}{\sqrt{2 \pi}}$.

Como $E(X)=E\left(X \mid X>\mu_{X}\right) P\left(X>\mu_{X}\right)+E\left(X \mid X \leq \mu_{X}\right) P\left(X \leq \mu_{X}\right)$, temos que

$$
E\left(X \mid X \leq \mu_{X}\right)=\mu_{X}-\frac{2 \sigma_{X}}{\sqrt{2 \pi}} .
$$

2. Cálculo de $E\left(Y \mid X>\mu_{X}\right)$ e $E\left(Y \mid X \leq \mu_{X}\right)$ :

$$
\begin{aligned}
E\left(Y, X>\mu_{X}\right) & =\int_{-\infty}^{\infty} \int_{\mu_{X}}^{\infty} y f_{X, Y}(x, y) d x d y=\int_{-\infty}^{\infty} y f_{Y}(y) \int_{\mu_{X}}^{\infty} f_{X \mid Y}(x \mid y) d x d y \\
& =\int_{-\infty}^{\infty} \frac{y e^{-\frac{1}{2}\left(\frac{y-\mu_{Y}}{\sigma_{Y}}\right)^{2}}}{\sigma_{Y} \sqrt{2 \pi}} \int_{\mu_{X}}^{\infty} \frac{e^{-\frac{1}{2}\left(\frac{x-\mu_{X}-\rho \frac{\sigma_{X}}{\sigma_{Y}}\left(y-\mu_{Y}\right)}{\sigma_{X} \sqrt{1-\rho^{2}}}\right)^{2}}}{\sigma_{X} \sqrt{2 \pi} \sqrt{1-\rho^{2}}} d x d y \\
& =\int_{-\infty}^{\infty} \frac{y e^{-\frac{1}{2}\left(\frac{y-\mu_{Y}}{\sigma_{Y}}\right)^{2}}}{\sigma_{Y} \sqrt{2 \pi}} \int_{\frac{-\rho\left(y-\mu_{Y}\right)}{\sigma_{Y} \sqrt{1-\rho^{2}}}}^{\frac{e^{-\frac{w^{2}}{2}}}{\sqrt{2 \pi}}} d w d y=\int_{-\infty}^{\infty} \frac{y e^{-\frac{1}{2}\left(\frac{y-\mu_{Y}}{\sigma_{Y}}\right)^{2}}}{\sigma_{Y} \sqrt{2 \pi}} \Phi\left(\frac{\rho\left(y-\mu_{Y}\right)}{\sigma_{Y} \sqrt{1-\rho^{2}}}\right) d y \\
& =\frac{\mu_{Y}}{2}+\frac{\rho \sigma_{Y}}{\sqrt{2 \pi}} .
\end{aligned}
$$

Portanto $E\left(Y \mid X>\mu_{X}\right)=\mu_{Y}+\frac{2 \rho \sigma_{Y}}{\sqrt{2 \pi}}$.

Como $E(Y)=E\left(Y \mid X>\mu_{X}\right) P\left(X>\mu_{X}\right)+E\left(Y \mid X \leq \mu_{X}\right) P\left(X \leq \mu_{X}\right)$, temos que

$$
E\left(Y \mid X \leq \mu_{X}\right)=\mu_{Y}-\frac{2 \rho \sigma_{Y}}{\sqrt{2 \pi}} .
$$

3. Cálculo de $E\left(X^{2} \mid X>\mu_{X}\right)$ e $E\left(X^{2} \mid X \leq \mu_{X}\right)$ :

$E\left(X^{2}, X>\mu_{X}\right)=\int_{\mu_{X}}^{\infty} \frac{x^{2} e^{-\frac{1}{2}\left(\frac{x-\mu_{X}}{\sigma_{X}}\right)^{2}}}{\sigma_{X} \sqrt{2 \pi}} d x=\int_{0}^{\infty} \frac{\left(\sigma_{X} w+\mu_{X}\right)^{2} e^{-\frac{w^{2}}{2}}}{\sigma_{X} \sqrt{2 \pi}} d w=\frac{\mu_{X}^{2}}{2}+\frac{2 \mu_{X} \sigma_{X}}{\sqrt{2 \pi}}+\frac{\sigma_{X}^{2}}{2}$.

Portanto $E\left(X^{2} \mid X>\mu_{X}\right)=\mu_{X}^{2}+\frac{4 \mu_{X} \sigma_{X}}{\sqrt{2 \pi}}+\sigma_{X}^{2}$.

Como $E\left(X^{2}\right)=E\left(X^{2} \mid X>\mu_{X}\right) P\left(X>\mu_{X}\right)+E\left(X^{2} \mid X \leq \mu_{X}\right) P\left(X \leq \mu_{X}\right)$ temos que

$$
E\left(X^{2} \mid X \leq \mu_{X}\right)=\sigma_{X}^{2}+\mu_{X}^{2}-\frac{4 \mu_{X} \sigma_{X}}{\sqrt{2 \pi}} .
$$


4. Cálculo de $E\left(Y^{2} \mid X>\mu_{X}\right)$ e $E\left(Y^{2} \mid X \leq \mu_{X}\right)$ :

$$
\begin{aligned}
E\left(Y^{2}, X>\mu_{X}\right) & \\
& =\int_{-\infty}^{\infty} \int_{\mu_{X}}^{\infty} y^{2} f_{X, Y}(x, y) d x d y=\int_{-\infty}^{\infty} y^{2} f_{Y}(y) \int_{\mu_{X}}^{\infty} f_{X \mid Y}(x \mid y) d x d y \\
& =\int_{-\infty}^{\infty} \frac{y^{2} e^{-\frac{1}{2}\left(\frac{y-\mu_{Y}}{\sigma_{Y}}\right)^{2}}}{\sigma_{Y} \sqrt{2 \pi}} \Phi\left(\frac{\rho\left(y-\mu_{Y}\right)}{\sigma_{Y} \sqrt{1-\rho^{2}}}\right) d y \\
& =\int_{-\infty}^{\infty} \frac{\left(\sigma_{Y} w+\mu_{Y}\right)^{2} e^{-\frac{w^{2}}{2}}}{\sqrt{2 \pi}} \Phi\left(\frac{\rho w}{\sqrt{1-\rho^{2}}}\right) d w=\frac{\mu_{Y}^{2}}{2}+\frac{2 \rho \mu_{Y} \sigma_{Y}}{\sqrt{2 \pi}}+\frac{\sigma_{Y}^{2}}{2} .
\end{aligned}
$$

Portanto $E\left(Y^{2} \mid X>\mu_{X}\right)=\mu_{Y}^{2}+\frac{4 \rho \mu_{Y} \sigma_{Y}}{\sqrt{2 \pi}}+\sigma_{Y}^{2}$.

Como $E\left(Y^{2}\right)=E\left(Y^{2} \mid X>\mu_{X}\right) P\left(X>\mu_{X}\right)+E\left(Y^{2} \mid X \leq \mu_{X}\right) P\left(X \leq \mu_{X}\right)$ temos que

$$
E\left(Y^{2} \mid X \leq \mu_{X}\right)=\sigma_{Y}^{2}+\mu_{Y}^{2}-\frac{4 \rho \mu_{Y} \sigma_{Y}}{\sqrt{2 \pi}} .
$$

5. Cálculo de $E\left(X Y \mid X>\mu_{X}\right)$ e $E\left(X Y \mid X \leq \mu_{X}\right)$ :

$$
\begin{aligned}
E\left(X Y, X>\mu_{X}\right) & =\int_{-\infty}^{\infty} \int_{\mu_{X}}^{\infty} x y f_{X, Y}(x, y) d x d y=\int_{-\infty}^{\infty} y f_{Y}(y) \int_{\mu_{X}}^{\infty} x f_{X \mid Y}(x \mid y) d x d y \\
= & \int_{-\infty}^{\infty} \frac{y e^{-\frac{1}{2}\left(\frac{y-\mu_{Y}}{\sigma_{Y}}\right)^{2}}}{\sigma_{Y} \sqrt{2 \pi}} \int_{\mu_{X}}^{\infty} \frac{x e^{-\frac{1}{2}\left(\frac{x-\mu_{X}-\rho \frac{\sigma_{X}}{\sigma_{Y}}\left(y-\mu_{Y}\right)}{\sigma_{X} \sqrt{1-\rho^{2}}}\right)^{2}}}{\sigma_{X} \sqrt{2 \pi} \sqrt{1-\rho^{2}}} d x d y \\
= & \int_{-\infty}^{\infty} \frac{y e^{-\frac{1}{2}\left(\frac{y-\mu_{Y}}{\sigma_{Y}}\right)^{2}}}{\sigma_{Y} \sqrt{2 \pi}} \int_{-\infty\left(y-\mu_{Y}\right)}^{\infty} \frac{\left(\sigma_{X} \sqrt{1-\rho^{2}} w+\mu_{X}+\rho \frac{\sigma_{X}}{\sigma_{Y}}\left(y-\mu_{Y}\right)\right) e^{-\frac{w^{2}}{2}}}{\sqrt{2 \pi}} d w d y \\
= & \int_{-\infty}^{\infty} \frac{y e^{-\frac{1}{2}\left(\frac{y-\mu_{Y}}{\sigma_{Y}}\right)^{2}}}{\sigma_{Y} \sqrt{2 \pi}}\left[\sigma_{X} \sqrt{1-\rho^{2}} \phi\left(\frac{\rho\left(y-\mu_{Y}\right)}{\sigma_{Y} \sqrt{1-\rho^{2}}}\right)\right. \\
+ & \left.\left(\mu_{X}+\rho \frac{\sigma_{X}}{\sigma_{Y}}\left(y-\mu_{Y}\right)\right) \Phi\left(\frac{\rho\left(y-\mu_{Y}\right)}{\sigma_{Y} \sqrt{1-\rho^{2}}}\right)\right] d y \\
= & \frac{\mu_{X} \mu_{Y}}{2}+\frac{\rho \sigma_{X} \sigma_{Y}}{2}+\frac{\rho \mu_{X} \sigma_{Y}}{\sqrt{2 \pi}}+\frac{\mu_{X} \sigma_{Y}}{\sqrt{2 \pi}} .
\end{aligned}
$$


Portanto $E\left(X Y \mid X>\mu_{X}\right)=\mu_{X} \mu_{Y}+\rho \sigma_{X} \sigma_{Y}+\frac{2\left(\rho \mu_{X} \sigma_{Y}+\mu_{Y} \sigma_{X}\right)}{\sqrt{2 \pi}}$.

Como $E(X Y)=E\left(X Y \mid X>\mu_{X}\right) P\left(X>\mu_{X}\right)+E\left(X Y \mid X \leq \mu_{X}\right) P\left(X \leq \mu_{X}\right)$ temos que

$$
E\left(X Y \mid X \leq \mu_{X}\right)=\mu_{X} \mu_{Y}+\rho \sigma_{X} \sigma_{Y}-\frac{2\left(\rho \mu_{X} \sigma_{Y}+\mu_{Y} \sigma_{X}\right)}{\sqrt{2 \pi}}
$$

Observação: Lien e Balakrishnan (2003) usam uma técnica similar para fazer uma análise de correlação condicional das estatísticas de ordem de uma distribuição Normal bivariada.

Corolário 4.1. A partir das expressões acima obtemos

e portanto temos que $c=0$.

$$
\rho_{N 1}^{+}=\rho_{N 1}^{-}=\rho \sqrt{\frac{\pi-2}{\pi-2 \rho^{2}}}<\rho,
$$

Os parâmetros da decomposição de $\rho=\rho_{0}+c^{-} \rho^{-}+c^{+} \rho^{+}$são:

$$
\rho_{0}=\frac{2 \rho}{\pi} \text { e } c^{-}=c^{+}=\frac{\sqrt{(\pi-2)\left(\pi-2 \rho^{2}\right)}}{2 \pi}
$$

Cabe notar que os resultados do corolário 4.1 são válidos para quaisquer parâmetros $\boldsymbol{\mu}$ e $\boldsymbol{\Sigma}$, apesar de claramente a distribuição normal não ser simétrica quando $\mu_{X} \neq \mu_{Y}$ ou $\sigma_{X} \neq \sigma_{Y}$.

Este exemplo mostra a importância do condicionamento analisado, uma vez que uma escolha sem relação direta com a forma de assimetria analisada pode levar a conclusões errôneas.

\subsubsection{Assimetria da distribuição Normal em torno da reta $y=x$.}

Nesta seção analisamos o comportamento da medida proposta, $c$, e da decomposição do coeficiente de correlação quando a distribuição é Normal bivariada e o condicionamento é dado por uma das variáveis ser maior que a outra. Para isso calculamos as esperanças e variâncias condicionais, o que é feito na seguinte proposição.

Proposição 4.2. Seja $(X, Y)$ um vetor aleatório com distribuição $\mathcal{N}(\boldsymbol{\mu}, \boldsymbol{\Sigma})$ e condicionamento $\mathcal{F}^{+}=\mathcal{F}^{+}(X-Y>0)$. Tomando $U=X-Y$, temos que:

$$
\begin{array}{lr}
E(U)=\mu_{X}-\mu_{Y}=\mu_{U} ; & \operatorname{Var}(U)=\sigma_{X}^{2}-2 \rho \sigma_{X} \sigma_{Y}+\sigma_{Y}^{2}=\sigma_{U}^{2} ; \\
\operatorname{Corr}(X, U)=\frac{\sigma_{X}-\rho \sigma_{Y}}{\sqrt{\sigma_{X}^{2}-2 \rho \sigma_{X} \sigma_{Y}+\sigma_{Y}^{2}}}=\rho_{U} ; & P(U>0)=\Phi\left(\frac{\mu_{U}}{\sigma_{U}}\right) .
\end{array}
$$


Temos as seguintes esperanças e variâncias condicionais:

$$
\begin{aligned}
& E(X \mid X-Y>0)=\mu_{X}+\frac{\rho_{U} \sigma_{X} \phi\left(\frac{\mu_{U}}{\sigma_{U}}\right)}{\Phi\left(\frac{\mu_{U}}{\sigma_{U}}\right)} \\
& E(X \mid X-Y \leq 0)=\mu_{X}-\frac{\rho_{U} \sigma_{X} \phi\left(\frac{\mu_{U}}{\sigma_{U}}\right)}{1-\Phi\left(\frac{\mu_{U}}{\sigma_{U}}\right)} \\
& E(Y \mid X-Y>0)=\mu_{X}-\mu_{U}+\frac{\left(\rho_{U} \sigma_{X}-\sigma_{U}\right) \phi\left(\frac{\mu_{U}}{\sigma_{U}}\right)}{\Phi\left(\frac{\mu_{U}}{\sigma_{U}}\right)} \\
& E(Y \mid X-Y \leq 0)=\mu_{X}-\mu_{U}-\frac{\left(\rho_{U} \sigma_{X}-\sigma_{U}\right) \phi\left(\frac{\mu_{U}}{\sigma_{U}}\right)}{1-\Phi\left(\frac{\mu_{U}}{\sigma_{U}}\right)} ; \\
& \operatorname{Var}(X \mid X-Y>0)=\sigma_{X}^{2}-\frac{\rho_{U}^{2} \sigma_{X}^{2} \phi\left(\frac{\mu_{U}}{\sigma_{U}}\right)}{\sigma_{U} \Phi\left(\frac{\mu_{U}}{\sigma_{U}}\right)}\left[\mu_{U}+\frac{\sigma_{U} \phi\left(\frac{\mu_{U}}{\sigma_{U}}\right)}{\Phi\left(\frac{\mu_{U}}{\sigma_{U}}\right)}\right] \\
& \operatorname{Var}(X \mid X-Y \leq 0)=\sigma_{X}^{2}+\frac{\rho_{U}^{2} \sigma_{X}^{2} \phi\left(\frac{\mu_{U}}{\sigma_{U}}\right)}{\sigma_{U}\left(1-\Phi\left(\frac{\mu_{U}}{\sigma_{U}}\right)\right)}\left[\mu_{U}-\frac{\sigma_{U} \phi\left(\frac{\mu_{U}}{\sigma_{U}}\right)}{1-\Phi\left(\frac{\mu_{U}}{\sigma_{U}}\right)}\right] \\
& \operatorname{Var}(Y \mid X-Y>0)=\sigma_{X}^{2}-2 \rho_{U} \sigma_{X} \sigma_{U}+\sigma_{U}^{2}-\frac{\left(\rho_{U} \sigma_{X}-\sigma_{U}\right)^{2} \phi\left(\frac{\mu_{U}}{\sigma_{U}}\right)}{\sigma_{U} \Phi\left(\frac{\mu_{U}}{\sigma_{U}}\right)}\left[\mu_{U}+\frac{\sigma_{U} \phi\left(\frac{\mu_{U}}{\sigma_{U}}\right)}{\Phi\left(\frac{\mu_{U}}{\sigma_{U}}\right)}\right] ; \\
& \operatorname{Var}(Y \mid X-Y \leq 0)=\sigma_{X}^{2}-2 \rho_{U} \sigma_{X} \sigma_{U}+\sigma_{U}^{2}+\frac{\left(\rho_{U} \sigma_{X}-\sigma_{U}\right)^{2} \phi\left(\frac{\mu_{U}}{\sigma_{U}}\right)}{\sigma_{U}\left(1-\Phi\left(\frac{\mu_{U}}{\sigma_{U}}\right)\right)}\left[\mu_{U}-\frac{\sigma_{U} \phi\left(\frac{\mu_{U}}{\sigma_{U}}\right)}{1-\Phi\left(\frac{\mu_{U}}{\sigma_{U}}\right)}\right] \\
& E(X Y \mid X-Y>0)=\sigma_{X}^{2}+\mu_{X}^{2}-\mu_{X} \mu_{U}-\rho_{U} \sigma_{X} \sigma_{U}+\frac{\left[2 \rho_{U} \sigma_{X} \mu_{X}-\rho_{U}^{2} \sigma_{X}^{2}\left(\frac{\mu_{U}}{\sigma_{U}}\right)-\mu_{X} \sigma_{U}\right] \phi\left(\frac{\mu_{U}}{\sigma_{U}}\right)}{\Phi\left(\frac{\mu_{U}}{\sigma_{U}}\right)} ; \\
& E(X Y \mid X-Y \leq 0)=\sigma_{X}^{2}+\mu_{X}^{2}-\mu_{X} \mu_{U}-\rho_{U} \sigma_{X} \sigma_{U}-\frac{\left[2 \rho_{U} \sigma_{X} \mu_{X}-\rho_{U}^{2} \sigma_{X}^{2}\left(\frac{\mu_{U}}{\sigma_{U}}\right)-\mu_{X} \sigma_{U}\right] \phi\left(\frac{\mu_{U}}{\sigma_{U}}\right)}{1-\Phi\left(\frac{\mu_{U}}{\sigma_{U}}\right)} .
\end{aligned}
$$


Prova: Para uma demonstração dos cálculos acima ver Apêndice A1.

A partir das expressões acima observamos que quando ocorre igualdade das médias $\left(\mu_{X}=\mu_{Y}\right)$ as correlações condicionais $\rho^{+}, \rho^{-}$são idênticas e, portanto, temos que $c=0$. Duas situações distintas merecem atenção: $\mu_{X}=\mu_{Y}, \sigma_{X} \neq \sigma_{Y}$ e $\mu_{X}=\mu_{Y}, \sigma_{X}=\sigma_{Y}$, dadas no seguinte corolário.

Corolário 4.2. Seja $\mu_{X}=\mu_{Y}, \sigma_{X} \neq \sigma_{Y}$, temos que

$$
\rho_{N 2}^{+}=\rho_{N 2}^{-}=\frac{\rho \pi\left(\sigma_{X}^{2}-2 \rho \sigma_{X} \sigma_{Y}+\sigma_{Y}^{2}\right)+2\left(\sigma_{X}-\rho \sigma_{Y}\right)\left(\sigma_{Y}-\rho \sigma_{X}\right)}{\sqrt{\left[\pi\left(\sigma_{X}^{2}-2 \rho \sigma_{X} \sigma_{Y}+\sigma_{Y}^{2}\right)-2\left(\sigma_{X}-\rho \sigma_{Y}\right)^{2}\right]\left[\pi\left(\sigma_{X}^{2}-2 \rho \sigma_{X} \sigma_{Y}+\sigma_{Y}^{2}\right)-2\left(\sigma_{Y}-\rho \sigma_{X}\right)^{2}\right]}} .
$$

Adicionalmente, os parâmetros da decomposição de $\rho$ são:

$$
\begin{gathered}
\rho_{0}=\frac{2\left(\sigma_{X}-\rho \sigma_{Y}\right)\left(\sigma_{Y}-\rho \sigma_{X}\right)}{\pi\left(\sigma_{X}^{2}-2 \rho \sigma_{X} \sigma_{Y}+\sigma_{Y}^{2}\right)} \mathrm{e} \\
c^{-}=c^{+}=\frac{\sqrt{\left[\pi\left(\sigma_{X}^{2}-2 \rho \sigma_{X} \sigma_{Y}+\sigma_{Y}^{2}\right)-2\left(\sigma_{X}-\rho \sigma_{Y}\right)^{2}\right]\left[\pi\left(\sigma_{X}^{2}-2 \rho \sigma_{X} \sigma_{Y}+\sigma_{Y}^{2}\right)-2\left(\sigma_{Y}-\rho \sigma_{X}\right)^{2}\right]}}{2 \pi\left(\sigma_{X}^{2}-2 \rho \sigma_{X} \sigma_{Y}+\sigma_{Y}^{2}\right)} .
\end{gathered}
$$

Seja $\mu_{X}=\mu_{Y}, \sigma_{X}=\sigma_{Y}$, temos que

$$
\rho_{N 2}^{+}=\rho_{N 2}^{-}=\frac{1+\rho(\pi-1)}{\pi-1+\rho}>\rho .
$$

Adicionalmente temos que os parâmetros da decomposição de $\rho$ são:

$$
\rho_{0}=\frac{1-\rho}{\pi} \text { e } c^{-}=c^{+}=\frac{\pi-(1-\rho)}{2 \pi} .
$$

Portanto, apesar de a distribuição normal não ser simétrica quando $\mu_{X}=\mu_{Y}, \sigma_{X} \neq \sigma_{Y}$, a medida de assimetria não demonstra esse fato, uma vez que $c=0$.

\subsubsection{Assimetria da distribuição Normal Assimétrica em torno da reta $y=x$}

Nesta seção analisamos o comportamento da medida proposta, $c$, e da decomposição do coeficiente de correlação quando a distribuição é Normal assimétrica bivariada e o condicionamento é dado por uma das variáveis ser maior que a outra, no caso especial onde as variáveis têm a mesma média. Para isso calculamos as esperanças e variâncias condicionais, o que é feito na seguinte proposição. 
Proposição 4.3. Seja $(X, Y)$ um vetor com distribuição $\mathcal{S} \mathcal{N}\left(\alpha^{\prime}, \beta^{\prime}, \omega^{\prime}\right)$ e condicionamento $\mathcal{F}^{+}=\mathcal{F}^{+}(X-Y>0)$, onde $\mathcal{S N}\left(\alpha^{\prime}, \beta^{\prime}, \omega^{\prime}\right)$ denota a distribuição normal assimétrica de parâmetros $\left(\alpha^{\prime}, \beta^{\prime}, \omega^{\prime}\right)$ cuja densidade é expressa por:

$$
f_{\mathcal{S N}}(x, y)=2 \phi_{\omega^{\prime}}(x, y) \Phi\left(\alpha^{\prime} x+\beta^{\prime} y\right), \quad(x, y) \in \Re^{2}
$$

Então se $E(X)=E(Y)$, temos que

$$
\rho_{S}^{+}=\rho_{S}^{-}=\rho_{N 2}^{+}=\rho_{N 2}^{-}=\frac{1+\rho(\pi-1)}{\pi-1+\rho}>\rho
$$

onde $\rho_{N 2}^{+}, \rho_{N 2}^{-}$representam os coeficientes de correlação condicionais com $\mathcal{F}^{+}=\mathcal{F}^{+}(X-Y>0)$ para o caso Normal bivariado de mesmas médias e variâncias.

Da relação acima segue também que $c=0$ e que a decomposição de $\rho$ em função de $\rho_{S}^{+}$e $\rho_{S}^{-}$tem os seguintes coeficientes

$$
\rho_{0}=\frac{1-\rho}{\pi} \text { e } c^{-}=c^{+}=\frac{\pi-(1-\rho)}{2 \pi} .
$$

Prova: A partir das propriedades básicas de $\mathcal{S N}\left(\alpha^{\prime}, \beta^{\prime}, \omega^{\prime}\right)$ encontradas no apêndice $\mathrm{A} 2$, temos que $E(X)=E(Y) \Leftrightarrow \alpha^{\prime}=\beta^{\prime}$. Como $\operatorname{Var}(X)=1-E^{2}(X)$ e $\operatorname{Var}(Y)=1-E^{2}(Y)$, temos que

$$
E(X)=E(Y) \Leftrightarrow \operatorname{Var}(X)=\operatorname{Var}(Y)
$$

A partir dos cálculos dos componentes dos coeficientes condicionais de correlação localizados neste mesmo apêndice, temos que para

$$
\alpha=\alpha^{\prime}+\beta^{\prime}, \quad \beta=-\beta^{\prime} \sqrt{1-2 \omega^{\prime}} \quad \text { e } \quad \omega=\sqrt{\frac{1-\omega^{\prime}}{2}},
$$

temos as seguintes quantidades:

$$
\begin{aligned}
& P(X-Y>0)=\frac{1}{2} \\
& E(X, X-Y>0)=E(Y, X-Y \leq 0)=\frac{\alpha\left(1-\omega^{2}\right)}{\sqrt{2 \pi} \sqrt{1+\alpha^{2}\left(1-\omega^{2}\right)}}+\frac{\omega}{\sqrt{2 \pi}} \\
& E(X, X-Y \leq 0)=E(Y, X-Y>0)=\frac{\alpha\left(1-\omega^{2}\right)}{\sqrt{2 \pi} \sqrt{1+\alpha^{2}\left(1-\omega^{2}\right)}}-\frac{\omega}{\sqrt{2 \pi}} \\
& E\left(X^{2}, X-Y>0\right)=E\left(Y^{2}, X-Y \leq 0\right)=\frac{1}{2}+\frac{2 \alpha \omega\left(1-\omega^{2}\right)}{\pi \sqrt{1+\alpha^{2}\left(1-\omega^{2}\right)}} \\
& E\left(X^{2}, X-Y \leq 0\right)=E\left(Y^{2}, X-Y>0\right)=\frac{1}{2}-\frac{2 \alpha \omega\left(1-\omega^{2}\right)}{\pi \sqrt{1+\alpha^{2}\left(1-\omega^{2}\right)}} \\
& E(X Y, X-Y>0)=E(X Y, X-Y \leq 0)=\frac{1-2 \omega^{2}}{2} .
\end{aligned}
$$


Portanto

$$
\begin{aligned}
\rho_{S}^{+}=\rho_{S}^{-} & =\frac{\left[1+\alpha^{2}\left(1-\omega^{2}\right)\right]\left[2 \omega^{2}(1-\pi)+\pi\right]-2 \alpha^{2}\left(1-\omega^{2}\right)^{2}}{\left[1+\alpha^{2}\left(1-\omega^{2}\right)\right]\left(\pi-2 \omega^{2}\right)-2 \alpha^{2}\left(1-\omega^{2}\right)^{2}} \\
& =\frac{\left[1+\omega^{\prime}(\pi-1)\right]+2 \alpha^{\prime 2} \omega^{\prime}\left(1+\omega^{\prime}\right)(\pi-2)}{\left[\pi-\left(1-\omega^{\prime}\right)\right]+2 \alpha^{\prime 2}\left(1+\omega^{\prime}\right)(\pi-2)} .
\end{aligned}
$$

Mas tem-se que

$$
\rho=\frac{\pi \omega^{\prime}\left[1+2 \alpha^{\prime 2}\left(1+\omega^{\prime}\right)\right]-2 \alpha^{\prime 2}\left(1+\omega^{\prime}\right)^{2}}{\pi\left[1+2 \alpha^{\prime 2}\left(1+\omega^{\prime}\right)\right]-2 \alpha^{\prime 2}\left(1+\omega^{\prime}\right)^{2}} .
$$

Portanto temos que

$$
\rho_{S}^{+}=\rho_{S}^{-}=\frac{1+\rho(\pi-1)}{\pi-1+\rho}>\rho .
$$

Quando $E(X)=E(Y)$ e $\operatorname{Var}(X)=\operatorname{Var}(Y)$, temos que

$$
\rho_{N 2}^{+}=\rho_{N 2}^{-}=\frac{1+\rho(\pi-1)}{\pi-1+\rho}, \quad \rho_{0}=\frac{1-\rho}{\pi} \text { e } c^{-}=c^{+}=\frac{\pi-(1-\rho)}{2 \pi} .
$$

Portanto o resultado segue.

Temos, portanto, que quando as variâncias e médias de $X$ e $Y$ são idênticas, a assimetria presente nas marginais não impacta nos coeficientes de correlação condicionais $\rho^{+}$e $\rho^{-}$, sendo o caso Normal bivariado assimétrico idêntico ao simétrico.

Assim como um valor zero de coeficiente de correlação (e também dos coeficientes Kendall $\tau$ e Spearman $\rho$ ) não implica na independência, os exemplos acima mostram que o mesmo ocorre com a medida $c$ em relação à permutabilidade.

Uma possível explicação para este fato são as fragilidades do coeficiente de correlação de Pearson, que devem influir na medida de assimetria, apesar de ser uma boa medida de dependência para as distribuições estudadas.

A medida de dependência linear de Bairamov e Kotz, e sua interpretação como um coeficiente de correlação condicional pontual, sugere uma outra explicação: o caráter demasiadamente global da medida $c=\frac{\left|\rho^{+}-\rho^{-}\right|}{2}$. Aplicando a medida de Bairamov e Kotz $(B K(x, y))$ à distribuição Normal Bivariada $\mathcal{N}\left(\mu_{X}, \mu_{Y}, \sigma_{X}, \sigma_{Y}, \rho_{X, Y}\right)$, conforme exemplo do capítulo 2 , temos

$$
B K(x, y)=\frac{\sigma_{X} \sigma_{Y} \rho_{X, Y}+\rho_{X, Y}^{2}\left(x-\mu_{X}\right)\left(y-\mu_{Y}\right)}{\sqrt{\left[\sigma_{X}^{2}+\rho_{X, Y}^{2}\left(x-\mu_{X}\right)^{2}\right]} \sqrt{\left[\sigma_{Y}^{2}+\rho_{X, Y}^{2}\left(y-\mu_{Y}\right)^{2}\right]}},
$$

e claramente $B K(x, y) \neq B K(y, x)$ quando $\mu_{X} \neq \mu_{Y}$ ou $\sigma_{X} \neq \sigma_{Y}$. 
Dessa forma, o problema que se põe é o de criar medidas que não apresentem essa deficiência, sendo iguais a um valor pré-determinado, usualmente zero, se e somente se o vetor é permutável.

A contribuição original desta seção foi propor uma medida de assimetria bastante flexível, sendo possível analisar diferentes formas de assimetria através de uma mudança no condicionamento. Além disso, foram obtidas propriedades da distribuição normal e normal assimétricas até então desconhecidas, e em alguns casos contra-intuitivas.

\subsection{O Conceito de Quantil Bivariado aplicado à Identificação de Não-Permutabilidade e Assimetria Radial}

O conceito de curvas quantis bivariadas é explorado nesta seção como forma de mensurar e testar a permutabilidade e a simetria radial da estrutura de dependência de dados bivariados contínuos. Primeiramente será definido o conceito de simetria radial e na seqüência será tratado o conceito de Transformada Integral de Probabilidade bivariada. A seguir as curvas quantis são definidas, suas propriedades são estudadas e a sua relação com a Transformada Integral de Probabilidade bivariada é estabelecida. A partir deste arcabouço teórico, são definidas medidas de não-permutabilidade e assimetria radial da cópula subjacente aos dados, bem como estabelecidos testes de hipóteses não-paramétricos para identificar a presença dessas modalidades de assimetria.

\subsubsection{Simetria Radial}

Sejam $X$ uma variável aleatória e $a$ um número real, então $X$ é dita simétrica em torno de $a$ se as variáveis aleatórias $X-a$ e $a-X$ têm a mesma distribuição. Analogamente, um vetor aleatório bivariado $(X, Y)$ é dito radialmente simétrico ao redor do ponto $(a, b) \in \mathfrak{R}^{2}$ se a distribuição conjunta de $X-a$ e $Y-b$ é a mesma distribuição de $a-X$ e $b-Y$.

O seguinte teorema caracteriza a simetria em termos da cópula subjacente à distribuição de $(X, Y)$.

Teorema 4.5. [Nelsen (2006)] Seja $(X, Y)$ um vetor aleatório contínuo com função distribuição conjunta $F$, marginais $F_{X}$ e $F_{Y}$ e cópula $C$. Adicionalmente, suponha que $X$ e $Y$ sejam simétricas em torno de $a$ e $b$, respectivamente. Então $(X, Y)$ é radialmente simétrico ao redor do ponto $(a, b) \in \mathfrak{R}^{2}$ se e somente $C$ satisfaz a seguinte equação funcional:

$$
C(u, v)=u+v-1+C(1-u, 1-v), \text { para todo }(u, v) \text { em }[0,1]^{2}
$$


A suposição de simetria radial desempenha um papel importante em aplicações em finanças, onde muitas vezes se assume que as "caudas bivariadas" tenham comportamento idêntico, permitindo tratar portfólios bivariados com posições compradas da mesma maneira que os com posições vendidas.

\subsubsection{Transformada Integral de Probabilidade Bivariada}

Nelsen et al. (2001) estudou as funções distribuição de cópulas, que tem como caso especial a Transformada Integral de Probabilidade bivariada. Os principais resultados que envolvem esse conceito são examinados e servem de base para desenvolvimentos deste capítulo.

Convencionaremos ser $\mu_{H}$ a medida em $\mathfrak{R}^{2}$ induzida pela função distribuição conjunta bivariada $H$, ou em termos de cópula, $\mu_{C}$ é a medida em $[0,1]^{2}$ induzida pela cópula $C$.

Definição 4.3. Sejam $H_{a}$ e $H_{b}$ funções distribuição bivariadas com marginais contínuas comuns $F$ e $G$. Sejam $X$ e $Y$ variáveis aleatórias cuja função distribuição conjunta é $H_{b}$ e seja $\left\langle H_{a} \mid H_{b}\right\rangle(X, Y)$ a variável aleatória $H_{a}(X, Y)$. A $H_{b}$-função distribuição de $H_{a}$, que será denotada por $\left(H_{a} \mid H_{b}\right)$, é dada por:

$$
\left(H_{a} \mid H_{b}\right)(t)=P\left(\left\langle H_{a} \mid H_{b}\right\rangle(X, Y) \leq t\right)=\mu_{H_{b}}\left(\left\{(x, y) \in \mathfrak{R}^{2} \mid H_{a}(x, y) \leq t\right\}\right), t \in[0,1] .
$$

No caso especial onde $H_{a}=H_{b}$, temos que $\left(H_{a} \mid H_{b}\right)(t)$ é o análogo bivariado da Transformada Integral de Probabilidade.

Uma definição análoga para cópulas é possível já que as cópulas são funções distribuição conjuntas. Dessa maneira, se $C_{a}$ e $C_{b}$ forem cópulas quaisquer e $U$ e $V$ forem variáveis aleatórias uniformes em $(0,1)$ com função distribuição conjunta $C_{b}$, então $\left\langle C_{a} \mid C_{b}\right\rangle(U, V)$ denota a variável $C_{a}(U, V)$ e a $C_{a}$-função distribuição de $C_{a}$ é dada por:

$$
\left(C_{a} \mid C_{b}\right)(t)=P\left(\left\langle C_{a} \mid C_{b}\right\rangle(U, V) \leq t\right)=\mu_{C_{b}}\left(\left\{(u, v) \in[0,1]^{2} \mid C_{a}(u, v) \leq t\right\}\right), t \in[0,1]
$$

O teorema a seguir faz a ligação entre a definição 4.3 acima aplicada às funções distribuição conjuntas e às cópulas.

Teorema 4.6. [Nelsen et al. (2001)] Sejam $H_{a}, H_{b}, F, G, X$ e $Y$ como na definição acima e sejam $C_{a}$ e $C_{b}$ as cópulas subjacentes a $H_{a}$ e $H_{b}$ respectivamente. Então $\left(H_{a} \mid H_{b}\right)(t)=\left(C_{a} \mid C_{b}\right)(t)$.

Este teorema mostra que a Transformada Integral de Probabilidade bivariada pode ser analisada via a cópula subjacente à função distribuição de $X$ e $Y$, sem a necessidade de levar em conta as distribuições marginais. 
Exemplo 4.2. [Nelsen et al. (2001)] Sejam $M, \Pi$ e $W$ as cópulas de comonotonicidade, independência e contracomonotonicidade, respectivamente. Calculando-se $\left(C_{a} \mid C_{b}\right)(t)$ para $C_{a}, C_{b} \in\{M, \Pi, W\}$ temos na tabela 4.2 um resumo dos resultados.

Tabela 4.2. $\quad\left(C_{a} \mid C_{b}\right)(t)$

\begin{tabular}{|c|c|c|c|}
\hline$\left(C_{a} \mid C_{b}\right)$ & $M$ & $\Pi$ & $W$ \\
\hline$M$ & $t$ & $2 t-t^{2}$ & $\operatorname{Min}(2 t, 1)$ \\
\hline$\Pi$ & $\sqrt{t}$ & $t[1-\ln (t)]$ & $1-\sqrt{\operatorname{Max}(0,1-4 t)}$ \\
\hline$W$ & $\frac{(t+1)}{2}$ & $1-\frac{(1-t)^{2}}{2}$ & 1 \\
\hline
\end{tabular}

Pode ser visto que a Transformada Integral de Probabilidade bivariada produz a distribuição uniforme em $(0,1)$ somente quando $C_{a}=C_{b}=M$, quando reproduz o caso univariado.

Sejam $C_{a}(u, v)$ uma cópula bivariada qualquer, $C_{b}(u, v)$ e $C_{b, i}(u, v)$ cópulas bivariadas tais que:

$$
C_{b}(u, v)=\sum_{i=1}^{n} \alpha_{i} C_{b, i}(u, v),
$$

$\operatorname{com} 0 \leq \alpha_{i} \leq 1, i=1, \ldots, n$ e $\sum_{i=1}^{n} \alpha_{i}=1$

Temos, pela linearidade de $\left(C_{a} \mid C_{b}\right)(t)$ em relação à medida $C_{b}$, que:

$$
\left(C_{a} \mid C_{b}\right)(t)=\sum_{i=1}^{n} \alpha_{i}\left(C_{a} \mid C_{b, i}\right)(t) .
$$

A ligação entre as funções distribuição de cópulas e as medidas de dependência Kendall $\tau$ e Spearman $\rho$ é feita no seguinte teorema.

Teorema 4.7. [Nelsen et al. (2001)] Sejam $X$ e $Y$ variáveis aleatórias contínuas com cópula $C$ e sejam $\tau_{C}$ e $\rho_{C}$ as versões populacionais das medidas de dependência Kendall $\tau$ e Spearman $\rho$ respectivamente. Então

$$
\begin{aligned}
& \tau_{C}=Q(C, C)=3-4 \int_{0}^{1}(C \mid C)(t) d t ; \\
& \rho_{C}=3 Q(C, \Pi)=9-12 \int_{0}^{1}(C \mid \Pi)(t) d t .
\end{aligned}
$$


Os próximos teoremas apresentam limites funcionais máximos e mínimos para $(C \mid C)(t)$ e caracterizam quais cópulas $C$ atingem esses limites.

Teorema 4.8. [Capéraà et al. (1997)] Seja $C$ uma cópula bivariada. Então a seguinte desigualdade é válida:

$$
t \leq(C \mid C)(t) \leq 1
$$

Teorema 4.9. [Nelsen et al. (2003)] Sejam $C$ uma cópula bivariada e $M$ e $W$ as cópulas comonotônicas e contramonotônicas, respectivamente. Se $(C \mid C)(t)=t$ para todo $t \in[0,1]$, então $C=M$, e se $(C \mid C)(t)=1$ para todo $t \in[0,1]$, então $C=W$.

\subsubsection{Definições e Conceitos das Curvas Quantis}

No caso univariado tem-se a definição usual do quantil de ordem $u$ da variável $X$, denotado por $Q_{X}(u)$, como

$$
Q_{X}(u)=F_{X}^{-1}(u)=\operatorname{Inf}\left\{x: F_{X}(x) \geq u\right\}
$$

Nesta subseção serão apresentadas, seguindo Belzunce et al. (2007), uma versão bivariada para o conceito de quantil e sua relação com o conceito de cópulas.

Definição 4.4. Sejam $\boldsymbol{X}=(X, Y)$ um vetor aleatório e $(x, y)$ um ponto em $\mathfrak{R}^{2}$. Denotemos por $F_{\varepsilon}(x, y), \varepsilon \in\{--,-+,+-,++\}$, as seguintes probabilidades:

$$
\begin{array}{ll}
F_{--}(x, y)=P(X \leq x, Y \leq y) ; & F_{++}(x, y)=P(X>x, Y>y) \\
F_{-+}(x, y)=P(X \leq x, Y>y) ; & F_{+-}(x, y)=P(X>x, Y \leq y) .
\end{array}
$$

Definição 4.5. Sejam $\boldsymbol{X}=(X, Y)$ um vetor aleatório contínuo e $p \in[0,1]$. Definimos o $p$-ésimo conjunto quantil bivariado, ou curva quantil, para a direção $\varepsilon$ como

$$
Q_{\mathbf{X}}(p, \varepsilon)=\left\{(x, y) \in \mathfrak{R}^{2}: F_{\varepsilon}(x, y)=p\right\}
$$

Exemplo 4.3. [Belzunce et al. (2007)] Seja $\boldsymbol{X}=(X, Y)$ um vetor aleatório com componentes independentes e identicamente distribuídos conforme exponenciais de parâmetro $\lambda=1$. Para um dado $p$, o cálculo das curvas quantis é feito pela solução da equação $F_{\varepsilon}(x, y)=p$ para 
todos $\varepsilon \in\{--,-+,+-,++\}$. As curvas para este exemplo são mostradas na figura abaixo:

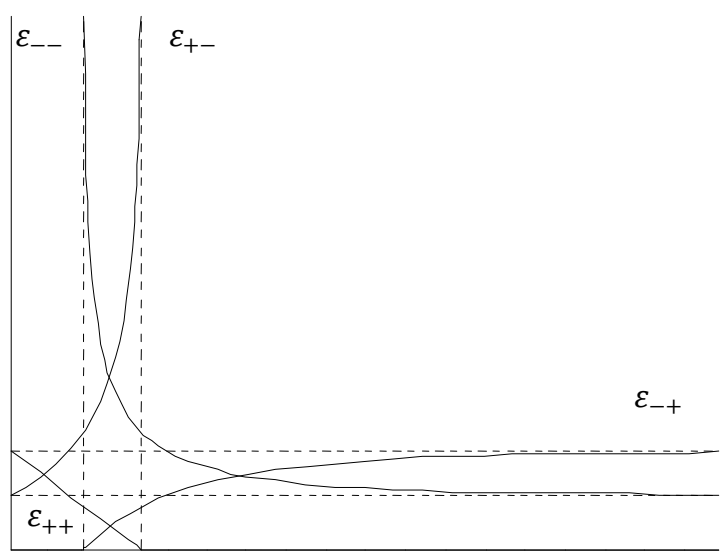

(a) $p \leq \frac{1}{2}$.

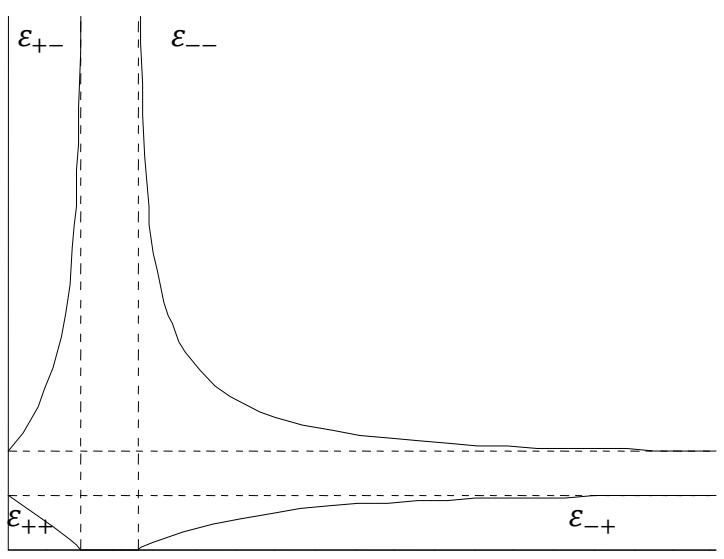

(b) $\boldsymbol{p}>\frac{1}{2}$.

Figura 4.1: Curvas quantis bivariadas: (a) $p \leq \frac{1}{2}$, (b) $p>\frac{1}{2}$.

Definição 4.6. Seja $X=(X, Y)$ um vetor aleatório contínuo e $p \in\left(\frac{1}{2}, 1\right]$. A região central de ordem $p$, denotada por $\Omega_{X}(p)$, é definida como

$$
\Omega_{X}(p)=\left\{(x, y) \in \mathfrak{R}^{2}: F_{\varepsilon}(x, y)<p, \quad \forall \varepsilon \in\{--,-+,+-,++\}\right\} .
$$

Observação: A região $\Omega_{X}(p)$ corresponde aos pontos no plano que acumulam probabilidade inferior a $p$ em todos os quadrantes.

Definição 4.7. Sejam $\boldsymbol{X}=(X, Y)$ um vetor aleatório contínuo e $p \in[0,1]$. Definimos a região lateral de ordem $p$ na direção $\varepsilon$, denotada por $L_{X}(p, \varepsilon)$, como

$$
L_{X}(p, \varepsilon)=\left\{(x, y) \in \Re^{2}: F_{\varepsilon}(x, y)>p\right\}
$$

Lema 4.1. [Belzunce et al. (2007)] Seja $\boldsymbol{X}=(X, Y)$ um vetor aleatório contínuo com cópula $C$ e sejam $u, v \in(0,1)$. Então a probabilidade $F_{\varepsilon}\left(Q_{X}(u), Q_{Y}(v)\right)$ depende somente da cópula $C$ para cada direção $\varepsilon$.

Prova: Usando as relações usuais da teoria de cópulas temos que 


$$
F_{\varepsilon}\left(Q_{X}(u), Q_{Y}(v)\right)= \begin{cases}C(u, v), & \varepsilon=\varepsilon_{--}, \\ u-C(u, v), & \varepsilon=\varepsilon_{-+}, \\ v-C(u, v), & \varepsilon=\varepsilon_{+-} \\ 1-u-v+C(u, v), & \varepsilon=\varepsilon_{++}\end{cases}
$$

Teorema 4.10. [Belzunce et al. (2007)] Seja $\boldsymbol{X}=(X, Y)$ um vetor aleatório contínuo com cópula $C$. Então a probabilidade acumulada na região central de ordem $p, P\left(\boldsymbol{X} \in \Omega_{\boldsymbol{X}}(p)\right)$, depende somente de $C$.

Prova: Temos que

$$
\begin{aligned}
P\left(X \in \Omega_{X}(p)\right) & =\mu_{F}\left(\left\{(x, y) \in \mathfrak{R}^{2}: F_{\varepsilon}(x, y)<p, \text { para } \varepsilon \in\{--,-+,+-,++\}\right\}\right), \\
& =\mu_{C}\left(\left\{(u, v) \in[0,1]^{2}: F_{\varepsilon}\left(Q_{X}(u), Q_{Y}(v)\right)<p \text {, para } \varepsilon \in\{--,-+,+-,++\}\right\}\right) .
\end{aligned}
$$

Utilizando os resultados do lema 4.1, temos que o resultado segue.

Teorema 4.11. [Belzunce et al. (2007)] Seja $X=(X, Y)$ um vetor aleatório contínuo com cópula $C$. Então a probabilidade acumulada na região lateral de ordem $p$ e direção $\varepsilon, P\left(\boldsymbol{X} \in L_{\boldsymbol{X}}(p, \varepsilon)\right)$, depende somente da cópula $C$.

A figura abaixo ilustra as curvas quantis e a região central descrita acima para o exemplo 4.3.

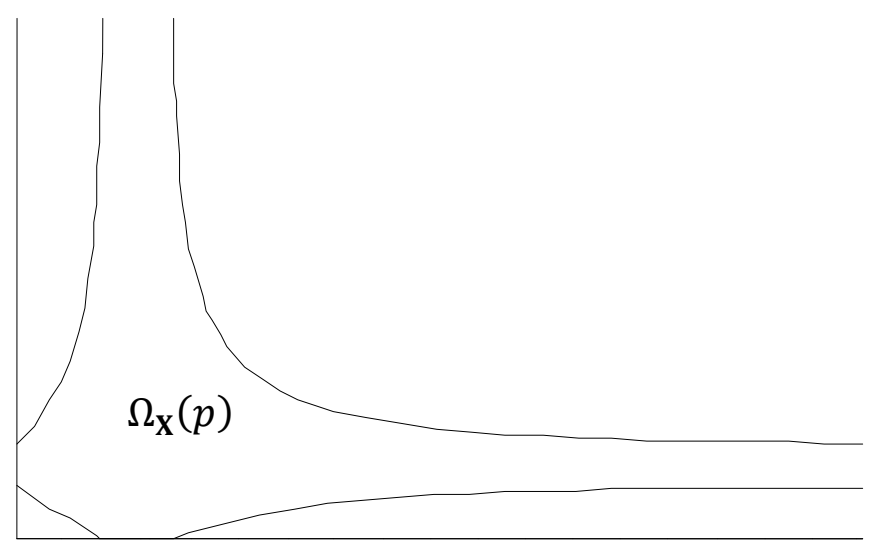

Figura 4.2: A região central. 


\subsubsection{Aplicações dos Quantis Bivariados à Mensuração de Não-Permutabilidade e Assimetria Radial}

Nesta subseção o conceito de curva quantil bivariada é utilizado para definir medidas para analisar a permutabilidade e a assimetria radial da estrutura de dependência dos dados. Para isso são desenvolvidos os resultados a seguir.

Teorema 4.12. Seja $\boldsymbol{X}=(X, Y)$ um vetor aleatório contínuo com cópula $C$ e sejam as cópulas $C^{--}, C^{-+}, C^{+-}$e $C^{++}$como definidas abaixo:

$$
\begin{aligned}
& C^{--}(u, v)=C(u, v) \\
& C^{-+}(u, v)=u-C(u, 1-v) \\
& C^{+-}(u, v)=v-C(1-u, v) \\
& C^{++}(u, v)=u+v-1+C(1-u, 1-v)
\end{aligned}
$$

Então são válidos os seguintes resultados:

1) $P\left(\boldsymbol{X} \in L_{\boldsymbol{X}}\left(p, \varepsilon_{--}\right)\right)=\mu_{C}\left(\left\{(u, v) \in[0,1]^{2}: C(u, v)>p\right\}\right)$,

$$
=\mu_{C^{--}}\left(\left\{(u, v) \in[0,1]^{2}: C^{--}(u, v)>p\right\}\right) ;
$$

2) $P\left(\boldsymbol{X} \in L_{\boldsymbol{X}}\left(p, \varepsilon_{-+}\right)\right)=\mu_{C}\left(\left\{(u, v) \in[0,1]^{2}: u-C(u, v)>p\right\}\right)$,

$$
=\mu_{C^{-+}}\left(\left\{(u, v) \in[0,1]^{2}: C^{-+}(u, v)>p\right\}\right) ;
$$

3) $P\left(\boldsymbol{X} \in L_{\boldsymbol{X}}\left(p, \varepsilon_{+-}\right)\right)=\mu_{C}\left(\left\{(u, v) \in[0,1]^{2}: v-C(u, v)>p\right\}\right)$,

$$
=\mu_{C^{+-}}\left(\left\{(u, v) \in[0,1]^{2}: C^{+-}(u, v)>p\right\}\right) \text {; }
$$

4) $P\left(\boldsymbol{X} \in L_{\boldsymbol{X}}\left(p, \varepsilon_{++}\right)\right)=\mu_{C}\left(\left\{(u, v) \in[0,1]^{2}: 1-u-v+C(u, v)>p\right\}\right)$,

$$
=\mu_{C^{++}}\left(\left\{(u, v) \in[0,1]^{2}: C^{++}(u, v)>p\right\}\right) .
$$

Prova: Pela analogia de 1), 2), 3) e 4), basta demonstrar um desses resultados. Analisando 2), temos que

$$
\begin{aligned}
\mu_{C^{-+}}\left(\left\{(u, v) \in[0,1]^{2}: C^{-+}(u, v)>p\right\}\right) & =\iiint_{\left\{(u, v) \in[0,1]^{2}: C^{-+}(u, v)>p\right\}} d C^{-+}(u, v) \\
& =\int_{\left\{(u, v) \in[0,1]^{2}: u-C(u, 1-v)>p\right\}} d[u-C(u, 1-v)] \\
& =\int_{\left\{(u, w) \in[0,1]^{2}: u-C(u, w)>p\right\}} d C(u, w) \\
& =\mu_{C}\left(\left\{(u, v) \in[0,1]^{2}: u-C(u, v)>p\right\}\right) .
\end{aligned}
$$


Corolário 4.3. Colocando as probabilidades associadas às regiões laterais em termos da Transformada Integral de Probabilidade bivariada, sendo denotada por $(C \mid C)(p)$, temos que:

(i) $\quad P\left(\boldsymbol{X} \in L_{\boldsymbol{X}}\left(p, \varepsilon_{--}\right)\right)=1-\left(C^{--} \mid C^{--}\right)(p)$.

(ii) $\quad P\left(\boldsymbol{X} \in L_{X}\left(p, \varepsilon_{-+}\right)\right)=1-\left(C^{-+} \mid C^{-+}\right)(p)$.

(iii) $\quad P\left(\boldsymbol{X} \in L_{\boldsymbol{X}}\left(p, \varepsilon_{+-}\right)\right)=1-\left(C^{+-} \mid C^{+-}\right)(p)$.

(iv) $\quad P\left(\boldsymbol{X} \in L_{\boldsymbol{X}}\left(p, \varepsilon_{++}\right)\right)=1-\left(C^{++} \mid C^{++}\right)(p)$.

A função $(C \mid C)(p)$ é também denominada função distribuição de Kendall e, portanto, as probabilidades associadas às regiões laterais dadas em (i)-(iv) acima são funções de sobrevivência de Kendall. Para maiores detalhes sobre funções distribuição de Kendall, ver Nelsen et al. (2003).

Os teoremas a seguir mostram a relação entre as massas nas regiões laterais quando existem permutabilidade e simetria radial na cópula subjacente ao vetor aleatório.

Teorema 4.13. Seja $X=(X, Y)$ um vetor aleatório contínuo com cópula $C$ e sejam as cópulas $C^{-+}$e $C^{+-}$como definidas anteriormente. Então se $C(u, v)=C(v, u)$, para todo $(u, v)$ em $[0,1]^{2}$, temos que $\left(C^{-+} \mid C^{-+}\right)(p)=\left(C^{+-} \mid C^{+-}\right)(p)$, para todo $p \in[0,1]$.

Prova: Como $C(u, v)=C(v, u)$, para todo $(u, v)$ em $[0,1]^{2}$, temos que

$$
\begin{aligned}
\left(C^{-+} \mid C^{-+}\right)(p) & =\mu_{C}\left(\left\{(u, v) \in[0,1]^{2}: u-C(u, v)>p\right\}\right) ; \\
& =\iint_{\left\{(u, v) \in[0,1]^{2}: u-C(u, v)>p\right\}} d C(u, v) ; \\
& =\iint_{\left\{(x, y) \in[0,1]^{2}: y-C(y, x)>p\right\}} d C(y, x) ; \\
& =\iint_{\left\{(x, y) \in[0,1]^{2}: y-C(x, y)>p\right\}} d C(x, y) ; \\
& =\mu_{C}\left(\left\{(u, v) \in[0,1]^{2}: v-C(u, v)>p\right\}\right)=\left(C^{+-} \mid C^{+-}\right)(p) .
\end{aligned}
$$

Portanto $\left(C^{-+} \mid C^{-+}\right)(p)=\left(C^{+-} \mid C^{+-}\right)(p)$, para todo $p \in[0,1]$.

Teorema 4.14. Seja $\mathbf{X}=(X, Y)$ um vetor aleatório contínuo com cópula $C$ e sejam as cópulas $C^{--}, C^{-+}, C^{+-}$e $C^{++}$como definidas anteriormente. Se para todo $(u, v)$ em $[0,1]^{2}$ é válido que

$$
C(u, v)=1-(1-u)-(1-v)+C(1-u, 1-v),
$$

Então temos que $\left(C^{--} \mid C^{--}\right)(p)=\left(C^{++} \mid C^{++}\right)(p)$ e $\left(C^{-+} \mid C^{-+}\right)(p)=\left(C^{+-} \mid C^{+-}\right)(p)$, para todo $p \in[0,1]$. 
Prova: A demonstração da igualdade de $\left(C^{--} \mid C^{--}\right)(p)$ e $\left(C^{++} \mid C^{++}\right)(p)$ é imediata, bastando notar que $C(u, v)=C^{-}(u, v)$ e $\hat{C}(u, v)=C^{++}(u, v)$, para todo $(u, v)$ em $[0,1]^{2}$, onde $\hat{C}(u, v)$ representa a cópula de sobrevivência associada a $C(u, v)$.

Analisando a igualdade de $\left(C^{-+} \mid C^{-+}\right)(p)$ e $\left(C^{+-} \mid C^{+-}\right)(p)$, temos que

$$
C(u, v)=\hat{C}(u, v) \Leftrightarrow C^{-+}(u, v)=C^{+-}(u, v),
$$

para todo $(u, v)$ em $[0,1]^{2}$. Como $C^{+-}(u, v)$ é a cópula de sobrevivência de $C^{-+}(u, v)$, temos que o resultado segue.

Teorema 4.15. Seja $X=(X, Y)$ um vetor aleatório contínuo com cópula $C$ e coeficiente Kendall $\tau_{C}$ e sejam $C^{--}, C^{-+}, C^{+-}$e $C^{++}$cópulas como definidas anteriormente. Então as seguintes igualdades são válidas:

$$
\begin{aligned}
& \int_{0}^{1}\left(C^{--} \mid C^{--}\right)(p) d p=\int_{0}^{1}\left(C^{++} \mid C^{++}\right)(p) d p=\frac{3-\tau_{C}}{4} \mathrm{e} \\
& \int_{0}^{1}\left(C^{-+} \mid C^{-+}\right)(p) d p=\int_{0}^{1}\left(C^{+-} \mid C^{+-}\right)(p) d p=\frac{3+\tau_{C}}{4} .
\end{aligned}
$$

Prova: Temos que o coeficiente Kendall $\tau$ associado à cópula $C, \tau_{C}$, pode ser expresso como

$$
\tau_{C}=3-4 \int_{0}^{1}(C \mid C)(p) d p=4 \int_{0}^{1} \int_{0}^{1} C(u, v) d C(u, v)-1 .
$$

Uma vez que $C^{--}=C$, segue que $\tau_{C^{--}}=\tau_{C}$. Analisando $C^{++}$, temos que

$$
\begin{aligned}
\tau_{C^{++}} & =4 \int_{0}^{1} \int_{0}^{1} C^{++}(u, v) d C^{++}(u, v)-1 \\
& =4 \int_{0}^{1} \int_{0}^{1}[u+v-1+C(1-u, 1-v)] d C(1-u, 1-v)-1 \\
& =4 \int_{0}^{1} \int_{0}^{1} C(x, y) d C(x, y)-1 \\
& =\tau_{C} .
\end{aligned}
$$

Uma vez que $C^{++}$é a cópula de sobrevivência associada a $C^{--}$, temos que para qualquer cópula $C$ e sua cópula de sobrevivência associada $\hat{C}$, é válido que $\tau_{C}=\tau_{C}$.

Analisando $C^{-+}$, temos que $\tau_{C^{-+}}=\tau_{C^{+-}}$, uma vez que $C^{-+}$é a cópula de sobrevivência associada a $C^{+-}$. 
Adicionalmente, temos que

$$
\begin{aligned}
\tau_{C^{-+}} & =4 \int_{0}^{1} \int_{0}^{1} C^{-+}(u, v) d C^{-+}(u, v)-1 \\
& =4 \int_{0}^{1} \int_{0}^{1}[u-C(u, 1-v)] d C(u, 1-v)-1 \\
& =-4 \int_{0}^{1} \int_{0}^{1} C(x, y) d C(x, y)+1 \\
& =-\tau_{C} .
\end{aligned}
$$

Portanto o resultado segue.

Com base nestes resultados propomos as seguintes medidas, baseada nos quantis bivariados, de assimetria radial e não-permutabilidade:

Definição 4.8. A medida de assimetria radial da cópula $C$ baseada nos quantis bivariados do vetor aleatório contínuo $(X, Y)$ que tem $C$ como estrutura de dependência é dada por:

onde

$$
\Sigma_{C}=\operatorname{Max}\left(\Sigma_{1}, \Sigma_{2}\right)
$$

$$
\begin{aligned}
& \Sigma_{1}=3 \int_{0}^{1}\left[\left(C^{++} \mid C^{++}\right)(p)-\left(C^{--} \mid C^{--}\right)(p)\right]^{2} d p \mathrm{e} \\
& \Sigma_{2}=3 \int_{0}^{1}\left[\left(C^{+-} \mid C^{+-}\right)(p)-\left(C^{-+} \mid C^{-+}\right)(p)\right]^{2} d p .
\end{aligned}
$$

O caso onde $\Sigma_{C}=0$ corresponde à simetria radial da cópula $C$ e, portanto, à simetria radial da estrutura de dependência de $(X, Y)$.

Definição 4.9. A medida de não-permutabilidade da cópula $C$ baseada nos quantis bivariados do vetor aleatório contínuo $(X, Y)$ que tem $C$ como estrutura de dependência é dada por:

$$
\Pi_{C}=3 \int_{0}^{1}\left[\left(C^{+-} \mid C^{+-}\right)(p)-\left(C^{-+} \mid C^{-+}\right)(p)\right]^{2} d p
$$

O caso onde $\Pi_{C}=0$ corresponde à permutabilidade da cópula $C$ e, portanto, à simetria da estrutura de dependência de $(X, Y)$. 


\section{Observações:}

1) Os fatores quadrados nos integrandos de $\Sigma_{1}, \Sigma_{2}$ e $\Pi_{C}$ são aplicados às definições das medidas $\Sigma_{C}$ e $\Pi_{C}$ em vista do teorema 4.15, uma vez que de outra forma essas medidas seriam iguais a zero para qualquer cópula $C$.

2) O multiplicador 3 nas definições de $\Sigma_{1}$ e $\Sigma_{2}$ é uma constante normalizadora, uma vez que para qualquer cópula $C$ temos que $p \leq(C \mid C)(p) \leq 1$, e, portanto, para quaisquer duas cópulas $C_{a}$ e $C_{b}$ temos que $\int_{0}^{1}\left[\left(C_{a} \mid C_{a}\right)(p)-\left(C_{b} \mid C_{b}\right)(p)\right]^{2} d p \leq \frac{1}{3}$, resultando assim que $\Sigma_{C}, \Pi_{C} \in[0,1)$.

3) A motivação para o uso da função $M a x$ na definição da medida $\Sigma_{C}$ é o resultado do teorema 4.14, que estabelece que no caso onde $C$ é radialmente simétrica, ambos $\Sigma_{1}$ e $\Sigma_{2}$ devem ser iguais a zero.

Os exemplos a seguir mostram a aplicação dessas medidas para alguns casos especiais de cópulas.

Exemplo 4.4. [Família Fréchet-Mardia] Seja a cópula formada pela média ponderada dos casos de dependência total negativa, independência e dependência total positiva, $W(u, v)=\operatorname{Max}(u+v-1,0), \Pi(u, v)=u v$ e $M(u, v)=\operatorname{Min}(u, v)$, respectivamente, dada por:

$$
C_{\alpha, \beta}(u, v)=\alpha W(u, v)+(1-\alpha-\beta) \Pi(u, v)+\beta M(u, v), \quad \alpha, \beta \in[0,1] \text { e } \alpha+\beta \leq 1
$$

Temos que $C_{\alpha, \beta}(u, v)=C_{\alpha, \beta}(v, u)$ e pela simetria radial das cópulas $W$, $\Pi$ e $M$, temos que $C_{\alpha, \beta}(u, v)=\hat{C}_{\alpha, \beta}(u, v)$, e, portanto, $\Pi_{C}=\Sigma_{C}=0$.

Exemplo 4.5. Seja a cópula $C(u, v)$ dada por:

$$
C(u, v)=\operatorname{Min}\left(u, v,\left(u-\frac{2}{3}\right)^{+}+\left(v-\frac{1}{3}\right)^{+}\right)
$$

Esse caso corresponde a uma cópula maximalmente não-permutável e maximalmente radialmente assimétrica segundo o critério proposto em Nelsen (2007).

Temos que:

$$
\begin{gathered}
\left(C^{--} \mid C^{--}\right)(p)=\operatorname{Min}\left(p, \frac{1}{3}\right)+\operatorname{Min}\left(p, \frac{2}{3}\right)=\left(C^{++} \mid C^{++}\right)(p), \\
\left(C^{+-} \mid C^{+-}\right)(p)=\frac{1+2 \mathbb{1}\left(p \geq \frac{1}{3}\right)}{3} \text { e }\left(C^{-+} \mid C^{-+}\right)(p)=\frac{2+\mathbb{1}\left(p \geq \frac{2}{3}\right)}{3} .
\end{gathered}
$$

Calculando as integrais obtém-se que $\Sigma_{1}=0$ e $\Sigma_{2}=\frac{2}{9}$. Portanto, $\Sigma_{C}=\frac{2}{9}$ e $\Pi_{C}=\frac{2}{9}$. 
Neste caso a medida de não-permutabilidade, $\Pi_{C}$, e a medida de assimetria radial, $\Sigma_{C}$, identificaram de maneira correta as assimetrias da cópula $C$.

A seguir temos um contra-exemplo onde a cópula é não-permutável, mas com $\Sigma_{C}=0$.

Exemplo 4.6. Seja a cópula $C^{*}(u, v)$ um "Shuffle of $\mathrm{M}^{\prime}$, ver Mikusinki et al. (1992), com o suporte representado na seguinte figura:

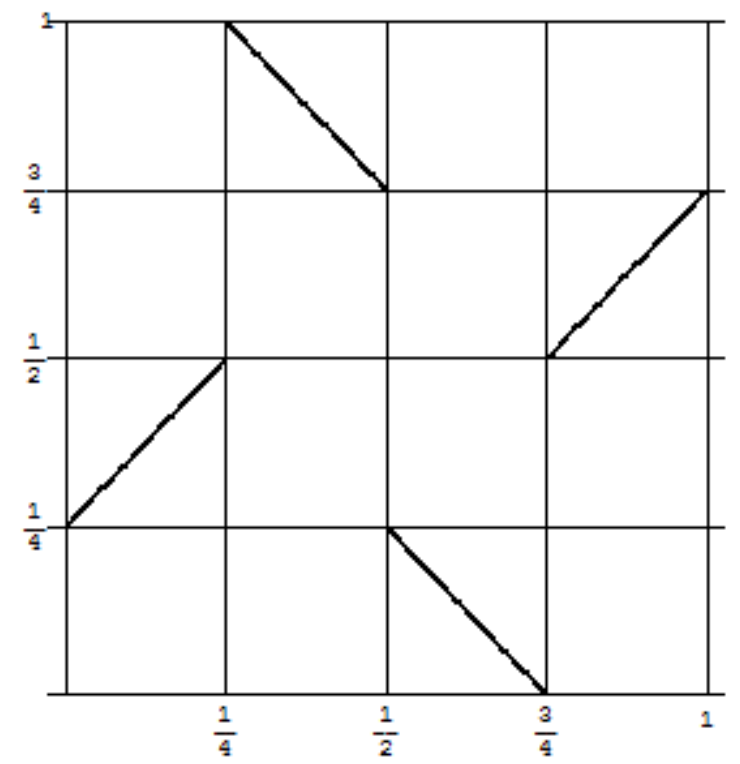

Figura 4.3: Exemplo de cópula $C(u, v)$ tal que $C(u, v) \neq C(v, u)$, mas com $\Sigma_{C}=0$.

É fácil verificar que $C(u, v) \neq C(v, u)$. No entanto, a cópula $C(u, v)$ é radialmente simétrica e pelo teorema 4.14 temos que $\left(C^{-+} \mid C^{-+}\right)(p)=\left(C^{+-} \mid C^{+-}\right)(p)$, para todo $p \in[0,1]$, e portanto $\Sigma_{C}=0$.

\subsubsection{Versões Empíricas e Testes de Hipóteses}

O próximo teorema apresenta as versões amostrais das medidas de não-permutabilidade e assimetria radial apresentadas na subseção anterior.

Teorema 4.16. Seja $\breve{C}\left(\frac{i}{n}, \frac{j}{n}\right)$ a cópula empírica do vetor de observações $\left\{\left(x_{k}, y_{k}\right)\right\}_{k=1}^{n}$ do vetor aleatório contínuo $(X, Y)$, dada por:

$$
\breve{C}\left(\frac{i}{n}, \frac{j}{n}\right)=\frac{1}{n}\left\{\# \text { de pontos }\left(x_{k}, y_{k}\right) \text { tais que } x_{k} \leq x_{(i)} \text { e } y_{k} \leq y_{(j)}\right\}
$$


onde $(i)$ denota a $i$-ésima estatística de ordem e seja $(\breve{C} \mid \breve{C})(p)$ a Transformada Integral de Probabilidade bivariada empírica dada por:

$$
(\breve{C} \mid \breve{C})(p)=\frac{1}{n}\left\{\# \text { de pares }\left(x_{k}, y_{k}\right) \text { cujos "ranks" }(i, j) \text { satisfazem } \breve{C}\left(\frac{i}{n}, \frac{j}{n}\right) \leq p\right\} \text {. }
$$

Temos que as versões amostrais de $\Pi_{C}$ e $\Sigma_{C}$, para uma amostra de tamanho $n$ de pares $\left(x_{k}, y_{k}\right)$, são dadas por:

$$
\begin{gathered}
\breve{\Pi}_{C}=\frac{3}{n} \sum\left[\frac{j}{n}-\breve{C}\left(\frac{n-i}{n}, \frac{j}{n}\right)-\frac{i}{n}+\breve{C}\left(\frac{i}{n}, \frac{n-j}{n}\right)\right]^{2} \mathrm{e} \\
\breve{\Sigma}_{C}=\operatorname{Max}\left(\breve{\Sigma}_{1}, \breve{\Sigma}_{2}\right),
\end{gathered}
$$

onde

$$
\begin{aligned}
& \breve{\Sigma}_{1}=\frac{3}{n} \sum\left[1-\frac{i}{n}-\frac{j}{n}+\breve{C}\left(\frac{i}{n}, \frac{j}{n}\right)-\breve{C}\left(\frac{n-i}{n}, \frac{n-j}{n}\right)\right]^{2} \mathrm{e} \\
& \breve{\Sigma}_{2}=\frac{3}{n} \sum\left[\frac{j}{n}-\breve{C}\left(\frac{n-i}{n}, \frac{j}{n}\right)-\frac{i}{n}+\breve{C}\left(\frac{i}{n}, \frac{n-j}{n}\right)\right]^{2}
\end{aligned}
$$

onde os somatórios são tomados sobre todos os pontos da amostra.

Prova: Sejam $\left(C^{\prime} \mid C^{\prime}\right)(p)$ e $\left(C^{\prime \prime} \mid C^{\prime \prime}\right)(p)$ duas Transformadas Integrais de Probabilidade bivariadas empíricas. Como $\left(C^{\prime} \mid C^{\prime}\right)(p)$ e $\left(C^{\prime \prime} \mid C^{\prime \prime}\right)(p)$ são funções escada, temos que

$$
\int_{0}^{1}\left[\left(C^{\prime} \mid C^{\prime}\right)(p)-\left(C^{\prime \prime} \mid C^{\prime \prime}\right)(p)\right]^{2} d p=\frac{1}{n} \sum_{m=1}^{n-1}\left[\left(C^{\prime} \mid C^{\prime}\right)\left(\frac{m}{n}\right)-\left(C^{\prime \prime} \mid C^{\prime \prime}\right)\left(\frac{m}{n}\right)\right]^{2} .
$$

Mas neste somatório um ponto amostral cujos "ranks" são $(i, j)$ é contado $n-m$ vezes quando $C^{\prime}\left(\frac{i}{n}, \frac{j}{n}\right)=\frac{m}{n}$, e portanto

$$
\sum_{m=1}^{n-1}\left[\left(C^{\prime} \mid C^{\prime}\right)\left(\frac{m}{n}\right)-\left(C^{\prime \prime} \mid C^{\prime \prime}\right)\left(\frac{m}{n}\right)\right]^{2}=\sum\left[C^{\prime}\left(\frac{i}{n}, \frac{j}{n}\right)-C^{\prime \prime}\left(\frac{i}{n}, \frac{j}{n}\right)\right]^{2}
$$

onde o somatório da direita é tomado sobre todos os pontos da amostra. Temos então que

$$
\int_{0}^{1}\left[\left(C^{\prime} \mid C^{\prime}\right)(p)-\left(C^{\prime \prime} \mid C^{\prime \prime}\right)(p)\right]^{2} d p=\frac{1}{n} \sum\left[C^{\prime}\left(\frac{i}{n}, \frac{j}{n}\right)-C^{\prime \prime}\left(\frac{i}{n}, \frac{j}{n}\right)\right]^{2} .
$$

Aplicando as definições de $C^{--}, C^{++}, C^{+-}$e $C^{-+}$temos que o resultado segue. 
É interessante notar o caráter intuitivo das versões amostrais das medidas definidas acima, indicando que as mesmas são funções da distância entre as versões empíricas das cópulas $C^{--}$e $C^{++}$e entre $C^{-+}$e $C^{+-}$, no caso da assimetria radial, e entre $C^{-+}$e $C^{+-}$no caso de nãopermutabilidade.

A seguir são formulados testes de hipóteses, a partir dos resultados desenvolvidos anteriormente, para verificação da presença de não-permutabilidade e de assimetria radial.

Definição 4.10. Sejam $\left\{x_{1 k}, y_{1 k}\right\}_{k=1}^{n}$ e $\left\{x_{2 k}, y_{2 k}\right\}_{k=1}^{m}$ duas amostras do vetor aleatório contínuo $(X, Y)$, de tamanho $n$ e $m$ respectivamente, e sejam $H_{n}(x, y)$ e $H_{m}(x, y)$ as funções distribuição bivariadas empíricas definidas pelas amostras $\left\{x_{1 k}, y_{1 k}\right\}_{k=1}^{n}$ e $\left\{x_{2 k}, y_{2 k}\right\}_{k=1}^{m}$ respectivamente, com marginais $F_{n, X}(x), F_{m, X}(x), G_{n, Y}(y)$ e $G_{m, Y}(y)$ em $X$ e $Y$ respectivamente.

Adicionalmente, sejam as seguintes variáveis:

$T_{n}=F_{n, X}\left(x_{1 k}\right)-H_{n}\left(x_{1 k}, y_{1 k}\right)$,

$T_{m}=G_{m, Y}\left(y_{2 k}\right)-H_{m}\left(x_{2 k}, y_{2 k}\right)$,

definidas em $\left\{x_{1 k}, y_{1 k}\right\}_{k=1}^{n}$ e $\left\{x_{2 k}, y_{2 k}\right\}_{k=1}^{m}$ e com funções distribuição $F_{T_{n}}(p)$ e $F_{T_{m}}(p)$ respectivamente, para $p \in(0,1)$.

O teste de hipótese de nível de significância $\alpha$ para verificação de permutabilidade, com base nos quantis bivariados, é definido da seguinte forma:

$\mathcal{H}_{0}: F_{T_{n}}(p)=F_{T_{m}}(p)$, para todo $p \in(0,1)$,

contra

$\mathcal{H}_{1}: F_{T_{n}}(p) \neq F_{T_{m}}(p)$, para ao menos um valor de $p \in(0,1)$.

No caso de assimetria radial a estrutura do teste é semelhante, sendo definido a seguir:

Definição 4.11. Sejam $\left\{x_{1 k}, y_{1 k}\right\}_{k=1}^{n}$ e $\left\{x_{2 k}, y_{2 k}\right\}_{k=1}^{m}$ duas amostras do vetor aleatório contínuo $(X, Y)$, de tamanho $n$ e $m$ respectivamente, e sejam $H_{n}(x, y)$ e $H_{m}(x, y)$ as funções distribuição bivariadas empíricas definidas pelas amostras $\left\{x_{1 k}, y_{1 k}\right\}_{k=1}^{n}$ e $\left\{x_{2 k}, y_{2 k}\right\}_{k=1}^{m}$ respectivamente, com marginais $F_{n, X}(x), F_{m, X}(x), G_{n, Y}(y)$ e $G_{m, Y}(y)$ em $X$ e $Y$ respectivamente.

Adicionalmente, sejam as seguintes variáveis:

$T_{n, 1}=F_{n, X}\left(x_{1 k}\right)-H_{n}\left(x_{1 k}, y_{1 k}\right)$,

$T_{m, 1}=G_{m, Y}\left(y_{2 k}\right)-H_{m}\left(x_{2 k}, y_{2 k}\right)$,

$T_{n, 2}=H_{n}\left(x_{1 k}, y_{1 k}\right)$,

$T_{m, 2}=1-F_{m, X}\left(x_{2 k}\right)-G_{m, Y}\left(y_{2 k}\right)+H_{m}\left(x_{2 k}, y_{2 k}\right)$, 
sendo $F_{T_{n, 1}}(p)$ e $F_{T_{n, 2}}(p)$ definidas em $\left\{x_{1 k}, y_{1 k}\right\}_{k=1}^{n}, F_{T_{m, 1}}(p)$ e $F_{T_{m, 2}}(p)$ definidas em $\left\{x_{2 k}, y_{2 k}\right\}_{k=1}^{m}$ e com funções distribuição $F_{T_{n, 1}}(p), F_{T_{m, 1}}(p), F_{T_{n, 2}}(p)$ e $F_{T_{m, 2}}(p)$, respectivamente, para $p \in(0,1)$.

O teste de hipótese de nível de significância $\alpha$ para verificação de assimetria radial, com base nos quantis bivariados, é definido da seguinte forma:

$\mathcal{H}_{0}: F_{T_{n, 1}}(p)=F_{T_{m, 1}}(p)$ e $F_{T_{n, 2}}(p)=F_{T_{m, 2}}(p)$, para todo $p \in(0,1)$, contra $\mathcal{H}_{1}: F_{T_{n, 1}}(p) \neq F_{T_{m, 1}}(p)$ ou $F_{T_{n, 2}}(p) \neq F_{T_{m, 2}}(p)$, para ao menos um valor de $p \in(0,1)$.

Uma metodologia consagrada para verificação da validade da hipótese $\mathcal{H}_{0}$ do teste de permutabilidade é o teste de duas amostras de Kolmogorov-Smirnov de nível de significância $\alpha$ para verificação da igualdade de duas funções distribuição. Como a igualdade entre $F_{T_{n, 1}}(p)$ e $F_{T_{m, 1}}(p)$ é independente da igualdade entre $F_{T_{n, 2}}(p)$ e $F_{T_{m, 2}}(p)$, uma abordagem semelhante pode ser utilizada, com as devidas alterações, no teste de assimetria radial.

A idéia do teste é utilizar os seguintes fatos:

1) As funções $\left(C^{-+} \mid C^{-+}\right)(p),\left(C^{+-} \mid C^{+-}\right)(p),\left(C^{--} \mid C^{--}\right)(p)$ e $\left(C^{++} \mid C^{++}\right)(p)$ são funções distribuição das variáveis aleatórias $F(X)-H(X, Y), G(Y)-H(X, Y), H(X, Y)$ e $1-F(X)-G(Y)+H(X, Y)$, respectivamente, onde $F$ e $G$ são as distribuições marginais de $X$ e $Y$ respectivamente e $H$ é a distribuição conjunta.

2) A permutabilidade da cópula $C(u, v)$ implica na igualdade de $\left(C^{-+} \mid C^{-+}\right)(p)$ e $\left(C^{+-} \mid C^{+-}\right)(p)$, conforme o teorema 4.13, e a simetria radial nas igualdades, para todo $p \in(0,1)$ entre $\left(C^{--} \mid C^{--}\right)(p)$ e $\left(C^{++} \mid C^{++}\right)(p)$ e entre $\left(C^{-+} \mid C^{-+}\right)(p)$ e $\left(C^{+-} \mid C^{+-}\right)(p)$, conforme o teorema 4.14 .

Como essas distribuições não são conhecidas, suas versões empíricas são usadas com base no fato bem conhecido que $H_{n}(x, y)$ é um estimador fortemente consistente de $H(x, y)$, isto é, $H_{n}(x, y) \stackrel{q . c .}{\rightarrow} H(x, y)$ quando $n \rightarrow \infty$, e nos seguintes resultados:

Lema 4.2. [Belzunce et al. (2007)] Seja $\left\{X_{k}, Y_{k}\right\}_{k=1}^{n}$ uma amostra aleatória de um vetor aleatório $(X, Y)$, com função distribuição $H(x, y)$ e função distribuição empírica $H_{n}(x, y)$. Considere $X^{\prime}, Y^{\prime}$ uma cópia de $X, Y$ e independentemente distribuído em relação à amostra. Então, quando $n \rightarrow \infty$ temos que

$$
H_{n}\left(X^{\prime}, Y^{\prime}\right) \stackrel{q . c .}{\rightarrow} H\left(X^{\prime}, Y^{\prime}\right)
$$


Corolário 4.4. [Belzunce et al. (2007)] Seja $\left\{X_{k}, Y_{k}\right\}_{k=1}^{n}$ uma amostra aleatória de uma função distribuição $H(x, y)$, então fixando-se o $i$-ésimo componente temos que quando $n \rightarrow \infty$

$$
H_{n}\left(X_{i}, Y_{i}\right) \stackrel{\text { q.c. }}{\rightarrow} H\left(X_{i}, Y_{i}\right)
$$

Dessa forma, apesar do desconhecimento da função distribuição do vetor aleatório $(X, Y)$, os resultados acima garantem que assintoticamente os testes propostos são consistentes.

Utilizando essa abordagem, a verificação da permutabilidade e da simetria radial de vetores aleatórios contínuos bivariados, por exemplo $(X, Y)$, com marginais $F, G$ de $X$ e $Y$, respectivamente, e distribuição conjunta $H$, se resume à comparação de funções distribuição univariadas.

No caso da permutabilidade, são necessárias as comparações das marginais de $X$ e $Y$ e a comparação das distribuições apontadas na definição 4.10. Conforme o teorema 4.1, se houver igualdade dessas distribuições a distribuição conjunta de $(X, Y)$ é permutável.

No caso da simetria radial em torno do ponto $(a, b) \in \mathfrak{R}^{2}$, são necessárias as comparações entre as distribuições marginais de $X-a$ e $a-X$, entre as distribuições marginais de $Y-b$ e $b-Y$ e entre as distribuições apontadas na definição 4.11. Conforme o teorema 4.5, se houver igualdade dessas distribuições a distribuição conjunta de $(X, Y)$ é radialmente simétrica.

\subsubsection{Exemplos de Aplicação}

Nesta subseção aplicamos a medida de não-permutabilidade $\breve{\Pi}_{C}$ e o teste de nãopermutabilidade dado na definição 4.10 a dois conjuntos de dados simulados a partir de cópulas pertencentes à famíla FGM (Farlie-Gumbel-Morgenstern) de cópulas assimétricas. A seguir os resultados obtidos são discutidos.

Seja $(U, V)$ um vetor aleatório com $U$ e $V$ uniformemente distribuídos em $(0,1)$ e com cópula $C_{\alpha, \beta}(u, v)=u v+u v(1-u)(1-v)[\alpha+(\beta-\alpha) v(1-u)]$, com $|\alpha| \leq 1 \mathrm{e}$ $(1 / 2)\left[\alpha-3-\left(9+6 \alpha-3 \alpha^{2}\right)^{1 / 2}\right] \leq \beta \leq 1$. Então $C_{\alpha, \beta}(u, v)$ é não-permutável para $\alpha \neq \beta$. Quando $\alpha=\beta$ temos que $C_{\alpha, \beta}(u, v)$ é a cópula da família FGM de parâmetro $\alpha$.

Simulando 10,000 pares $(U, V)$ com $\alpha=\beta=1$ (permutabilidade de $C_{\alpha, \beta}(u, v)$ ), foram obtidos os seguintes resultados:

- O valor empírico da medida de não-permutabilidade obtido foi $\breve{\Pi}_{C},=6.1 \times 10^{-6}$, em consonância com a permutabilidade de $C_{\alpha, \beta}(u, v)$.

- O teste de Kolmogorov-Smirnov de duas amostras para testar a identidade das distribuições entre $\left(C^{-+} \mid C^{-+}\right)(p)$ e $\left(C^{+-} \mid C^{+-}\right)(p)$ obteve um $p$-valor de 0.3305 sob a hipótese nula de igualdade das distribuições, indicando corretamente que $C_{\alpha, \beta}(u, v)$, com $\alpha=\beta=1$, é permutável. 
- Adicionalmente obtive-se que $\breve{\tau}_{C^{-+}}=-0.2265$ e $\breve{\tau}_{C^{+-}}=-0.2136$, em concordância com os valores teóricos esperados de $\tau_{C^{+-}}=\tau_{C^{-+}}=-\tau_{C}=-\frac{2}{9}$.

Temos os seguintes gráficos relativos a este exemplo:

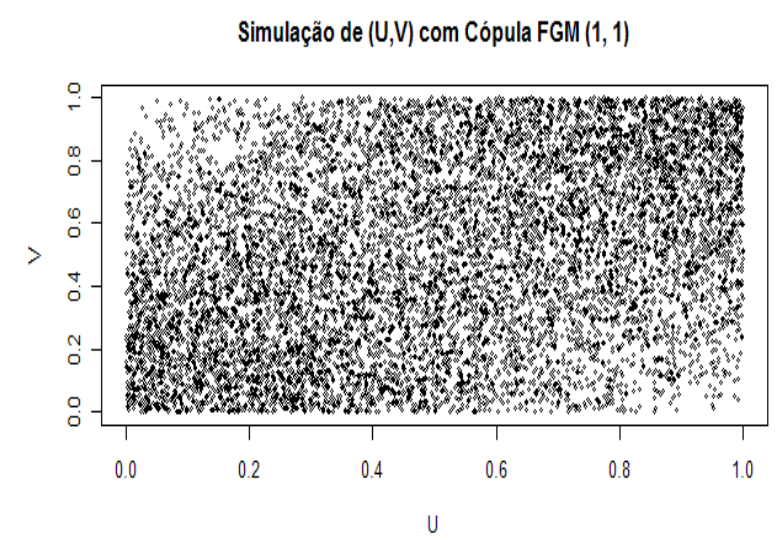

Histograma de (C-+|C-+)

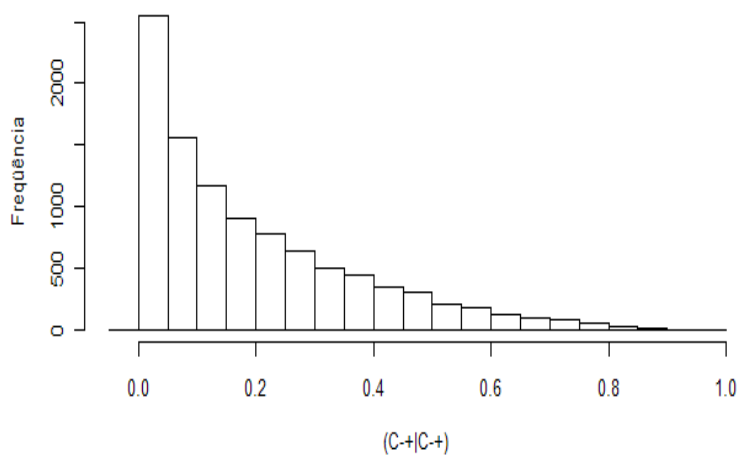

QQ-Plot (C-+|C-+) $\times(\mathrm{C}+-\mid \mathrm{C}+-)$

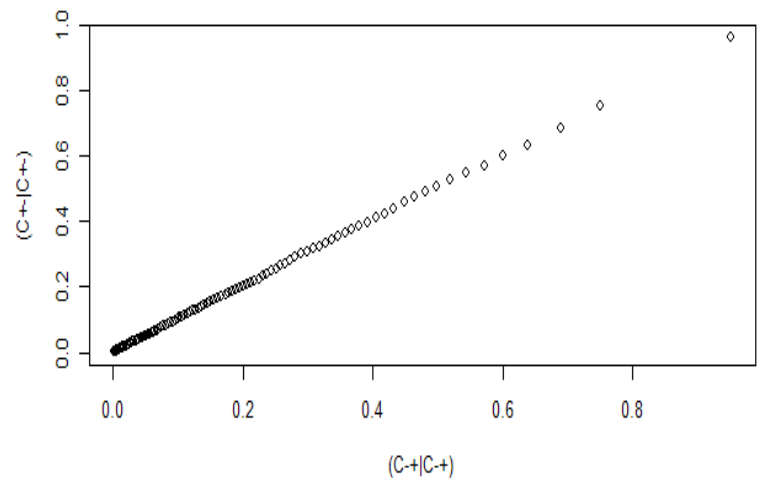

Histograma de $(\mathrm{C}+-\mid \mathrm{C}+-)$

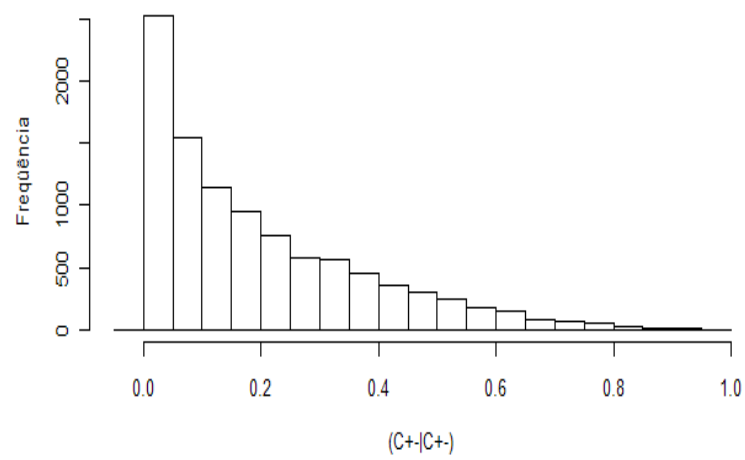

Simulando 10,000 pares $(U, V) \operatorname{com} \alpha=1$ e $\beta=-1-\sqrt{3}$ (não-permutabilidade extrema de $C_{\alpha, \beta}(u, v)$ segundo a medida proposta em Nelsen (2007)), foram obtidos os seguintes resultados:

- Calculando-se o valor empírico da medida de não-permutabilidade, $\breve{\Pi}_{C}$, chegamos a $\breve{\Pi}_{C}=0.003$;

- O teste de Kolmogorov-Smirnov de duas amostras para testar a identidade das distribuições entre $\left(C^{-+} \mid C^{-+}\right)(p)$ e $\left(C^{+-} \mid C^{+-}\right)(p)$ obteve um $p$-valor de $2.2 \times 10^{-16}$ sob a hipótese nula de igualdade das distribuições, indicando corretamente que $C_{\alpha, \beta}(u, v)$, com $\alpha=1$ e $\beta=-1-\sqrt{3}$, não é permutável. 
Temos os seguintes gráficos relativos a este exemplo:
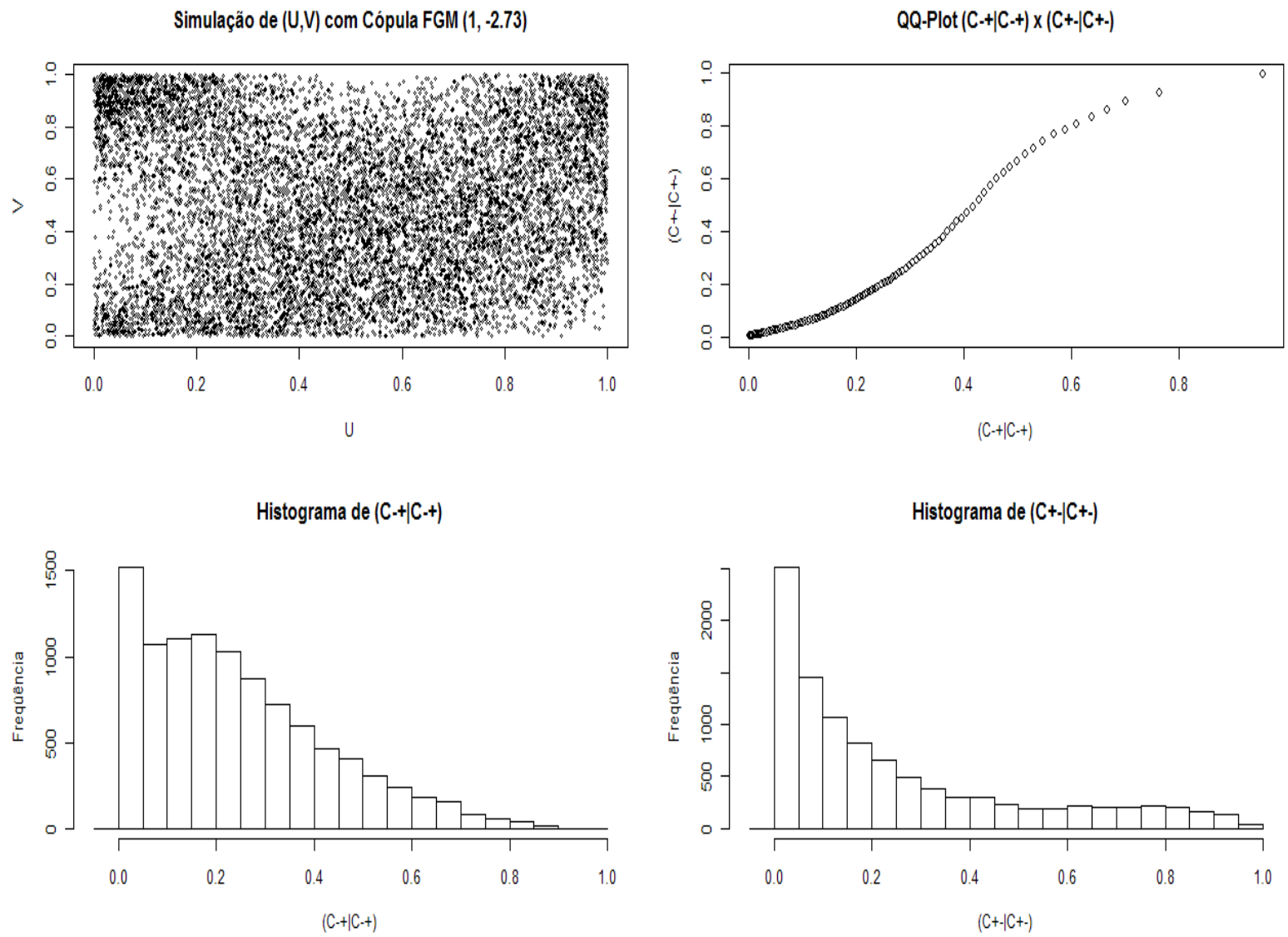

Os exemplos acima mostram que o teste proposto para identificação de nãopermutabilidade baseada nos quantis bivariados apontou corretamente se a cópula em questão era ou não permutável em ambos os casos. Uma análise mais abrangente, em termos de se aplicar esta metodologia a outras famílias de cópulas e de analisar o poder dos testes propostos, são um caminho promissor a ser explorado. 


\section{Conclusão e Pesquisas Futuras}

Nesta tese propusemos novas medidas para quantificar o fenômeno da dependência local e desenvolvemos resultados a respeito de estruturas de dependência assimétrica. As contribuições mais importantes foram:

1) O desenvolvimento das versões locais das medidas Kendall $\tau$ e Spearman $\rho$ e das propriedades que envolvem essas medidas. Algumas aplicações dessas medidas a famílias específicas de cópulas foram implementadas;

2) A obtenção dos limites máximos para o grau de não-associatividade e não-bi-simetria que cópulas bivariadas podem atingir segundo o critério de máxima distância modular e a identificação das famílias de cópulas que atingem esses limites;

3) Uma medida de não-permutabilidade baseada nos coeficientes de correlação condicionais foi proposta e aplicada às distribuições normal simétrica e assimétrica. Contra-exemplos interessantes foram construídos e resultados originais a respeito desses coeficientes foram obtidos. Esses resultados foram apresentados no "28th International Workshop on Bayesian Inference and Maximum Entropy Methods in Science and Engineering" e serão publicados nos "proceedings" deste evento;

4) Foram propostas medidas que avaliassem os graus de não-permutabilidade e assimetria radial presente na estrutura de dependência de vetores contínuos bivariados aleatórios através da ótica das curvas quantis bivariadas. As versões empíricas para as mesmas foram desenvolvidas. Finalmente foram elaborados testes de hipóteses não-paramétricos para determinar se as hipóteses de permutabilidade ou simetria radial são comprovadas pelos dados observados. Esses resultados foram apresentados no $18^{\circ}$ SINAPE.

A partir do trabalho desenvolvido nesta tese, o campo de pesquisas futuras que naturalmente se apresenta é o de estudar formas de construir cópulas a partir de mapas de dependência obtidos a partir das medidas de dependência local que foram propostas. Aplicações desses conceitos em finanças e suas implicações no cálculo de risco de portfólios constituem também uma área promissora. Em relação às medidas de assimetria estudadas, uma linha de pesquisa interessante é a de criar medidas que identifiquem de maneira inequívoca a não-permutabilidade presente nos dados, bem como testes de hipóteses a elas associados. No caso das medidas baseadas nos quantis bivariados, a identificação de cópulas que sejam maximalmente não-permutáveis e obtenção de suas propriedades, bem como análises de poder dos testes de hipóteses propostos são um caminho a ser explorado. 


\section{APÊNDICE A1}

Memória de Cálculo dos Coeficientes de Correlação Condicionais $\rho^{+}$e $\rho^{-}$para a proposição

4.2.

Tomando $U=X-Y$, temos que:

$E(U)=\mu_{X}-\mu_{Y}=\mu_{U}$

$\operatorname{Var}(U)=\sigma_{X}^{2}-2 \rho \sigma_{X} \sigma_{Y}+\sigma_{Y}^{2}=\sigma_{U}^{2}$;

$\operatorname{Corr}(X, U)=\frac{\sigma_{X}-\rho \sigma_{Y}}{\sqrt{\sigma_{X}^{2}-2 \rho \sigma_{X} \sigma_{Y}+\sigma_{Y}^{2}}}=\rho_{X, U}$

$(X, U) \sim \mathcal{N}\left(\mu_{X}, \mu_{U}, \sigma_{X}, \sigma_{U}, \rho_{X, U}\right)$

$(Y, U) \sim \mathcal{N}\left(\mu_{Y}, \mu_{U}, \sigma_{Y}, \sigma_{U}, \rho_{Y, U}\right)$

- $\quad$ Cálculo de $E(X \mid X-Y>0)$ e $E(X \mid X-Y \leq 0)$ :

$P(U>0)=\int_{0}^{\infty} f_{U}(u) d u=\int_{0}^{\infty} \frac{e^{-\frac{1}{2}\left(\frac{u-\mu_{U}}{\sigma_{U}}\right)^{2}}}{\sigma_{U} \sqrt{2 \pi}} d u=\Phi\left(\frac{\mu_{U}}{\sigma_{U}}\right)$.

$E(X, U>0)=\int_{-\infty}^{\infty} \int_{0}^{\infty} x f_{X, U}(x, u) d u d x=\int_{0}^{\infty} f_{U}(u) \int_{-\infty}^{\infty} x f_{X \mid U}(x \mid u) d x d u$

$=\int_{0}^{\infty} f_{U}(u)\left[\mu_{X}+\rho_{X, U} \frac{\sigma_{X}}{\sigma_{U}}\left(u-\mu_{U}\right)\right] d u=\mu_{X} \Phi\left(\frac{\mu_{U}}{\sigma_{U}}\right)+\rho_{X, U} \sigma_{X} \phi\left(\frac{\mu_{U}}{\sigma_{U}}\right)$.

Portanto

$E(X \mid X-Y>0)=\mu_{X}+\frac{\rho_{X, U} \sigma_{X} \phi\left(\frac{\mu_{U}}{\sigma_{U}}\right)}{\Phi\left(\frac{\mu_{U}}{\sigma_{U}}\right)}$.

Como $E(X)=E(X \mid U>0) P(U>0)+E(X \mid U \leq 0) P(U \leq 0)$, temos que

$E(X \mid X-Y \leq 0)=\mu_{X}-\frac{\rho_{X, U} \sigma_{X} \phi\left(\frac{\mu_{U}}{\sigma_{U}}\right)}{1-\Phi\left(\frac{\mu_{U}}{\sigma_{U}}\right)}$. 
- $\quad$ Cálculo de $E(Y \mid X-Y>0)$ e $E(Y \mid X-Y \leq 0)$ :

Temos que $E(Y \mid U>0)=E(X \mid U>0)-E(U \mid U>0)$.

Mas

$E(U \mid U>0)=\int_{0}^{\infty} u f_{U}(u) d u=\int_{0}^{\infty} u \frac{e^{-\frac{1}{2}\left(\frac{u-\mu_{U}}{\sigma_{U}}\right)^{2}}}{\sigma_{U} \sqrt{2 \pi}} d u=\mu_{U} \Phi\left(\frac{\mu_{U}}{\sigma_{U}}\right)+\sigma_{U} \phi\left(\frac{\mu_{U}}{\sigma_{U}}\right)$.

Portanto

$E(Y \mid X-Y>0)=\mu_{X}-\mu_{U}+\frac{\left(\rho_{X, U} \sigma_{X}-\sigma_{U}\right) \phi\left(\frac{\mu_{U}}{\sigma_{U}}\right)}{\Phi\left(\frac{\mu_{U}}{\sigma_{U}}\right)}$.

Como $E(Y)=E(Y \mid U>0) P(U>0)+E(Y \mid U \leq 0) P(U \leq 0)$, temos que

$E(Y \mid X-Y \leq 0)=\mu_{X}-\mu_{U}-\frac{\left(\rho_{X, U} \sigma_{X}-\sigma_{U}\right) \phi\left(\frac{\mu_{U}}{\sigma_{U}}\right)}{1-\Phi\left(\frac{\mu_{U}}{\sigma_{U}}\right)}$.

- $\quad$ Cálculo de $E\left(X^{2} \mid X-Y>0\right)$ e $E\left(X^{2} \mid X-Y \leq 0\right)$ :

$$
\begin{aligned}
E\left(X^{2}, U>0\right) & =\int_{-\infty}^{\infty} \int_{0}^{\infty} x^{2} f_{X, U}(x, u) d u d x=\int_{0}^{\infty} f_{U}(u) \int_{-\infty}^{\infty} x^{2} f_{X \mid U}(x \mid u) d x d u \\
& =\int_{0}^{\infty} f_{U}(u)\left\{\sigma_{X}^{2}\left(1-\rho_{X, U}^{2}\right)+\left[\mu_{X}+\rho_{X, U} \frac{\sigma_{X}}{\sigma_{U}}\left(u-\mu_{U}\right)\right]^{2}\right\} d u \\
& =\left(\sigma_{X}^{2}+\mu_{X}^{2}\right) \Phi\left(\frac{\mu_{U}}{\sigma_{U}}\right)+\left(2 \rho_{X, U} \sigma_{X} \mu_{X}-\frac{\rho_{X, U}^{2} \sigma_{X}^{2} \mu_{U}}{\sigma_{U}}\right) \phi\left(\frac{\mu_{U}}{\sigma_{U}}\right) .
\end{aligned}
$$

Como $E\left(X^{2}\right)=E\left(X^{2} \mid U>0\right) P(U>0)+E\left(X^{2} \mid U \leq 0\right) P(U \leq 0)$, temos que

$E\left(X^{2}, U \leq 0\right)=\left(\sigma_{X}^{2}+\mu_{X}^{2}\right)\left(1-\Phi\left(\frac{\mu_{U}}{\sigma_{U}}\right)\right)-\left(2 \rho_{X, U} \sigma_{X} \mu_{X}-\frac{\rho_{X, U}^{2} \sigma_{X}^{2} \mu_{U}}{\sigma_{U}}\right) \phi\left(\frac{\mu_{U}}{\sigma_{U}}\right)$.

Portanto

$\operatorname{Var}(X \mid X-Y>0)=\frac{E\left(X^{2}, U>0\right)}{P(U>0)}-E^{2}(X \mid X-Y>0)$

$$
=\sigma_{X}^{2}-\frac{\rho_{X, U}^{2} \sigma_{X}^{2} \phi\left(\frac{\mu_{U}}{\sigma_{U}}\right)}{\sigma_{U} \Phi\left(\frac{\mu_{U}}{\sigma_{U}}\right)}\left[\mu_{U}+\frac{\sigma_{U} \phi\left(\frac{\mu_{U}}{\sigma_{U}}\right)}{\Phi\left(\frac{\mu_{U}}{\sigma_{U}}\right)}\right]
$$




$$
\begin{aligned}
\operatorname{Var}(X \mid X-Y \leq 0) & =\frac{E\left(X^{2}, U \leq 0\right)}{P(U \leq 0)}-E^{2}(X \mid X-Y \leq 0) \\
& =\sigma_{X}^{2}+\frac{\rho_{X, U}^{2} \sigma_{X}^{2} \phi\left(\frac{\mu_{U}}{\sigma_{U}}\right)}{\sigma_{U}\left(1-\Phi\left(\frac{\mu_{U}}{\sigma_{U}}\right)\right)}\left[\mu_{U}-\frac{\sigma_{U} \phi\left(\frac{\mu_{U}}{\sigma_{U}}\right)}{1-\Phi\left(\frac{\mu_{U}}{\sigma_{U}}\right)}\right]
\end{aligned}
$$

- $\quad$ Cálculo de $E\left(Y^{2} \mid X-Y>0\right)$ e $E\left(Y^{2} \mid X-Y \leq 0\right)$ :

Fazendo uma analogia entre $X$ e $Y$, temos que

$$
\begin{aligned}
& E\left(Y^{2}, U>0\right)=\left(\sigma_{Y}^{2}+\mu_{Y}^{2}\right) \Phi\left(\frac{\mu_{U}}{\sigma_{U}}\right)+\left(2 \rho_{Y, U} \sigma_{Y} \mu_{Y}-\frac{\rho_{Y, U}^{2} \sigma_{Y}^{2} \mu_{U}}{\sigma_{U}}\right) \phi\left(\frac{\mu_{U}}{\sigma_{U}}\right) \\
& E\left(Y^{2}, U \leq 0\right)=\left(\sigma_{Y}^{2}+\mu_{Y}^{2}\right)\left(1-\Phi\left(\frac{\mu_{U}}{\sigma_{U}}\right)\right)-\left(2 \rho_{Y, U} \sigma_{Y} \mu_{Y}-\frac{\rho_{Y, U}^{2} \sigma_{Y}^{2} \mu_{U}}{\sigma_{U}}\right) \phi\left(\frac{\mu_{U}}{\sigma_{U}}\right)
\end{aligned}
$$

e portanto

$$
\begin{aligned}
\operatorname{Var}(Y \mid X-Y>0) & =\frac{E\left(Y^{2}, U>0\right)}{P(U>0)}-E^{2}(Y \mid X-Y>0) \\
& =\sigma_{Y}^{2}-\frac{\rho_{Y, U}^{2} \sigma_{Y}^{2} \phi\left(\frac{\mu_{U}}{\sigma_{U}}\right)}{\sigma_{U} \Phi\left(\frac{\mu_{U}}{\sigma_{U}}\right)}\left[\mu_{U}+\frac{\sigma_{U} \phi\left(\frac{\mu_{U}}{\sigma_{U}}\right)}{\Phi\left(\frac{\mu_{U}}{\sigma_{U}}\right)}\right] \\
\operatorname{Var}(Y \mid X-Y \leq 0)= & \frac{E\left(Y^{2}, U \leq 0\right)}{P(U \leq 0)}-E^{2}(Y \mid X-Y \leq 0) \\
& =\sigma_{Y}^{2}+\frac{\rho_{Y, U}^{2} \sigma_{Y}^{2} \phi\left(\frac{\mu_{U}}{\sigma_{U}}\right)}{\sigma_{U}\left(1-\Phi\left(\frac{\mu_{U}}{\sigma_{U}}\right)\right)}\left[\mu_{U}-\frac{\sigma_{U} \phi\left(\frac{\mu_{U}}{\sigma_{U}}\right)}{1-\Phi\left(\frac{\mu_{U}}{\sigma_{U}}\right)}\right] .
\end{aligned}
$$

Colocando $\rho_{Y, U}, \sigma_{Y}$ e $\mu_{Y}$ em termos de $\rho_{X, U}, \sigma_{X}, \sigma_{U}, \mu_{X}$ e $\mu_{U}$, temos que

$$
\begin{aligned}
& \operatorname{Var}(Y \mid X-Y>0)=\sigma_{X}^{2}-2 \rho_{X, U} \sigma_{X} \sigma_{U}+\sigma_{U}^{2}-\frac{\left(\rho_{X, U} \sigma_{X}-\sigma_{U}\right)^{2} \phi\left(\frac{\mu_{U}}{\sigma_{U}}\right)}{\sigma_{U} \Phi\left(\frac{\mu_{U}}{\sigma_{U}}\right)}\left[\mu_{U}+\frac{\sigma_{U} \phi\left(\frac{\mu_{U}}{\sigma_{U}}\right)}{\Phi\left(\frac{\mu_{U}}{\sigma_{U}}\right)}\right] . \\
& \operatorname{Var}(Y \mid X-Y \leq 0)=\sigma_{X}^{2}-2 \rho_{X, U} \sigma_{X} \sigma_{U}+\sigma_{U}^{2}+\frac{\left(\rho_{X, U} \sigma_{X}-\sigma_{U}\right)^{2} \phi\left(\frac{\mu_{U}}{\sigma_{U}}\right)}{\sigma_{U}\left(1-\Phi\left(\frac{\mu_{U}}{\sigma_{U}}\right)\right)}\left[\mu_{U}-\frac{\sigma_{U} \phi\left(\frac{\mu_{U}}{\sigma_{U}}\right)}{1-\Phi\left(\frac{\mu_{U}}{\sigma_{U}}\right)}\right]
\end{aligned}
$$


- $\quad$ Cálculo de $E(X Y \mid X-Y>0)$ e $E(X Y \mid X-Y \leq 0)$ :

Temos que $E(X Y \mid U>0)=E\left(X^{2} \mid U>0\right)-E(X U \mid U>0)$.

Mas

$$
\begin{aligned}
E(X U, U>0) & =\int_{-\infty}^{\infty} \int_{0}^{\infty} x u f_{X, U}(x, u) d u d x=\int_{0}^{\infty} u f_{U}(u) \int_{-\infty}^{\infty} x f_{X \mid U}(x \mid u) d x d u \\
& =\int_{0}^{\infty} u f_{U}(u)\left[\mu_{X}+\rho_{X, U} \frac{\sigma_{X}}{\sigma_{U}}\left(u-\mu_{U}\right)\right] d u \\
& =\left(\mu_{X} \mu_{U}+\rho_{X, U} \sigma_{X} \sigma_{U}\right) \Phi\left(\frac{\mu_{U}}{\sigma_{U}}\right)+\mu_{X} \sigma_{U} \phi\left(\frac{\mu_{U}}{\sigma_{U}}\right) .
\end{aligned}
$$

Portanto

$$
\begin{aligned}
E(X Y, U>0) & =\left(\sigma_{X}^{2}+\mu_{X}^{2}-\mu_{X} \mu_{U}-\rho_{X, U} \sigma_{X} \sigma_{U}\right) \Phi\left(\frac{\mu_{U}}{\sigma_{U}}\right) \\
& +\left[2 \rho_{X, U} \sigma_{X} \mu_{X}-\rho_{X, U}^{2} \sigma_{X}^{2}\left(\frac{\mu_{U}}{\sigma_{U}}\right)-\mu_{X} \sigma_{U}\right] \phi\left(\frac{\mu_{U}}{\sigma_{U}}\right)
\end{aligned}
$$

Como $E(X Y)=E(X Y, U>0)+E(X Y, U \leq 0)$, temos que

$$
\begin{aligned}
E(X Y, U \leq 0) & =\left(\sigma_{X}^{2}+\mu_{X}^{2}-\mu_{X} \mu_{U}-\rho_{X, U} \sigma_{X} \sigma_{U}\right)\left[1-\Phi\left(\frac{\mu_{U}}{\sigma_{U}}\right)\right] \\
& -\left[2 \rho_{X, U} \sigma_{X} \mu_{X}-\rho_{X, U}^{2} \sigma_{X}^{2}\left(\frac{\mu_{U}}{\sigma_{U}}\right)-\mu_{X} \sigma_{U}\right] \phi\left(\frac{\mu_{U}}{\sigma_{U}}\right) .
\end{aligned}
$$

Dessa forma temos que

$$
\begin{aligned}
& E(X Y \mid X-Y>0) \\
& \quad=\sigma_{X}^{2}+\mu_{X}^{2}-\mu_{X} \mu_{U}-\rho_{X, U} \sigma_{X} \sigma_{U}+\frac{\left[2 \rho_{X, U} \sigma_{X} \mu_{X}-\rho_{X, U}^{2} \sigma_{X}^{2}\left(\frac{\mu_{U}}{\sigma_{U}}\right)-\mu_{X} \sigma_{U}\right] \phi\left(\frac{\mu_{U}}{\sigma_{U}}\right)}{\Phi\left(\frac{\mu_{U}}{\sigma_{U}}\right)} ; \\
& \begin{aligned}
E(X Y \mid X-Y \leq & 0) \\
& =\sigma_{X}^{2}+\mu_{X}^{2}-\mu_{X} \mu_{U}-\rho_{X, U} \sigma_{X} \sigma_{U}-\frac{\left[2 \rho_{X, U} \sigma_{X} \mu_{X}-\rho_{X, U}^{2} \sigma_{X}^{2}\left(\frac{\mu_{U}}{\sigma_{U}}\right)-\mu_{X} \sigma_{U}\right] \phi\left(\frac{\mu_{U}}{\sigma_{U}}\right)}{1-\Phi\left(\frac{\mu_{U}}{\sigma_{U}}\right)} .
\end{aligned}
\end{aligned}
$$




\section{APÊNDICE A2}

Memória de Cálculo dos Coeficientes de Correlação Condicionais $\rho^{+}$e $\rho^{-}$para a proposição 4.3.

Algumas propriedades importantes da distribuição Normal assimétrica bivariada são dadas abaixo:

$E(X)=\left(\frac{2}{\pi}\right)^{\frac{1}{2}} \frac{\left(\alpha^{\prime}+\omega^{\prime} \beta^{\prime}\right) \sqrt{1-\omega^{\prime 2}}}{\sqrt{\left(\alpha^{\prime} \omega^{\prime}+\beta^{\prime}\right)^{2}+\left(1-\omega^{\prime 2}\right)+\left(\alpha^{\prime}+\omega^{\prime} \beta^{\prime}\right)^{2}-2 \omega^{\prime}\left(\alpha^{\prime}+\omega^{\prime} \beta^{\prime}\right)\left(\alpha^{\prime} \omega^{\prime}+\beta^{\prime}\right)}}$.

$E(Y)=\left(\frac{2}{\pi}\right)^{\frac{1}{2}} \frac{\left(\alpha^{\prime} \omega^{\prime}+\beta^{\prime}\right) \sqrt{1-\omega^{\prime 2}}}{\sqrt{\left(\alpha^{\prime} \omega^{\prime}+\beta^{\prime}\right)^{2}+\left(1-\omega^{\prime 2}\right)+\left(\alpha^{\prime}+\omega^{\prime} \beta^{\prime}\right)^{2}-2 \omega^{\prime}\left(\alpha^{\prime}+\omega^{\prime} \beta^{\prime}\right)\left(\alpha^{\prime} \omega^{\prime}+\beta^{\prime}\right)}}$.

$E\left(X^{2}\right)=E\left(Y^{2}\right)=1$.

$E(X Y)=\omega^{\prime}$.

Adicionalmente temos que se $X \sim \mathcal{N}(0,1)$ então

$E(\Phi(u+v X))=\Phi\left(\frac{u}{\sqrt{1+v^{2}}}\right)$.

Para demonstração desses resultados, ver Azzalini e Valle (1996).

Após uma troca de variáveis trivial temos que $(X, V)=\left(X, \frac{X-Y}{\sqrt{2\left(1-\omega^{\prime}\right)}}\right)$ é distribuído segundo uma distribuição Normal assimétrica com os seguintes parâmetros:

$$
\alpha=\alpha^{\prime}+\beta^{\prime} ; \beta=-\beta^{\prime} \sqrt{2\left(1-\omega^{\prime}\right)} ; \omega=\sqrt{\frac{1-\omega^{\prime}}{2}} .
$$

Para a demonstração dos cálculos dos componentes de $\rho^{+}$e $\rho^{-}$serão necessários alguns lemas que são enunciados e demonstrados a seguir.

Lema A2.1. Com as notações usuais e $\alpha, \beta, u \in \Re$ temos que $\int_{0}^{\infty} \phi(x) \Phi(\alpha x+\beta u) d x=\Phi\left(\frac{\beta u}{\sqrt{1+\alpha^{2}}}\right)-\Psi \frac{-\alpha}{\sqrt{1+\alpha^{2}}}\left(\frac{\beta u}{\sqrt{1+\alpha^{2}}}, 0\right)$,

onde $\Psi_{\rho}(x, y)$ representa a distribuição Normal padrão bivariada acumulada de coeficiente de correlação $\rho$ no ponto $(x, y)$.

Prova: Sejam $U, X \sim \mathcal{N}(0,1)$ independentes. Então 


$$
\begin{gathered}
\int_{0}^{\infty} \phi(x) \Phi(\alpha x+\beta u) d x=E(P(U \leq \alpha X+\beta u \mid X=x), X \geq 0)=P(U \leq \alpha X+\beta u, X \geq 0) \\
=P(U-\alpha X \leq \beta u)-P(U-\alpha X \leq \beta u ; X \leq 0) .
\end{gathered}
$$

Mas

$U-\alpha X \sim \mathcal{N}\left(0, \sqrt{1+\alpha^{2}}\right) \mathrm{e}$

$\rho_{X, \frac{U-\alpha X}{\sqrt{1+\alpha^{2}}}}=\frac{-\alpha}{\sqrt{1+\alpha^{2}}}$

Portanto

$\int_{0}^{\infty} \phi(x) \Phi(\alpha x+\beta u) d x=\Phi\left(\frac{\beta u}{\sqrt{1+\alpha^{2}}}\right)-\Psi \frac{-\alpha}{\sqrt{1+\alpha^{2}}}\left(\frac{\beta u}{\sqrt{1+\alpha^{2}}}, 0\right)$.

De onde segue que

$$
\begin{aligned}
P(X-Y>0) & =\int_{-\infty}^{\infty} \int_{0}^{\infty} 2 \phi_{\omega}(x, v) \Phi(\alpha x+\beta v) d v d x \\
& =2 \int_{0}^{\infty} \phi(v) \int_{-\infty}^{\infty} \phi(z) \Phi\left(\alpha \sqrt{1-\omega^{2}} z+(\alpha \omega+\beta) v\right) d z d v \\
& =1-2 \Psi \frac{-(\alpha \omega+\beta)}{\sqrt{1+\alpha^{2}\left(1-\omega^{2}\right)+(\alpha \omega+\beta)^{2}}}(0,0) .
\end{aligned}
$$

Uma generalização importante é a seguinte

$\int_{a}^{b} \phi(x) \Phi(\alpha x+\beta u) d x=\Psi_{\frac{-\alpha}{\sqrt{1+\alpha^{2}}}}\left(\frac{\beta u}{\sqrt{1+\alpha^{2}}}, b\right)-\Psi_{\frac{-\alpha}{\sqrt{1+\alpha^{2}}}}\left(\frac{\beta u}{\sqrt{1+\alpha^{2}}}, a\right)$.

Lema A2.2. Com as notações usuais e $\alpha, \beta, a, b, u \in \mathfrak{R}$ temos que

$$
\begin{aligned}
\int_{a}^{b} x \phi(x) \Phi(\alpha x & +\beta u) d x \\
& =\frac{\alpha e^{\frac{-\beta^{2} u^{2}}{2\left(1+\alpha^{2}\right)}}}{\sqrt{2 \pi}} \frac{\left[\Phi\left(\sqrt{1+\alpha^{2}} b+\frac{\alpha \beta u}{\sqrt{1+\alpha^{2}}}\right)-\Phi\left(\sqrt{1+\alpha^{2}} a+\frac{\alpha \beta u}{\sqrt{1+\alpha^{2}}}\right)\right]}{\sqrt{1+\alpha^{2}}} \\
& -\phi(b) \Phi(\alpha b+\beta u)+\phi(a) \Phi(\alpha a+\beta u) .
\end{aligned}
$$


Prova: Integrando por partes temos que

$$
\int_{a}^{b} x \phi(x) \Phi(\alpha x+\beta u) d x=\alpha \int_{a}^{b} \phi(x) \phi(\alpha x+\beta u) d x-\phi(b) \Phi(\alpha b+\beta u)+\phi(a) \Phi(\alpha a+\beta u) .
$$

Mas

$$
\begin{gathered}
\int_{a}^{b} \phi(x) \phi(\alpha x+\beta u) d x=\frac{\alpha e^{\frac{-\beta^{2} u^{2}}{2\left(1+\alpha^{2}\right)}}}{\sqrt{2 \pi}} \int_{a}^{b} \frac{e^{\frac{-1}{2}\left[\sqrt{1+\alpha^{2}}\left(x+\frac{\alpha \beta u}{\left(1+\alpha^{2}\right)}\right)\right]^{2}}}{\sqrt{2 \pi}} d x \\
=\frac{\alpha e^{\frac{-\beta^{2} u^{2}}{2\left(1+\alpha^{2}\right)}}}{\sqrt{2 \pi}} \frac{\left[\Phi\left(\sqrt{1+\alpha^{2}} b+\frac{\alpha \beta u}{\sqrt{1+\alpha^{2}}}\right)-\Phi\left(\sqrt{1+\alpha^{2}} a+\frac{\alpha \beta u}{\sqrt{1+\alpha^{2}}}\right)\right]}{\sqrt{1+\alpha^{2}}} .
\end{gathered}
$$

\section{Portanto}

$$
\begin{aligned}
\int_{a}^{b} x \phi(x) \Phi(\alpha x & +\beta u) d x \\
& =\frac{\alpha e^{\frac{-\beta^{2} u^{2}}{2\left(1+\alpha^{2}\right)}}\left[\Phi\left(\sqrt{1+\alpha^{2}} b+\frac{\alpha \beta u}{\sqrt{1+\alpha^{2}}}\right)-\Phi\left(\sqrt{1+\alpha^{2}} a+\frac{\alpha \beta u}{\sqrt{1+\alpha^{2}}}\right)\right]}{\sqrt{1+\alpha^{2}}} \\
& -\phi(b) \Phi(\alpha b+\beta u)+\phi(a) \Phi(\alpha a+\beta u) .
\end{aligned}
$$

Temos os seguintes casos especiais:

- $b=\infty, a=-\infty$.

$\int_{-\infty}^{\infty} x \phi(x) \Phi(\alpha x+\beta u) d x=\frac{\alpha e^{\frac{-\beta^{2} u^{2}}{2\left(1+\alpha^{2}\right)}}}{\sqrt{2 \pi} \sqrt{1+\alpha^{2}}}$

- $b=\infty, a=0$.

$$
\int_{0}^{\infty} x \phi(x) \Phi(\alpha x+\beta u) d x=\frac{\alpha e^{\frac{-\beta^{2} u^{2}}{2\left(1+\alpha^{2}\right)}}}{\sqrt{2 \pi}} \frac{\Phi\left(\frac{-\alpha \beta u}{\sqrt{1+\alpha^{2}}}\right)}{\sqrt{1+\alpha^{2}}}+\frac{\Phi(\beta u)}{\sqrt{2 \pi}} .
$$


Lema A2.3. Com as notações usuais e $\alpha, \beta, a, b, u \in \mathfrak{R}$ temos que

$$
\begin{aligned}
\int_{a}^{b} x^{2} \phi(x) \Phi(\alpha x & +\beta u) d x \\
& =\frac{\alpha e^{\frac{-\beta^{2} u^{2}}{\sqrt{2 \pi} \sqrt{\left.1+\alpha^{2}\right)}}}\left\{\frac{\alpha^{2}}{\sqrt{2 \pi}}\left\{\phi\left(\sqrt{1+\alpha^{2}} a+\frac{\alpha \beta u}{\sqrt{1+\alpha^{2}}}\right)-\phi\left(\sqrt{1+\alpha^{2}} b+\frac{\alpha \beta u}{\sqrt{1+\alpha^{2}}}\right)\right]\right.}{\sqrt{1+\alpha^{2}}} \\
& \left.-\frac{\alpha \beta u\left[\Phi\left(\sqrt{1+\alpha^{2}} b+\frac{\alpha \beta u}{\sqrt{1+\alpha^{2}}}\right)-\Phi\left(\sqrt{1+\alpha^{2}} a+\frac{\alpha \beta u}{\sqrt{1+\alpha^{2}}}\right)\right]}{1+\alpha^{2}}\right\} \\
& +\Psi \frac{-\alpha}{\sqrt{1+\alpha^{2}}}\left(\frac{\beta u}{\sqrt{1+\alpha^{2}}}, b\right)-\Psi_{\frac{-\alpha}{\sqrt{1+\alpha^{2}}}}\left(\frac{\beta u}{\sqrt{1+\alpha^{2}}}, a\right)-b \phi(b) \Phi(\alpha b+\beta u) \\
& +a \phi(a) \Phi(\alpha a+\beta u) .
\end{aligned}
$$

Prova: Integrando por partes temos

$$
\begin{aligned}
& \int_{a}^{b} x^{2} \phi(x) \Phi(\alpha x+\beta u) d x \\
& \quad=\alpha \int_{a}^{b} x \phi(x) \phi(\alpha x+\beta u) d x+\int_{a}^{b} \phi(x) \Phi(\alpha x+\beta u) d x-b \phi(b) \Phi(\alpha b+\beta u) \\
& +a \phi(a) \Phi(\alpha a+\beta u) .
\end{aligned}
$$

Mas

$$
\begin{aligned}
& \int_{a}^{b} \phi(x) \Phi(\alpha x+\beta u) d x=\Psi_{\frac{-\alpha}{\sqrt{1+\alpha^{2}}}}\left(\frac{\beta u}{\sqrt{1+\alpha^{2}}}, b\right)-\Psi_{\frac{-\alpha}{\sqrt{1+\alpha^{2}}}}\left(\frac{\beta u}{\sqrt{1+\alpha^{2}}}, a\right) \mathrm{e} \\
& \int_{a}^{b} x \phi(x) \phi(\alpha x+\beta u) d x \\
& =\frac{e^{\frac{-\beta^{2} u^{2}}{2\left(1+\alpha^{2}\right)}}}{\sqrt{2 \pi} \sqrt{1+\alpha^{2}}}\left\{\frac{\left[\phi\left(\sqrt{1+\alpha^{2}} a+\frac{\alpha \beta u}{\sqrt{1+\alpha^{2}}}\right)-\phi\left(\sqrt{1+\alpha^{2}} b+\frac{\alpha \beta u}{\sqrt{1+\alpha^{2}}}\right)\right]}{\sqrt{1+\alpha^{2}}}\right. \\
& \left.-\frac{\alpha \beta u\left[\Phi\left(\sqrt{1+\alpha^{2}} b+\frac{\alpha \beta u}{\sqrt{1+\alpha^{2}}}\right)-\Phi\left(\sqrt{1+\alpha^{2}} a+\frac{\alpha \beta u}{\sqrt{1+\alpha^{2}}}\right)\right]}{1+\alpha^{2}}\right\}
\end{aligned}
$$

de onde o resultado segue. 
Agregando esses termos temos os seguintes casos especiais:

- $b=\infty, a=-\infty$.

$$
\int_{-\infty}^{\infty} x^{2} \phi(x) \Phi(\alpha x+\beta u) d x=-\frac{\alpha^{2} \beta u e^{\frac{-\beta^{2} u^{2}}{2\left(1+\alpha^{2}\right)}}}{\sqrt{2 \pi}\left(1+\alpha^{2}\right)^{\frac{3}{2}}}+\frac{\Phi(\beta u)}{\sqrt{2 \pi}}
$$

- $b=\infty, a=0$.

$$
\begin{aligned}
\int_{0}^{\infty} x^{2} \phi(x) \Phi(\alpha x & +\beta u) d x \\
& =-\frac{\alpha^{2} \beta u e^{\frac{-\beta^{2} u^{2}}{2\left(1+\alpha^{2}\right)}}}{\sqrt{2 \pi}\left(1+\alpha^{2}\right)^{\frac{3}{2}}} \Phi\left(\frac{-\alpha \beta u}{\sqrt{1+\alpha^{2}}}\right)+\frac{\Phi(\beta u)}{\sqrt{2 \pi}}+\frac{\alpha e^{\frac{-\beta^{2} u^{2}}{2}}}{2 \pi\left(1+\alpha^{2}\right)} \\
& -\Psi \frac{-\alpha}{\sqrt{1+\alpha^{2}}}\left(\frac{\beta u}{\sqrt{1+\alpha^{2}}}, 0\right) .
\end{aligned}
$$

Tendo calculado esses resultados auxiliares podemos passar aos cálculos dos componentes de $\rho^{+}$e $\rho^{-}$.

- $\quad$ Cálculo de $E(X \mid X-Y>0)$ e $E(X \mid X-Y \leq 0)$ :

$$
\begin{aligned}
E(X, X-Y> & 0)=E(X, V>0) \\
& =\int_{0}^{\infty} \int_{-\infty}^{\infty} 2 x \phi_{\omega}(x, v) \Phi(\alpha x+\beta v) d x d v \\
& =\int_{0}^{\infty} \phi(v) \int_{-\infty}^{\infty} 2 x \phi_{\omega}(x \mid v) \Phi(\alpha x+\beta v) d x d v \\
& =\int_{0}^{\infty} \phi(v) \int_{-\infty}^{\infty} 2 x \frac{e^{\frac{-(x-\omega v)^{2}}{2\left(1-\omega^{2}\right)}}}{\sqrt{2 \pi} \sqrt{1-\omega^{2}}} \Phi(\alpha x+\beta v) d x d v \\
& =2 \int_{0}^{\infty} \phi(v) \int_{-\infty}^{\infty}\left(\sqrt{1-\omega^{2}} z+\omega v\right) \frac{e^{\frac{-z^{2}}{2}}}{\sqrt{2 \pi}} \Phi\left(\alpha \sqrt{1-\omega^{2}} z+(\alpha \omega+\beta) v\right) d z d v \\
& =\frac{\alpha\left(1-\omega^{2}\right)}{\sqrt{2 \pi} \sqrt{1+\alpha^{2}\left(1-\omega^{2}\right)+(\alpha \omega+\beta)^{2}}} \\
& +\frac{\omega}{\sqrt{2 \pi}}\left[1+\frac{\alpha \omega+\beta}{\sqrt{1+\alpha^{2}\left(1-\omega^{2}\right)+(\alpha \omega+\beta)^{2}}}\right] \\
& =\frac{\omega}{\sqrt{2 \pi}}+\frac{\alpha+\omega \beta}{\sqrt{2 \pi} \sqrt{1+\alpha^{2}\left(1-\omega^{2}\right)+(\alpha \omega+\beta)^{2}}}
\end{aligned}
$$


Como $E(X)=E(X, X-Y>0)+E(X, X-Y \leq 0)$, temos que

$E(X, X-Y \leq 0)=\left(\frac{2}{\pi}\right)^{\frac{1}{2}} \delta_{X}-\left(\frac{\omega}{\sqrt{2 \pi}}+\frac{\alpha+\omega \beta}{\sqrt{2 \pi} \sqrt{1+\alpha^{2}\left(1-\omega^{2}\right)+(\alpha \omega+\beta)^{2}}}\right)$

onde

$$
\begin{aligned}
& \delta_{X}=\frac{\left(\alpha^{\prime}+\omega^{\prime} \beta^{\prime}\right) \sqrt{1-\omega^{\prime 2}}}{\sqrt{\left(\alpha^{\prime} \omega^{\prime}+\beta^{\prime}\right)^{2}+\left(1-\omega^{\prime 2}\right)+\left(\alpha^{\prime}+\omega^{\prime} \beta^{\prime}\right)^{2}-2 \omega^{\prime}\left(\alpha^{\prime}+\omega^{\prime} \beta^{\prime}\right)\left(\alpha^{\prime} \omega^{\prime}+\beta^{\prime}\right)}} \\
& =\frac{2 \omega(\alpha+\omega \beta) \sqrt{1-\omega^{2}}}{\sqrt{\left[\alpha\left(1-2 \omega^{2}\right)-\omega \beta\right]^{2}+4 \omega^{2}\left(1-\omega^{2}\right)+(\alpha+\omega \beta)^{2}-2\left(1-2 \omega^{2}\right)(\alpha+\omega \beta)\left[\alpha\left(1-2 \omega^{2}\right)-\omega \beta\right]}} .
\end{aligned}
$$

- $\quad$ Cálculo de $E(Y \mid X-Y>0)$ e $E(Y \mid X-Y \leq 0)$ :

$$
\begin{aligned}
E(X-Y, X-Y>0)=2 \omega E(V, V>0) \\
=2 \omega \int_{0}^{\infty} \int_{-\infty}^{\infty} 2 v \phi_{\omega}(x, v) \Phi(\alpha x+\beta v) d x d v \\
=4 \omega \int_{0}^{\infty} v \phi(v) \int_{-\infty}^{\infty} \phi_{\omega}(x \mid v) \Phi(\alpha x+\beta v) d x d v \\
=4 \omega \int_{0}^{\infty} v \phi(v) \int_{-\infty}^{\infty} \frac{e^{\frac{-(x-\omega v)^{2}}{2\left(1-\omega^{2}\right)}}}{\sqrt{2 \pi} \sqrt{1-\omega^{2}}} \Phi(\alpha x+\beta v) d x d v \\
=4 \omega \int_{0}^{\infty} v \phi(v) \int_{-\infty}^{\infty} \frac{e^{\frac{-z^{2}}{2}}}{\sqrt{2 \pi}} \Phi\left(\alpha \sqrt{1-\omega^{2}} z+(\alpha \omega+\beta) v\right) d z d v \\
=\frac{2 \omega}{\sqrt{2 \pi}}\left[1+\frac{\alpha \omega+\beta}{\sqrt{1+\alpha^{2}\left(1-\omega^{2}\right)+(\alpha \omega+\beta)^{2}}}\right] .
\end{aligned}
$$

Temos que $E(Y, X-Y>0)=E(X, X-Y>0)-E(X-Y, X-Y>0)$. Portanto,

$E(Y, X-Y>0)=\frac{\alpha\left(1-2 \omega^{2}\right)-\omega \beta}{\sqrt{2 \pi} \sqrt{1+\alpha^{2}\left(1-\omega^{2}\right)+(\alpha \omega+\beta)^{2}}}-\frac{\omega}{\sqrt{2 \pi}}$.

Como $E(Y)=E(Y, X-Y>0)+E(Y, X-Y \leq 0)$, temos que

$E(Y, X-Y \leq 0)=\left(\frac{2}{\pi}\right)^{\frac{1}{2}} \delta_{Y}-\left(\frac{\alpha\left(1-2 \omega^{2}\right)-\omega \beta}{\sqrt{2 \pi} \sqrt{1+\alpha^{2}\left(1-\omega^{2}\right)+(\alpha \omega+\beta)^{2}}}-\frac{\omega}{\sqrt{2 \pi}}\right)$ 
onde

$$
\begin{aligned}
& \delta_{Y}=\frac{\left(\alpha^{\prime} \omega^{\prime}+\beta^{\prime}\right) \sqrt{1-\omega^{\prime 2}}}{\sqrt{\left(\alpha^{\prime} \omega^{\prime}+\beta^{\prime}\right)^{2}+\left(1-\omega^{\prime 2}\right)+\left(\alpha^{\prime}+\omega^{\prime} \beta^{\prime}\right)^{2}-2 \omega^{\prime}\left(\alpha^{\prime}+\omega^{\prime} \beta^{\prime}\right)\left(\alpha^{\prime} \omega^{\prime}+\beta^{\prime}\right)}} \\
& =\frac{2 \omega\left[\alpha\left(1-2 \omega^{2}\right)-\omega \beta\right] \sqrt{1-\omega^{2}}}{\sqrt{\left[\alpha\left(1-2 \omega^{2}\right)-\omega \beta\right]^{2}+4 \omega^{2}\left(1-\omega^{2}\right)+(\alpha+\omega \beta)^{2}-2\left(1-2 \omega^{2}\right)(\alpha+\omega \beta)\left[\alpha\left(1-2 \omega^{2}\right)-\omega \beta\right]}}
\end{aligned}
$$

- $\quad$ Cálculo de $E\left(X^{2} \mid X-Y>0\right)$ e $E\left(X^{2} \mid X-Y \leq 0\right)$ :

$$
\begin{aligned}
E\left(X^{2}, X-Y>\right. & 0)=E\left(X^{2}, V>0\right) \\
& =\int_{0}^{\infty} \int_{-\infty}^{\infty} 2 x^{2} \phi_{\omega}(x, v) \Phi(\alpha x+\beta v) d x d v \\
& =2 \int_{0}^{\infty} \phi(v) \int_{-\infty}^{\infty} x^{2} \phi_{\omega}(x \mid v) \Phi(\alpha x+\beta v) d x d v \\
& =2 \int_{0}^{\infty} \phi(v) \int_{-\infty}^{\infty} x^{2} \frac{e^{\frac{-(x-\omega v)^{2}}{2\left(1-\omega^{2}\right)}}}{\sqrt{2 \pi} \sqrt{1-\omega^{2}}} \Phi(\alpha x+\beta v) d x d v \\
& =2 \int_{0}^{\infty} \phi(v) \int_{-\infty}^{\infty}\left(\sqrt{1-\omega^{2}} z+\omega v\right)^{2} \frac{e^{\frac{-z^{2}}{2}}}{\sqrt{2 \pi}} \Phi\left(\alpha \sqrt{1-\omega^{2}} z+(\alpha \omega+\beta) v\right) d z d v \\
& =\frac{\sqrt{1+\alpha^{2}\left(1-\omega^{2}\right)}}{\pi\left[1+\alpha^{2}\left(1-\omega^{2}\right)+(\alpha \omega+\beta)^{2}\right]}\left\{2 \alpha \omega\left(1-\omega^{2}\right)+\omega^{2}(\alpha \omega+\beta)\right. \\
& \left.-\frac{\alpha^{2}\left(1-\omega^{2}\right)^{2}(\alpha \omega+\beta)}{\left[1+\alpha^{2}\left(1-\omega^{2}\right)\right]}\right\}+1-2 \Psi \frac{-(\alpha \omega+\beta)}{\sqrt{1+\alpha^{2}\left(1-\omega^{2}\right)+(\alpha \omega+\beta)^{2}}}(0,0) .
\end{aligned}
$$

Como $E\left(X^{2}\right)=E\left(X^{2}, X-Y>0\right)+E\left(X^{2}, X-Y \leq 0\right)$, temos que

$$
\begin{aligned}
E\left(X^{2} \mid X-Y \leq\right. & 0) \\
& =\frac{-\sqrt{1+\alpha^{2}\left(1-\omega^{2}\right)}}{\pi\left[1+\alpha^{2}\left(1-\omega^{2}\right)+(\alpha \omega+\beta)^{2}\right]}\left\{2 \alpha \omega\left(1-\omega^{2}\right)+\omega^{2}(\alpha \omega+\beta)\right. \\
& \left.-\frac{\alpha^{2}\left(1-\omega^{2}\right)^{2}(\alpha \omega+\beta)}{\left[1+\alpha^{2}\left(1-\omega^{2}\right)\right]}\right\}+2 \Psi \frac{-(\alpha \omega+\beta)}{\frac{\sqrt{1+\alpha^{2}\left(1-\omega^{2}\right)+(\alpha \omega+\beta)^{2}}}{(0,0) .}}
\end{aligned}
$$


- $\quad$ Cálculo de $E\left(Y^{2} \mid X-Y>0\right)$ e $E\left(Y^{2} \mid X-Y \leq 0\right)$ :

$$
\begin{aligned}
E\left((X-Y)^{2}, X\right. & -Y>0)=4 \omega^{2} E\left(V^{2}, V>0\right) \\
& =4 \omega^{2} \int_{0}^{\infty} \int_{-\infty}^{\infty} 2 v^{2} \phi_{\omega}(x, v) \Phi(\alpha x+\beta v) d x d v \\
& =8 \omega^{2} \int_{0}^{\infty} v^{2} \phi(v) \int_{-\infty}^{\infty} \phi_{\omega}(x \mid v) \Phi(\alpha x+\beta v) d x d v \\
& =8 \omega^{2} \int_{0}^{\infty} v^{2} \phi(v) \int_{-\infty}^{\infty} \frac{e^{\frac{-(x-\omega v)^{2}}{2\left(1-\omega^{2}\right)}}}{\sqrt{2 \pi} \sqrt{1-\omega^{2}}} \Phi(\alpha x+\beta v) d x d v \\
& =8 \omega^{2} \int_{0}^{\infty} v^{2} \phi(v) \int_{-\infty}^{\infty} \frac{e^{\frac{-z^{2}}{2}}}{\sqrt{2 \pi}} \Phi\left(\alpha \sqrt{1-\omega^{2}} z+(\alpha \omega+\beta) v\right) d z d v \\
& =4 \omega^{2}\{1-2 \Psi \\
E(X(X-Y), X & \left.-Y>0 \frac{-(\alpha \omega+\beta)}{\sqrt{1+\alpha^{2}\left(1-\omega^{2}\right)+(\alpha \omega+\beta)^{2}}}(0,0)+\frac{(\alpha \omega+\beta) \sqrt{1+\alpha^{2}\left(1-\omega^{2}\right)}}{\pi\left[1+\alpha^{2}\left(1-\omega^{2}\right)+(\alpha \omega+\beta)^{2}\right]}\right\} \\
& =4 \omega \int_{0}^{\infty} \int_{-\infty}^{\infty} x v \phi_{\omega}(x, v) \Phi(\alpha x+\beta v) d x d v \\
& =4 \omega \int_{0}^{\infty} v \phi(v) \int_{-\infty}^{\infty} x \phi_{\omega}(x \mid v) \Phi(\alpha x+\beta v) d x d v \\
& =4 \omega \int_{0}^{\infty} v \int_{0}^{\infty} v \phi(v) \int_{-\infty}^{\infty} x \frac{e^{\frac{-(x-\omega v)^{2}}{2\left(1-\omega^{2}\right)}}}{\sqrt{2 \pi} \sqrt{1-\omega^{2}}} \Phi(\alpha x+\beta v) d x d v \\
& =41+\int_{-\infty}^{\infty}\left(\sqrt{1-\omega^{2}} z+\omega v\right) \frac{e^{\frac{-z^{2}}{2}}}{\sqrt{2 \pi}} \Phi\left(\alpha \sqrt{\left.1-\omega^{2} z+(\alpha \omega+\beta) v\right) d z d v}\right)
\end{aligned}
$$

Temos que

$$
\begin{aligned}
E\left(Y^{2}, X-Y>0\right) & \\
& =E\left(X^{2}, X-Y>0\right)-2 E(X(X-Y), X-Y>0)+E\left((X-Y)^{2}, X-Y>0\right) .
\end{aligned}
$$


Portanto

$$
\begin{aligned}
E\left(Y^{2}, X-Y>\right. & 0) \\
& =\frac{\sqrt{1+\alpha^{2}\left(1-\omega^{2}\right)}}{\pi\left[1+\alpha^{2}\left(1-\omega^{2}\right)+(\alpha \omega+\beta)^{2}\right]}\left\{2 \alpha \omega\left(1-\omega^{2}\right)+5 \omega^{2}(\alpha \omega+\beta)\right. \\
& \left.-\frac{\alpha^{2}\left(1-\omega^{2}\right)^{2}(\alpha \omega+\beta)}{\left[1+\alpha^{2}\left(1-\omega^{2}\right)\right]}-4 \omega(\alpha+\omega \beta)\right\}+1-2 \Psi \frac{-(\alpha \omega+\beta)}{\sqrt{1+\alpha^{2}\left(1-\omega^{2}\right)+(\alpha \omega+\beta)^{2}}}
\end{aligned}
$$

Como $E\left(Y^{2}\right)=E\left(Y^{2}, X-Y>0\right)+E\left(Y^{2}, X-Y \leq 0\right)$, temos que

$E\left(Y^{2} \mid X-Y \leq 0\right)$

$$
\begin{aligned}
& =\frac{-\sqrt{1+\alpha^{2}\left(1-\omega^{2}\right)}}{\pi\left[1+\alpha^{2}\left(1-\omega^{2}\right)+(\alpha \omega+\beta)^{2}\right]}\left\{2 \alpha \omega\left(1-\omega^{2}\right)+5 \omega^{2}(\alpha \omega+\beta)\right. \\
& \left.-\frac{\alpha^{2}\left(1-\omega^{2}\right)^{2}(\alpha \omega+\beta)}{\left[1+\alpha^{2}\left(1-\omega^{2}\right)\right]}-4 \omega(\alpha+\omega \beta)\right\}+2 \Psi \frac{-(\alpha \omega+\beta)}{\sqrt{1+\alpha^{2}\left(1-\omega^{2}\right)+(\alpha \omega+\beta)^{2}}}
\end{aligned}
$$

- $\quad$ Cálculo de $E(X Y \mid X-Y>0)$ e $E(X Y \mid X-Y \leq 0)$ :

Temos que $E(X Y, X-Y>0)=E\left(X^{2}, X-Y>0\right)-E(X(X-Y), X-Y>0)$.

Portanto

$$
\begin{aligned}
E(X Y, X-Y> & 0) \\
& =\frac{\sqrt{1+\alpha^{2}\left(1-\omega^{2}\right)}}{\pi\left[1+\alpha^{2}\left(1-\omega^{2}\right)+(\alpha \omega+\beta)^{2}\right]}\left\{2 \alpha \omega\left(1-\omega^{2}\right)+\omega^{2}(\alpha \omega+\beta)\right. \\
& \left.-\frac{\alpha^{2}\left(1-\omega^{2}\right)^{2}(\alpha \omega+\beta)}{\left[1+\alpha^{2}\left(1-\omega^{2}\right)\right]}-2 \omega(\alpha+\omega \beta)\right\} \\
& +\left(1-2 \omega^{2}\right)\left[1-2 \Psi \frac{-(\alpha \omega+\beta)}{\sqrt{1+\alpha^{2}\left(1-\omega^{2}\right)+(\alpha \omega+\beta)^{2}}}(0,0)\right] .
\end{aligned}
$$

Como $E(X Y)=E(X Y, X-Y>0)+E(X Y, X-Y \leq 0)$, temos que

$$
\begin{aligned}
E(X Y, X-Y \leq & 0) \\
& =\left(1-2 \omega^{2}\right)\left[2 \Psi \frac{-(\alpha \omega+\beta)}{\sqrt{1+\alpha^{2}\left(1-\omega^{2}\right)+(\alpha \omega+\beta)^{2}}}(0,0)\right] \\
& -\frac{\sqrt{1+\alpha^{2}\left(1-\omega^{2}\right)}}{\pi\left[1+\alpha^{2}\left(1-\omega^{2}\right)+(\alpha \omega+\beta)^{2}\right]}\left\{2 \alpha \omega\left(1-\omega^{2}\right)+\omega^{2}(\alpha \omega+\beta)\right. \\
& \left.-\frac{\alpha^{2}\left(1-\omega^{2}\right)^{2}(\alpha \omega+\beta)}{\left[1+\alpha^{2}\left(1-\omega^{2}\right)\right]}-2 \omega(\alpha+\omega \beta)\right\} .
\end{aligned}
$$




\section{Referências}

Alsina, C., Frank, M.J. and Schweizer, B. (2006). Associative Functions: Triangular Norms and Copulas. World Scientific.

Alvoni, E. and Papini, P. L. (2007). Quasi-concave copulas, asymmetry and transformations. Comment.Math.Univ.Carolin. 48, 311-319.

Anjos, U., Ferreira, F., Kolev, N. e Mendes, B. (2004). Modelando Dependências Via Cópulas. Minicurso $16^{\circ}$ SINAPE, $143 \mathrm{pg}$.

Azzalini, A. and Valle, A. D. (1996). The Multivariate Skew-Normal Distribution. Biometrika 83, 715-726.

Baets, B. D., Meyer, H. D. and Mesiar, R. Asymmetric Semilinear Copulas. Kybernetika 43, 221233.

Bairamov, I., Kotz, S. and Kozubowski, T. J. (2003). A New Measure of Linear Local Dependence. Statistics 37, 243-258.

Bell, C. B. and Haller, H. S. (1969). Bivariate symmetry tests: parametric and nonparametric. The Annals of Mathematical Statistics 40, 259-269.

Belzunce, F., Castaño, A., Olvera-Cervantes, A., Suárez-Llorens, A. (2007). Quantile curves and dependence structure for bivariate distributions. Computational Statistics \& Data Analysis 51, 5112-5129.

Capéraà, P., Fougères, A.-L. and Genest, C. (1997). A stochastic ordering based on a decomposition of Kendall's tau. In Distributions with given Marginals and Moment Problems, ed. By V. Beneš and J. Štěpán, 81-86.

Durante, F. and Papini, P.L. (2007). A weakening of Schur-concavity for copulas. Fuzzy Sets and Systems 158, 1378-1383.

Embrechts, P., Lindskog, F. and McNeil, A. (2003). Modelling Dependence with Copulas and Applications to Risk Management. Handbook of Heavy Tailed Distributions in Finance. Elsevier.

Embrechts, P., McNeil, A. and Strauman, D. (2002). Correlation and dependence in risk management: properties and pitfalls. In Risk Management: Value at Risk and Beyond, ed. by M. Dempster and H.K. Moffatt, 176-223.

Ernst, M. D. and Schucany, W. R. (1999). A class of permutation tests of bivariate interchangeability. Journal of the American Statistical Association 94, 273-284. 
Ferreira, F. H. (2003). Alguns Resultados Sobre Modelagem do Fenômeno de Dependência Através de Cópulas. Dissertação de Mestrado. Instituto de Matemática e Estatística Universidade de São Paulo.

Fredricks, G. A. and Nelsen, R. B. (2007). On the relationship between Spearman's rho and Kendal's tau for pairs of continuos random variables. Journal of Statistical Planning and Inference 137, 2143-2150.

Goodman L. (1969). How to ransack social mobility tables and other kinds of cross-classification tables. American Journal of Sociology 75, 1-40.

Holland P.W. and Wang Y.J. (1987). Dependence functions for continous bivariates densities. Communications in Statistics: Theory and Methods 16, 863-876.

Hollander, M. (1971). A nonparametric test for bivariate symmetry. Biometrika 58, 203-212.

Jones M. (1996). The local dependence function. Biometrika 83, 899-904.

Klement, E. P. and Mesiar, R. (2006). How non-symmetric can a copula be? Comment. Math. Univ. Carolinae 47, 141-148.

Klössner, S. (2007). Empirical evidence: intraday returns are neither symmetric nor Lévy processes. Versão preliminar.

Kolev, N. and Paiva, D. (2008). Random sums of exchangeable variables and actuarial applications. Insurance, Mathematics \& Economics 42, 147-153.

Kurowicka, D. and Cooke, R. (2006). Uncertainty Analysis with High Dimensional Dependence Modelling, Wiley.

Lien, D. and Balakrishnan, N. (2003). Conditional Correlation Analysis of order statistics from bivariate normal distribution with an application to evaluating inventory effects in futures market. Statistics \& Probability Letters 63, 249-257.

Ling, C.H. (1965). Representation of associative functions. Publ. Math. Debrecen 12, 189-212.

Mikusinski, P., H. Sherwood, e M. Taylor (1992). The Fréchet bounds revisited. Real Analysis Exchange 17, 759-764.

Nelsen, R. (2007). Extremes of Nonexchangeability. Statitical Papers 48, 329-336.

Nelsen, R. (2006). An Introduction to Copulas, $2^{\text {nd }}$ Ed. Springer, New York. 
Nelsen, R., Quesada-Molina, J., Rodrígues-Lallena, J. and Úbeda-Flores, M. (2003). Kendall Distribution functions. Statistics \& Probability Letters 65, 263-268.

Nelsen, R., Quesada-Molina, J., Rodrígues-Lallena, J. and Úbeda-Flores, M. (2001). Distribution function of copulas: a class of bivariate probability integral transforms. Statistics \& Probability Letters 54, 277-282.

Schmid, F. and Schmidt, R. (2007). Multivariate conditional versions of Spearman's rho and related measures of tail dependence. Journal of Multivariate Analysis 98, 1123-1140.

Snijders, T. (1981). Rank tests for bivariate symmetry. The Annals of Statistics 9, 1087-1095. Yanagimoto, T. and Sibuya, M. (1972). Stochastically larger component of a random vector. Ann. Inst. Statist. Math. 24, 259-269. 